\title{
Parallelization of Vector Fitting Algorithm for GPU Platforms
}

\author{
by \\ Naveen Kumar Elumalai, B. E., \\ A thesis submitted to the Faculty of Graduate Studies and Research \\ in partial fulfilment of the requirements for the degree of \\ Master of Applied Sciences \\ Carleton Institute of Electrical and Computer Engineering \\ Department of Electronics \\ Faculty of Engineering \\ Carleton University \\ Ottawa, Ontario, Canada
}

(c) Naveen Kumar Elumalai, 2019 
The undersigned recommend to the Faculty of Graduate Studies and Research acceptance of this thesis,

"Parallelization of Vector Fitting Algorithm for GPU Platforms"

submitted by Naveen Kumar Elumalai ( B.E.) in partial fulfillment of the requirements for the degree of Master of Applied Sciences

Thesis Supervisor

Professor Ram Achar

Chairman, Department of Electronics

Professor Niall Tait

Carleton University, May 2019 


\section{Abstract}

With the continually increasing operating frequencies and decreasing signal transition times, high-speed effects of interconnect structures are becoming increasingly influential in determining the performance of modern electronic designs. High-Speed effects can be many, such as delay, attenuation, cross talk and ground bounce, etc., which if not addressed properly can lead to failed designs. Hence accurate modeling and simulation of high-speed modules becomes a necessity in today's designs.

The modules of importance at higher frequencies span the diverse design hierarchy such as chip, package and system level designs. These can be multiconductor transmission lines, package pins or complex electromagnetic modules. At higher frequencies they are often characterized by electromagnetic tools yielding tabulated scattering parameter based multiport descriptions or characterized directly using multiport measurements. However, integrating such tabulated data models in regular SPICE like tool environment is a challenge. This is addressed in the literature by using direct least squares approximation to synthesize a rational function model, however, it often encountered the problem of ill-conditioning. This was handled by the Vector Fitting (VF) technique which has gained popularity in the recent years, not only in electronic designs but also in other areas where system identification using multiport data is warranted. However, VF technique suffers in the presence of large number of ports or poles and becomes compu- 
tationally slower. To address this problem, recently, parallel vector fitting using multicore CPU environment was proposed in the literature.

In this thesis, vector fitting algorithm is advanced by proposing the use of the emerging computing platform of GPUs. Several parallel strategies are explored for optimal use of resources: CPUs, GPU and memory, for arriving at better computational performance. Numerical examples are presented which demonstrate the viability of using GPUs based on the size of the problem under consideration. An optimally designed algorithm for the combined GPU and CPU platforms can yield better performance compared to the case of using just the CPUs. 


\section{Acknowledgements}

I would like to thank my thesis advisor Professor Ram Achar of the Electronics Department at Carleton University. I would also like to thank Professor Wai Kong Lee at University of Tunku, who was always there to help when I ran into issues with GPU. They steered me in the right direction whenever I needed it. I am gratefully indebted to their very valuable guidance on this thesis.

I would also like to thank Srinidhi Ganeshan with whom I could have research discussions, who was a great source of confidence, encouragement and support.

I would also like to show my gratitude to my father Elumalai, my mother Devi, my sister Jayashree for being supportive and for the continuous encouragement throughout my years of study. 


\section{Table of Contents}

1 Introduction $\quad \mathbf{1 0}$

1.1 Background and Motivation . . . . . . . . . . . . 10

1.2 Organization of the Thesis ............... 15

2 Overview of Vector Fitting 17

2.1 Brief Overview . . . . . . . . . . . . . 17

2.2 Vector Fitting by Pole Relocation . . . . . . . . . . . . 17

2.2.1 Pole Calculation . . . . . . . . . . . . . . . . 18

2.2.2 Evaluation of Poles of $f(s) \ldots \ldots 23$

2.2.3 Selection of Initial set of poles . . . . . . . . . . 26

2.3 A Computational Complexity Analysis of Vector Fitting method . 26

2.4 Challenges associated with Pole Computing for Multiport Tabulated Subnetworks . . . . . . . . . . . . . . . . . 27

3 Overview of Parallel Vector Fitting 28

3.1 Overview of formulation of multiport VF suitable for Parallel com-

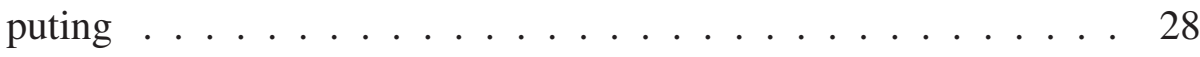

3.2 Splitting Strategies . . . . . . . . . . . . . 34 
3.2.1 None Splitting Strategy . . . . . . . . . . . . . . . . . 34

3.2.2 All Splitting Strategy . . . . . . . . . . . . . . . . 40

3.2.3 Column Splitting . . . . . . . . . . . . . . . . . . . 44

4 Review of GPU Architectures and Programming 49

4.1 Introduction . . . . . . . . . . . . . . . . . . . . . . . . . . 49

4.2 GPU Architecture . . . . . . . . . . . . . . . . . . . . 49

4.2 .1 Terminology . . . . . . . . . . . . . 50

4.2 .2 Memory Hierarchy . . . . . . . . . . . . . . . . 51

4.3 CUDA Programming Model . . . . . . . . . . . . 55

4.3 .1 cudaMalloc . . . . . . . . . . . . . . . 55

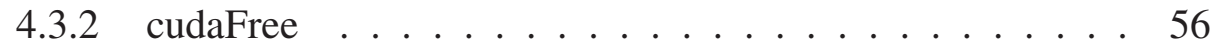

$4.3 .3 \quad$ Kernel Call . . . . . . . . . . . . . . . . . 56

4.3.4 Data transfer between host memory and device memory - 56

5 GPU based Parallel VF 58

5.1 Approach-1 : Formulation of $\boldsymbol{A}_{i j}$ in CPU followed by immediate QR factorization and residue evaluation in GPU for None Splitting Strategy . . . . . . . . . . . . . . . . . . . . 59

5.1.1 Approach-1 with multiple CPUs and a single GPU . . . 59

5.1.2 Discussion on CPU to GPU, GPU to CPU Data transfer time for Approach-1 . . . . . . . . . . . . . . . . 62

5.1.3 Discussion on collection time for $\boldsymbol{R}_{l}^{22}$ matrices for residue vector $(c)$ evaluation for Approach-1 . . . . . . . . . . 66

5.1.4 Discussion on QR factorization time for Approach-1 _ . 66 


\subsubsection{Performance comparison of Approach-1 with multiple CPUs}

and a single GPU versus just the multiple CPUs . . . . . 69

5.1.6 Approach-1 with Multiple CPUs and two GPUs . . . . . . 70

5.1.7 Performance of Approach-1 with 2-GPUs and multiple CPUs 71

5.2 Approach-2: Transfer of block of $\boldsymbol{A}_{l}$ matrices from CPU to GPU and parallel QR factorization in CPUs and GPU (single scaling

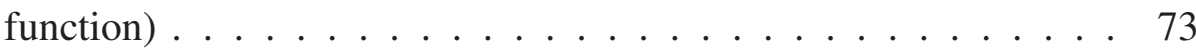

5.2.1 Performance Comparison of Approach-2 (with multiple CPUs and one GPU) and just the multiple CPUs . . . . . . 78

5.3 Approach-3: Transfer of block of $\boldsymbol{A}_{l}$ matrices from CPUs to GPU, parallel QR factorization and residue evaluation in CPUs as well as in GPU (individual scaling functions) . . . . . . . . . . . . 80

5.3.1 Performance comparison of Approach-3 (with multiple CPUs and one GPU) and just the multiple CPUs (All Splitting) . 85

$\begin{array}{llr}6 & \text { Computational Results } & 87\end{array}$

6.1 Example 1:30 port network .............. 88

6.2 Example $2: 60$ port network . . . . . . . . . . . . 92

6.3 Example $3: 120$ port network . . . . . . . . . . 96

$\begin{array}{lll}7 & \text { Conclusion } & 100\end{array}$

$\begin{array}{lr}\text { References } & 101\end{array}$ 


\section{List of figures}

4.1 CUDA Programming Model: Grid, Block, Warp, Thread Architecture .......................... 50

4.2 Cuda Memory Architecture . . . . . . . . . . . 52

5.2 Transfer Time for Approach-1 for multiport VF . . . . . . . 63

5.3 Comparison of time for QR factorization for one $\boldsymbol{A}_{l}$ element in GPU and CPU . . . . . . . . . . . . . . 67

5.4 Comparison of Approach-1 with multiple CPUs and a single GPU versus just the CPUs . . . . . . . . . . . . . . 69

5.5 Performance comparison of Approach-1 with multiple CPUs and a single GPU, Approach-1 with multiple CPUs and two GPUs with CPUs alone. . . . . . . . . . . . . . . 71

5.7 Performance Comparison of Approach-2 and just the Multiple

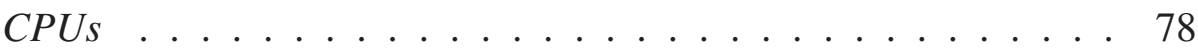

5.9 Performance Comparison of Approach-3 and just the Multiple CPUs for the All Splitting Strategy . . . . . . . . . . . . . 85 
6.1 Performance Comparison of Approach-1 with multiple CPUs and a single GPU, Approach-2 and Approach-3 versus the case of just the Multiple CPUs . . . . . . . . . . . . . . . . . 88

6.2 Comparison of sample frequency responses for Example 1 . . . 90

6.3 Comparison of sample frequency responses for Example 1 . . . 91

6.4 Performance Comparison of Approach-1 with multiple CPUs and a single GPU, Approach-2 and Approach-3 versus the case of just the Multiple CPUs . . . . . . . . . . . . . . . . . . . 92

6.5 Comparison of sample frequency responses for Example 2 . . . . 94

6.6 Comparison of sample frequency responses for Example 2 . . . 95

6.7 Performance Comparison of Approach-1 with multiple CPUs and a single GPU, Approach-2 and Approach-3 versus the case of just the Multiple CPUs . . . . . . . . . . . . . . . . . . . 96

6.8 Comparison of sample frequency responses for Example 3 . . . 98

6.9 Comparison of sample frequency responses for Example 3 . . . . 99 


\section{List of Tables}

5.1 Data transfer time for Approach-1 for multiport VF . . . . . . 65

$5.2 n_{g}$ and $n_{c}$ values for Approach-2 for multiport VF . . . . . . 80

$6.1 n_{g}$ and $n_{c}$ values for Example 1 for multiport VF . . . . . . . 89

$6.2 n_{g}$ and $n_{c}$ values for Example 3 for multiport VF . . . . . . . 97 


\section{Chapter 1}

\section{Introduction}

\subsection{Background and Motivation}

The recent trend in VLSI for high frequency and lower power presents significant challenges for design and CAD techniques at both the chip and package levels. This is because, the operating frequencies are fast reaching the vicinity of several gigahertz range and switching times are getting to sub-nanosecond levels. This has placed higher demands on interconnect performance and has also highlighted the previously negligible effects of interconnects such as signal delay, reflections and cross talk. In today's designs, these interconnects are responsible for most of the signal degradation in high-speed systems.

The problems in high-speed interconnects need to be anticipated at the early design stage. If not predicted, these interconnect effects can cause logical glitches that render a fabricated circuit inoperable. Alternatively, these interconnects can also distort analog signals such that they fail to meet the specifications. The accurate prediction of interconnect effects is necessary since extra iterations in the design cycle are expensive. Therefore, it is important for designers to simulate the entire design along with interconnect sub-circuits in an efficient way. 
Recently, the modelling and the characterization of the tabulated data representing the interconnect behaviour is becoming an integral part of signal integrity validations, due to the complex nature of high-speed modules [1] - [3]. The tabulated data can be in various forms, such as, admittance (Y), impedance (Z), scattering (S), hybrid (T) parameters (henceforth referred to tabulated data in this thesis). This tabulated data can be obtained from measurements or full wave simulation. Important applications of such measurements include high-speed interconnects, packages, vias, connectors, non-uniform transmission lines and on-chip passive components, such as inductors and transformers. However, transient simulation of tabulated data is a CPU expensive process due to large bandwidth requirements and mixed frequency/time problem.

This is because, the interconnect circuits are terminated by nonlinear elements such as drivers and receivers, which are characterized in time domain in terms of ordinary differential equations (ODEs). However, tabulated data are best described in the frequency-domain and do not have a direct time domain representation compatible with ODEs. Simulation of such a mixed frequency/time domain formulation cannot be easily handled by traditional solvers such as SPICE [4].

In the literature, various approaches can be found to address the mixed frequency/ time problem concerning tabulated data. These approaches can be classified into two categories. First, the transient simulation is performed based on the traditional convolution process [1], [3], where the frequency-dependent sampled data is first converted to time domain using inverse Fourier transform (IFFT). Here, special attention is needed to avoid aliasing. Low pass filtering and extrapolation of the frequency-domain data are needed to reduce the time domain ripples associated 
with the IFFT. After IFFT is performed, the time-domain data is convoluted with the transient responses of both the nonlinear load and input excitation. However, this method becomes computationally infeasible for large multiport systems.

The second approach is based on obtaining a reduced-order model [5] - [8] for the frequency-dependant sampled data and performing transient analysis using recursive convolution [9] - [10] or the macromodelling. In the macromodelling approach, the frequency-domain rational-function model is converted to a set of ordinary differential equations [5], [11]. There are several challenges associated with this, such as, no guarantee of preserving the passivity in the resulting macromodel while maintaining accuracy.

Passivity implies that a network cannot generate more energy than it absorbs. The loss of passivity can be a serious problem because transient simulation of a non-passive macromodel may encounter artificial oscillations in the presence of other terminations. The macromodel that are only stable but not passive, can produce unstable networks when connected to other passive loads. At the same time, a passive macromodel when terminated with any arbitrary passive load always guarantees the stability of the overall resulting network. Therefore, in addition to macromodel stability, macromodel passivity is required to guarantee the stability of the overall circuit. Ensuring passivity involves passivity verification and compensation. This can be accomplished using first order perturbation based iterative algorithms such as [12] - [13].

Modelling of tabulated data was also handled via system identification methods such as direct least squares approximation to synthesize a rational function model, however, it often encountered the problem of ill-conditioning. This was handled 
by the Vector Fitting (VF) technique which has gained popularity in the recent years, not only in the electronic design but also in other areas where system identification using multiport data is warranted.

In the Vector Fitting method, instead of directly extracting the poles of the system by fitting the given data to a rational function based formulation (which tends to be ill-conditioned for broadband data and for large number of poles), it assumes an initial set of poles and a scaling function. Using this initial information, Sanathanan Koerner (SK) iterations [10] are reformulated using a partial fraction basis (wherein the initial assumed set of poles represent the poles of the partial fraction). Using the resulting set of equations, residues of the scaling functions are evaluated, which in turn are used to calculate the zeros of the scaling function, which represents the relocated poles of the partial fraction. These relocated poles are used as the new set of poles for the successive SK iterations until the poles converge. The robustness of this method is mainly due to the use of rational basis instead of polynomials which tends to be numerically superior when initial poles are carefully chosen. However, VF technique suffers in the presence of large number of ports or poles and becomes computationally slower.

To improve the efficiency of VF, a computationally efficient version was proposed [25]. In this approach, instead of doing the $Q R$ factorization of the entire matrix, it was shown that $\mathrm{QR}$ factorization of only blocks of the matrix corresponding to the individual S-elements are needed, which reduces the computational cost significantly.

Also to improve the performance of VF, recently parallelization of the vector fitting algorithm using the multi-CPU environment can be found in the literature 
[16]. The main approaches adopted in this were:

(a) All Splitting Strategy, in which all steps of the VF (formulation, QR factorization and residues of the scaling function) are done in parallel in the available CPUs (the method assumed individual scaling functions for individual S-elements). The method yielded individual pole sets for each of the S-elements.

(b) None Splitting Strategy, in which all VF steps were done in parallel in the available CPUs (formulation of matrices corresponding to each S-elements and its QR factorization) except for the last step of the iteration, to compute the residues of the scaling function, which was done in a single CPU (the method used only one scaling function for the entire matrix). The method yielded a common pole set for the entire multiport S-elements.

In this thesis, vector fitting algorithm is advanced by proposing the use of the emerging computing platform of GPUs. Several parallel strategies are explored for optimal use of resources: CPUs, GPU and memory, for arriving at better computational performance. Specifically, the contributions of the thesis are as follows

1. An algorithm, Approach-1, is developed for adoption of the None Splitting Strategy which was developed in [16] for just the multiple CPU environment, to the mixed CPU and GPU platform. In this algorithm, QR factorization of the matrices corresponding to individual S-parameter matrix elements is done sequentially in GPU. All other vector fitting tasks are handled by the CPU. 
2. An algorithm, Approach-2, is developed for adoption of the None Splitting Strategy to the mixed CPU and GPU environment, wherein matrices for QR factorization corresponding to a block of S-matrix elements are handled parallely in GPU, while the remaining elements are processed simultaneously and parallely in the available CPUs.

3. An algorithm, Approach-3, is developed for adoption of the All Splitting Strategy which was developed in [16] for just the CPUs, to the mixed CPU and GPU environment. Here, GPU not only does the parallel QR factorization of matrices corresponding to a block of the S-matrix elements, it also follows it up by evaluating the residues of the individual scaling functions. The remaining S-elements are simultaneously and parallely processed by the available CPUs.

4. This thesis also explores the impact of the various data transfer times between the CPUs and GPU depending on the approach used.

Numerical examples are presented which demonstrate the viability of using GPUs based on the size of the problem under consideration. An optimally designed algorithm using the combined GPU and CPU environment can yield better performance compared the case of using just the CPUs.

Rest of the thesis is organized as follows.

\subsection{Organization of the Thesis}

The thesis is organized as follows. In Chapter 2, an overview of system identification using vector fitting algorithm from a given set of tabulated data is pre- 
sented. Chapter 3 presents an overview of parallelization algorithms for vector fitting. Chapter 4 presents a brief overview of GPU architecture and programming. Chapter 5, presents the development of the proposed GPU based vector fitting algorithms. Numerical results are presented in Chapter 6, while conclusions are presented in Chapter 7. 


\section{Chapter 2}

\section{Overview of Vector Fitting}

\subsection{Brief Overview}

Vector fitting is a widely used algorithm for system identification from the given tabulated data which employs principles outlined in [14]. Typically, high-speed modules are represented using multiport parameters in the form of table of frequency samples over the desired bandwidth. This data-set is used as input to the vector fitting algorithm which synthesizes rational-function-based model. Vector fitting is numerically robust method for rational approximation in the frequencydomain and it has evolved as well as matured during the last two decades.

\subsection{Vector Fitting by Pole Relocation}

Consider the rational-function in the form of pole residue formulation, as follows

$$
f(s)=\sum_{i=1}^{n} \frac{\hat{c}_{i}}{s-\overline{p_{i}}}+d+s e
$$

here the residues $\left(\hat{c}_{i}\right)$ and poles $\left(\overline{p_{i}}\right)$ can be real or complex conjugate pairs. $n$ is the number of poles and residues, $d$ and $e$ are real. The problem here is to estimate all the coefficients in (2.2) so that least-square approximation of $f(s)$ is obtained 
over the given frequency interval. The nonlinear optimization problem in terms of unknown poles $\overline{p_{i}}$ appearing in denominator is solved as linear problem in two steps as follows.

\subsubsection{Pole Calculation}

The first step involves doing an iterative procedure that refines an initial estimate of poles $\overline{p_{i}}$. The scaling function and the starting poles of the following form is considered below

$$
\sigma(s)=\sum_{i=1}^{n} \frac{c_{i}}{s-\overline{p_{i}}}+1
$$

Multiplying (2.1) with (2.2) (i.e., multiplying the scaling function, $\sigma(s)$ with $f(s)$ ), we get a scaled function

$$
\sigma(s) f(s)=\sum_{i=1}^{n} \frac{\tilde{c}_{i}}{s-\overline{p_{i}}}+d+s e
$$

The scaled function is represented as $F(s)$

$$
F(s)=\sum_{i=1}^{n} \frac{\tilde{c}_{i}}{s-\overline{p_{i}}}+d+s e
$$

Substituting (2.2) in (2.3) we get

$$
\left[\sum_{i=1}^{n} \frac{c_{i}}{s-\overline{p_{i}}}+1\right] f(s)=\sum_{i=1}^{n} \frac{\tilde{c}_{i}}{s-\overline{p_{i}}}+d+s e
$$


Here, $\overline{p_{i}}$ is the initial guess of poles and the number of unknowns is $n_{t}=2 n+2$

$$
\left[\sum_{i=1}^{n} \frac{c_{i}}{s-\overline{p_{i}}} f(s)+f(s)\right]=\sum_{i=1}^{n} \frac{\tilde{c}_{i}}{s-\overline{p_{i}}}+d+s e
$$

Rearranging (2.6)

$$
f(s)=\sum_{i=1}^{n} \frac{\tilde{c}_{i}}{s-\overline{p_{i}}}+d+s e-\left[\sum_{i=1}^{n} \frac{c_{i}}{s-\overline{p_{i}}} f(s)\right]
$$

Expanding (2.7)

$$
\left[\frac{\tilde{c}_{1}}{s-\overline{p_{1}}}+\ldots+\frac{\tilde{c}_{n}}{s-\overline{p_{n}}}+d+s e\right]-\left[\frac{c_{1} f\left(s_{k}\right)}{s-\overline{p_{1}}}+\ldots+\frac{c_{n} f\left(s_{k}\right)}{s-\overline{p_{n}}}\right]=f(s)
$$

Writing (2.7) for $k^{\text {th }}$ frequency point, we have

$$
\left[\frac{\tilde{c}_{1}}{s_{k}-\overline{p_{1}}}+\ldots+\frac{\tilde{c}_{n}}{s_{k}-\overline{p_{n}}}+d+s_{k} e\right]-\left[\frac{c_{1} f\left(s_{k}\right)}{s_{k}-\overline{p_{1}}}+\ldots+\frac{c_{n} f\left(s_{k}\right)}{s_{k}-\overline{p_{n}}}\right]=f\left(s_{k}\right)
$$

Next, expressing (2.9) for several frequency sample data $f\left(s_{k}\right)$ points, where $s_{k}$ $=j \omega$, we have,

$$
\begin{aligned}
& {\left[\frac{\tilde{c}_{1}}{s_{1}-\overline{p_{1}}}+\ldots+\frac{\tilde{c}_{n}}{s_{1}-\overline{p_{n}}}+d+s_{1} e\right]-\left[\frac{c_{1} f\left(s_{1}\right)}{s_{1}-\overline{p_{1}}}+\ldots+\frac{c_{n} f\left(s_{1}\right)}{s_{1}-\overline{p_{n}}}\right]=f\left(s_{1}\right)} \\
& {\left[\frac{\tilde{c}_{1}}{s_{2}-\overline{p_{1}}}+\ldots+\frac{\tilde{c}_{n}}{s_{2}-\overline{p_{n}}}+d+s_{2} e\right]-\left[\frac{c_{1} f\left(s_{2}\right)}{s_{2}-\overline{p_{1}}}+\ldots+\frac{c_{n} f\left(s_{2}\right)}{s_{2}-\overline{p_{n}}}\right]=f\left(s_{2}\right)}
\end{aligned}
$$




$$
\left[\frac{\tilde{c}_{1}}{s_{k}-\overline{p_{1}}}+\ldots+\frac{\tilde{c}_{n}}{s_{k}-\overline{p_{n}}}+d+s_{k} e\right]-\left[\frac{c_{1} f\left(s_{k}\right)}{s_{k}-\overline{p_{1}}}+\ldots+\frac{c_{n} f\left(s_{k}\right)}{s_{k}-\overline{p_{n}}}\right]=f\left(s_{k}\right)
$$

This leads to a set of algebraic equations of the form,

$$
A X=b
$$

where $\boldsymbol{b}=\left[f\left(s_{1}\right) f\left(s_{2}\right) f\left(s_{3}\right) \ldots f\left(s_{k}\right)\right]^{T}$

\section{Case1: Real Poles}

In case of real poles, writing (2.10) at several frequency points results in the following set of matrix equations

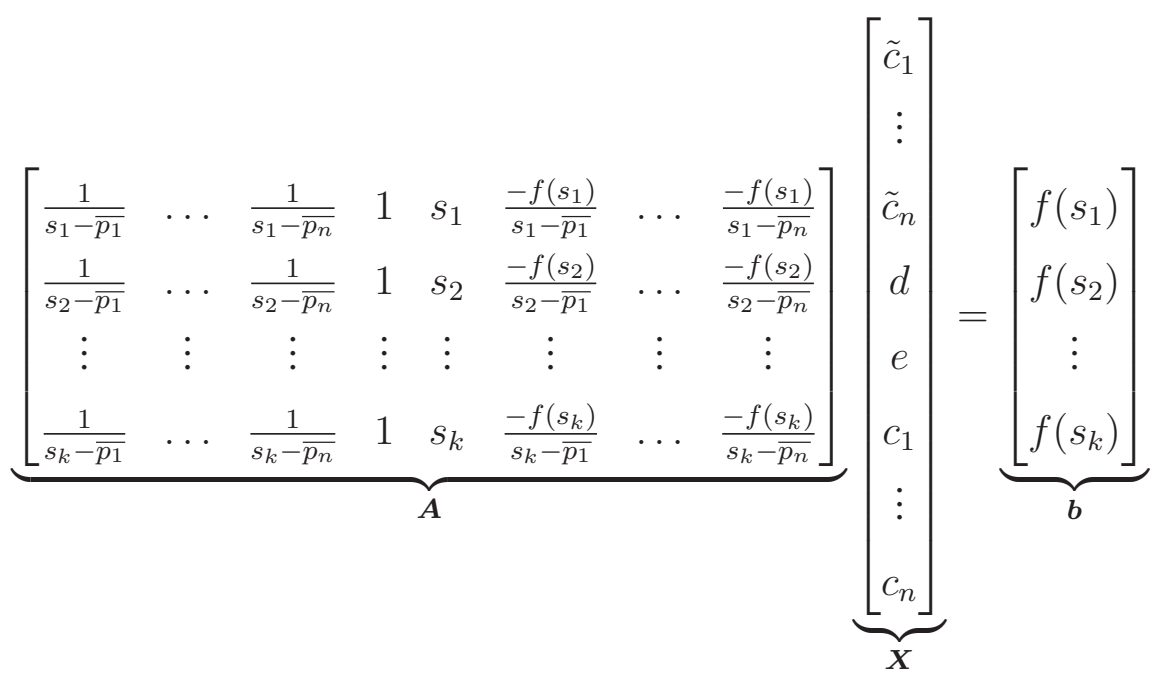

For real poles, the unknowns in vector $\boldsymbol{X}$ are real. 


\section{Case2: Complex Poles}

Complex poles exist in complex conjugate pairs. As a result, residues are also in complex conjugate pairs. Consider a complex pole along with its conjugate in

$$
\overline{p_{i}}=R e\left(p_{i}\right)+j \operatorname{Im}\left(p_{i}\right) ;{\overline{p_{i}}}^{*}=\operatorname{Re}\left(p_{i}\right)+j \operatorname{Im}\left(p_{i}\right)
$$

where $\bar{p}_{i}$ is the complex conjugate pair and $*$ is the complex conjugate operator. Therefore, residues of the scaled function $F(s)$ is represented by

$$
\tilde{c}_{i}=\operatorname{Re}\left(\tilde{c}_{i}\right)+j \operatorname{Im}\left(\tilde{c}_{i}\right) ; \tilde{c}_{i} *=\operatorname{Re}\left(\tilde{c}_{i}\right)-j \operatorname{Im}\left(\tilde{c}_{i}\right)
$$

and that of the scaling function by

$$
c_{i}=\operatorname{Re}\left(c_{i}\right)+j \operatorname{Im}\left(c_{i}\right) ; c_{i} *=\operatorname{Re}\left(c_{i}\right)-j \operatorname{Im}\left(c_{i}\right)
$$

For the above representation for the pole pair, (2.7) becomes

$$
f\left(s_{k}\right)=\frac{\tilde{c}_{1}}{s_{k}-\overline{p_{1}}}+\frac{\tilde{c}_{1}^{*}}{s_{k}-{\overline{p_{1}}}^{*}}+d+s_{k} e-f\left(s_{k}\right) \frac{c_{1}}{s_{k}-\overline{p_{1}}}-f\left(s_{k}\right) \frac{c_{1}^{*}}{s_{k}-{\overline{p_{1}}}^{*}}
$$

Substituting (2.14), (2.15) in (2.16), we get

$$
\begin{gathered}
{\left[\frac{\tilde{c}_{1}}{s_{k}-\overline{p_{1}}}+\frac{\tilde{c}_{2}}{s_{k}-\overline{p_{2}}}+\frac{\tilde{c}_{2}}{s_{k}-\bar{p}_{2}^{*}}+\frac{\tilde{c}_{2}^{*}}{s_{k}-\overline{p_{2}}}-\frac{\tilde{c}_{2}^{*}}{s_{k}-\bar{p}_{2}}{ }^{*}+d+s_{k} e\right]-} \\
{\left[\frac{c_{1}}{s_{k}-\overline{p_{1}}}+\frac{c_{2}}{s_{k}-\overline{p_{2}}}+\frac{c_{2}}{s_{k}-\overline{p_{2}}{ }^{*}}+\frac{c_{2}{ }^{*}}{s_{k}-\overline{p_{2}}}-\frac{c_{2}{ }^{*}}{s_{k}-\bar{p}_{2}}\right]=f\left(s_{k}\right)}
\end{gathered}
$$

Next, expressing (2.17) for $k$ different frequency points, resulting equations can 
be written in the form $\boldsymbol{A X}=\boldsymbol{b}$; here both $\boldsymbol{A}$ and $\boldsymbol{b}$ are split into real and imaginary parts as

$$
\left[\left(\begin{array}{c}
\operatorname{Re}(\boldsymbol{A}) \\
\operatorname{Im}(\boldsymbol{A})
\end{array}\right) \boldsymbol{X}=\left(\begin{array}{c}
\operatorname{Re}(\boldsymbol{b}) \\
\operatorname{Im}(\boldsymbol{b})
\end{array}\right)\right]
$$

The above equation (2.18) is solved using QR factorization technique. Equation (2.18) can be denoted as

$$
\widehat{A} \boldsymbol{X}=\widehat{b}
$$

Writing $\widehat{A}$ in terms of $\boldsymbol{Q}$ and $\boldsymbol{R}$ factors, where $\boldsymbol{Q}$ is the orthogonal matrix and $\boldsymbol{R}$ is upper triangular matrix

$$
Q R X=\widehat{b}
$$

To evaluate $\boldsymbol{X}$,

$$
\boldsymbol{R} \boldsymbol{X}=\boldsymbol{Q}^{T} \widehat{\boldsymbol{b}}
$$

or substituting $\boldsymbol{Q}^{T} \widehat{\boldsymbol{b}}=\widehat{\boldsymbol{b}}$, the following equation is solved to evaluate $\boldsymbol{X}$

$$
R X=\widehat{b}
$$

or

$$
\boldsymbol{X}=\boldsymbol{R}^{-1} \widehat{\boldsymbol{b}}
$$

Next, a rational-function approximation for the function $f(s)$ can be obtained by writing it in the fractional form. For this purpose, we first express the scaling function in (2.2) and scaled function in (2.3), as

$$
\sigma(s)=\frac{\prod_{i=1}^{n}\left(s-z_{i}\right)}{\prod_{i=1}^{n}\left(s-\overline{p_{i}}\right)}
$$


and

$$
\sigma f(s)=h \frac{\prod_{i=1}^{n}\left(s-z_{i}\right)}{\prod_{i=1}^{n}\left(s-\overline{p_{i}}\right)}
$$

Also from (2.5), it is evident that

$$
f(s)=\frac{\sigma f(s)}{\sigma(s)}
$$

From (2.26) and (2.24), it is clear that zeros of $\sigma(s)$ are poles of $f(s)$. Note that initial poles cancel in the division process as we started with the same initial poles for $\sigma(s)$ and $\sigma f(s)$. Thus, by calculating zeros of $\sigma(s)$, we get a better set of poles than the initial set of poles $\overline{p_{i}}$, for fitting the original function $f(s)$.

\subsubsection{Evaluation of Poles of $f(s)$}

Once the unknowns $c_{1}, \ldots, c_{n}$ are obtained by solving (2.23), zeros of $\sigma(s)$ can be calculated, depending on whether the initial guess for the poles is real or complex. In both these cases, zeros of $\sigma(s)$ corresponding to the eigenvalues of matrix $\boldsymbol{H}$, where $\boldsymbol{H}$ is given by,

$$
H=\psi-\mathbf{g} \phi^{T}
$$

Here the entities $\boldsymbol{\psi}, \boldsymbol{g}$ and $\phi$ can be expressed for real poles case and complex poles case as follows. 


\section{Case 1: Real Poles}

In case of real poles, $\boldsymbol{H}$ matrix of (2.27) can be written as,

$$
\boldsymbol{\psi}=\left[\begin{array}{ccc}
\bar{p}_{1} & & \\
& \ddots & \\
& & \bar{p}_{n}
\end{array}\right]_{n \times n}
$$

$$
\boldsymbol{g}=\left[\begin{array}{c}
1 \\
1 \\
\vdots \\
1
\end{array}\right]_{n \times 1}
$$

$$
\boldsymbol{\phi}=\left[\begin{array}{c}
c_{1} \\
c_{2} \\
\vdots \\
c_{n}
\end{array}\right]_{n \times 1}
$$

where $\psi$ is the diagonal matrix of the starting poles, $\boldsymbol{g}$ is a unit column vector, $\phi$ is the column vector of residues of $\sigma(s)$, corresponds to the starting poles computed in (2.23) of size $1 \times n$, where $n$ is the number of poles. 


\section{Case 2: Complex Poles}

In case of complex poles, $\boldsymbol{\psi}$ is defined as follows,

$$
\psi=\left[\begin{array}{lll}
\psi_{1} & & \\
& \ddots & \\
& & \psi_{n}
\end{array}\right]_{n \times n}
$$

where $\psi_{i}$ is represented by

$$
\begin{gathered}
\boldsymbol{\psi}_{\boldsymbol{i}}=\left(\begin{array}{cc}
\operatorname{Re}\left(\bar{p}_{i}\right) & \operatorname{Im}\left(\bar{p}_{i}\right) \\
-\operatorname{Im}\left(\bar{p}_{i}\right) & \operatorname{Re}\left(\bar{p}_{i}\right)
\end{array}\right) \\
\boldsymbol{\phi}=\left(\begin{array}{c}
\operatorname{Re}\left(c_{1}\right) \\
\operatorname{Im}\left(c_{1}\right) \\
\vdots \\
\operatorname{Re}\left(c_{n}\right) \\
\operatorname{Im}\left(c_{n}\right)
\end{array}\right)_{2 n \times 1} \\
\boldsymbol{g}=\left[\begin{array}{c}
2 \\
0 \\
\vdots \\
2 \\
0
\end{array}\right]_{2 n \times 1}
\end{gathered}
$$

Once the zeros of $\sigma(s)$ are calculated, they are used as new set of starting poles in the next iteration while solving (2.6). The next iteration provides a new set of zeros of $\sigma(s)$ which is again used in its next iteration as starting poles. The 
iterative process is continued until the poles converge.

It is to be noted that during the iterative process of vector fitting, some of the calculated poles may become unstable (i.e., the real part of some of the poles can become positive). This problem is overcome by inverting the sign of the real part of the pole [6] and re-executing the VF iterations.

\subsubsection{Selection of Initial set of poles}

Successful application of vector fitting requires judicious selection of initial set of poles. The following guidelines are used while selecting the initial set of poles [14]. For functions with distinct resonance peaks, starting poles are selected in complex conjugate pairs with the imaginary parts $\beta_{1}, \beta_{2}, \ldots, \beta_{n}$, linearly dis-

tributed over the frequency range of interest $\left[\omega_{\min }\right.$ to $\left.\omega_{\max }\right]$. Each pole-pair is selected as follows,

$$
p=-\alpha \pm j \beta
$$

with

$$
\alpha=\frac{\beta}{100}
$$

For smooth responses, real poles are selected linearly or logarithmically spaced over the frequency of interest.

\subsection{A Computational Complexity Analysis of Vector Fitting method}

The major computational cost associated with the VF process [16] is with regards to the $\mathrm{QR}$ factorization in each iteration. The number of floating-point operations (flops) for a QR factorization of matrix $\boldsymbol{A}$ of size $m$ by $n$ for a single element $\boldsymbol{S}_{i j}$ 
involved in VF algorithm [15], [16] is given below,

$$
F L_{Q R}(m, n)= \begin{cases}2 m^{2} n-\frac{2}{3} m^{3} & \text { if } n \geq m \\ 2 n^{2} m-\frac{2}{3} n^{3} & \text { if } n>m\end{cases}
$$

The computational cost to solve $(2.20)$ is $\operatorname{FL}_{Q R}(m, n)$ and the cost to solve, the right hand side of (2.21) is $2 m n p$ flops, where $Q$ matrix has the size $m \times n$ and $\widehat{\boldsymbol{b}}$ has the size $n \times p$. Equation (2.22) requires $n_{p}^{2}$ flops, where $\boldsymbol{R}$ matrix is of the size $n \times n$.

\subsection{Challenges associated with Pole Computing for Multiport Tabulated Sub- networks}

Consider a M-port measured network and its admittance matrix

$$
\boldsymbol{Y}(s)=\left[\begin{array}{ccc}
Y_{11} & Y_{12} & \ldots \\
\vdots & \ddots & \\
Y_{M 1} & & Y_{M M}
\end{array}\right]
$$

Conventionally, each element of the above admittance matrix is approximated by a rational-function. To find a common pole-set, the poles corresponding to individual elements of (2.38) are collected together. However, this approach can lead to large number of redundant poles in the macromodel resulting in an inefficient transient simulation. In addition, identifying a common multi-port pole set from pole-sets of individual elements of the admittance matrix is generally heuristic and can lead to errors. This is one the major challenges faced by the Vector Fitting, when the number of ports increases then VF becomes CPU expensive. 


\section{Chapter 3}

\section{Overview of Parallel Vector Fitting}

In Chapter 2, a brief review of the vector fitting method for high-order rationalfunction approximation of multiport tabulated data was presented. One of the challenges associated with the vector fitting (VF) is its relatively poor scalability with the increasing number of ports. When the number of input-output ports $(P)$ of the structure becomes large, excessive computational requirements make the VF iterations slower. In this chapter, an overview of Parallel Vector Fitting [16] to address this problem is given.

\subsection{Overview of formulation of multiport VF suitable for Parallel computing}

A brief overview of vector fitting scheme is presented in this section with formulation in the context of multiport structures. It also presents the parallelization strategy in the presence of multicore CPUs.

Consider a set of simulated or measured frequency samples $\boldsymbol{S}_{i j, k}=\boldsymbol{S}_{i j}\left(j \omega_{k}\right)$, with $k=1,2, \ldots, K$, where $K$ denotes the number of frequency samples, and $i, j=$ $1,2, \ldots, P$, where $P$ denotes the number of ports, as input to the VF algorithm. Corresponding multiport macromodel can be represented in the rational form as a 
sum of partial functions,

$$
\tilde{S}=\left[\begin{array}{cccc}
\tilde{S}_{11}(s) & \tilde{S}_{12}(s) & \ldots & \tilde{S}_{1 P}(s) \\
\tilde{S}_{21}(s) & \tilde{S}_{22}(s) & \ldots & \tilde{S}_{2 P}(s) \\
\vdots & \ldots & \ddots & \vdots \\
\tilde{S}_{P 1}(s) & \tilde{S}_{P 2}(s) & \ldots & \tilde{S}_{P P}(s)
\end{array}\right]
$$

with each element of the multiport macromodel represented as

$$
\tilde{S}_{i j}(s)=\sum_{n=1}^{N} \frac{\hat{c}_{i j, n}}{s-\overline{p_{n}}}+d+s e
$$

Here, $N$ denotes the number of poles. Vector Fitting method is used to find the set of unknown poles $\overline{p_{n}}$ and residues $\tilde{c}_{i j, n}$ that minimizes the least square distances $\sum_{k=1}^{K}\left|S_{i j, k}-\tilde{S}_{i j}\left(j \omega_{k}\right)\right|^{2}$. The nonlinear optimization problem (3.2) is solved by an iterative procedure that refines the initial set of poles $\overline{p_{n}}$. This is achieved by introducing scaling function with known poles $\overline{p_{n}}$ and unknown residues $c_{n}$.

$$
\sigma(s)=\sum_{n=1}^{N} \frac{c_{n}}{s-\overline{p_{n}}}+1
$$

The following condition is enforced by collecting all frequency responses $i, j=1$, $2, \ldots, P$ and all frequency samples $k=1,2, \ldots, K$.

$$
\sigma(s) S_{i j, k}=\sum_{n=1}^{N} \frac{\tilde{c}_{i j, n}}{s-\overline{p_{n}}}+d+s e
$$

The solution of (3.4) gives the residues $c_{n}$ of the scaling function and the residues $\tilde{c}_{i j, n}$. During the VF iterations, starting poles of $\sigma(s)$ are replaced with its zeros 
(i.e., $\overline{p_{n}} \leftarrow z_{n}$ ) and the process is repeated until convergence. The zeros of $\sigma(s)$ can be found out by computing the eigenvalues of the following system :

$$
z_{n} \leftarrow \operatorname{eig}\left(\boldsymbol{A}_{\sigma}-\boldsymbol{B}_{\sigma} \boldsymbol{C}_{\sigma} \boldsymbol{D}_{\sigma}^{-1}\right)
$$

where $\boldsymbol{A}_{\sigma}, \boldsymbol{B}_{\sigma}, \boldsymbol{C}_{\sigma}, \boldsymbol{D}_{\sigma}$ is a minimal state-space realization of $\sigma(s)$

$$
\begin{aligned}
& \boldsymbol{A}_{\sigma}=\left[\begin{array}{lll}
\bar{p}_{1} & & \\
& \ddots & \\
& & \bar{p}_{N}
\end{array}\right]_{N \times N} \\
& \boldsymbol{B}_{\sigma}=\left[\begin{array}{c}
1 \\
1 \\
\vdots \\
1
\end{array}\right]_{N \times 1} \\
& \boldsymbol{C}_{\sigma}=\left[\begin{array}{llll}
c_{1} & c_{2} & \ldots & c_{N}
\end{array}\right]_{1 \times N} \\
& \boldsymbol{D}_{\sigma}=[1]_{1 \times 1}
\end{aligned}
$$

$\overline{p_{n}}$ usually converges in few iterations. Once the poles have been found, (3.2) is used to determine the final residues for the multiport macromodel $\tilde{c}_{i j, n}$. 
For the case of multiport structure, closer look at (3.4) reveals a matrix structure

$$
\left[\begin{array}{ccccc}
\boldsymbol{\Phi}_{1} & 0 & \ldots & 0 & -S_{1} \boldsymbol{\Phi} \\
0 & \boldsymbol{\Phi}_{1} & \ldots & 0 & -S_{2} \boldsymbol{\Phi} \\
\vdots & \vdots & \ddots & \vdots & \vdots \\
0 & 0 & \ldots & \boldsymbol{\Phi}_{1} & -S_{P^{2}} \boldsymbol{\Phi}
\end{array}\right]\left[\begin{array}{c}
\tilde{c}_{1} \\
\vdots \\
\tilde{c}_{P^{2}} \\
\boldsymbol{c}
\end{array}\right]=\left[\begin{array}{c}
\boldsymbol{S}_{1} \mathbf{1}_{K} \\
\boldsymbol{S}_{2} \mathbf{1}_{K} \\
\vdots \\
\boldsymbol{S}_{P^{2}} \mathbf{1}_{K}
\end{array}\right]
$$

where

$$
\begin{aligned}
& \boldsymbol{\Phi}=\left[\begin{array}{ccc}
\frac{1}{s_{1}-\overline{p_{1}}} & \cdots & \frac{1}{s_{1}-\overline{p_{N}}} \\
\frac{1}{s_{2}-\overline{p_{1}}} & \cdots & \frac{1}{s_{2}-\overline{p_{N}}} \\
\vdots & \vdots & \vdots \\
\frac{1}{s_{K}-\overline{p_{1}}} & \cdots & \frac{1}{s_{K}-\overline{p_{N}}}
\end{array}\right]_{K \times N} \\
& \boldsymbol{\Phi}_{1}=\left[\begin{array}{lll}
\boldsymbol{\Phi} & \mathbf{1}_{K} & \boldsymbol{S}_{K}
\end{array}\right]_{K \times(N+2)} \\
& \mathbf{1}_{K}=\left[\begin{array}{llll}
1 & 1 & \ldots & 1
\end{array}\right]_{1 \times K}^{T} \\
& \boldsymbol{S}_{i+(P-1) j}=\operatorname{diag}\left(\boldsymbol{S}_{i j, k}\right) ; k \in 1,2, \ldots, K \\
& \tilde{\boldsymbol{c}}_{i+(P-1) j}=\left[\begin{array}{llll}
\tilde{c}_{i j, 0} & \tilde{c}_{i j, 1} & \ldots & \tilde{c}_{i j, N}
\end{array}\right]^{T}
\end{aligned}
$$

where $K$ is the total number of frequency samples, $P$ is the total number of ports and

$$
\boldsymbol{c}=\left[\begin{array}{llll}
c_{1} & c_{2} & \ldots & c_{N}
\end{array}\right]^{T}
$$

Here $c$ represents the vector containing the unknown residues of the scaling function. In [16], a computationally efficient approach to find $c$ was proposed. First, the matrix in each block row in (3.10) for $l=1,2, \ldots, P^{2}$ is considered indepen- 
dently and subjected to a QR decomposition as follows

$$
\boldsymbol{\Phi}_{1}-\boldsymbol{S}_{l} \boldsymbol{\Phi}_{1}=\boldsymbol{Q}_{l} \boldsymbol{R}_{l}=\left[\begin{array}{ll}
\boldsymbol{Q}_{l}^{a} & \boldsymbol{Q}_{l}^{b}
\end{array}\right]\left[\begin{array}{cc}
\boldsymbol{R}_{l}^{11} & \boldsymbol{R}_{l}^{12} \\
0 & \boldsymbol{R}_{l}^{22}
\end{array}\right]
$$

where the $(N \times N)$ upper triangular matrix $\boldsymbol{R}_{l}^{22}$ and $(2 \mathrm{~K} \times N)$ matrix $\boldsymbol{Q}_{l}^{b}$ are associated with the vector containing the unknown residues $(c)$ of the scaling function $\boldsymbol{\sigma}(s)$. By collecting all the $\boldsymbol{R}_{l}^{22}$ and $\boldsymbol{Q}_{l}^{b}$ for each of the block rows, we obtain a new compressed least square problem for evaluating $c$ as

$$
\left[\begin{array}{c}
\boldsymbol{R}_{1}^{22} \\
\boldsymbol{R}_{2}^{22} \\
\boldsymbol{R}_{3}^{22} \\
\vdots \\
\boldsymbol{R}_{P^{2}}^{22}
\end{array}\right]_{N P^{2} \times N}[\boldsymbol{c}]_{N \times 1}=\left[\begin{array}{c}
\boldsymbol{Q}_{1}^{b T} \boldsymbol{S}_{1} \mathbf{1}_{K} \\
\boldsymbol{Q}_{2}^{b T} \boldsymbol{S}_{2} \mathbf{1}_{K} \\
\vdots \\
\boldsymbol{Q}_{P^{2}}^{b T} \boldsymbol{S}_{P^{2}} \mathbf{1}_{K}
\end{array}\right]_{N P^{2} \times 1}
$$

The residues $c$ of the scaling function $\sigma(s)$ which are required in finding the zeros $z_{n}$ of the scaling function (which form the new set of poles for VF iterations) can be obtained efficiently using (3.18) and an additional QR.

\section{Relaxed VF}

In this section, a slightly modified version of VF which has superior convergence performance, especially in the presence of noise in the raw data [21]. The main idea is to remove the asymptotic unitary constraint of $\sigma(s)$ in (3.3) [22] by adding an asymptotic constant $d$ as an unknown. For this purpose, define a modified 
scaling function $\widehat{\boldsymbol{\sigma}}(s)$ as

$$
\widehat{\sigma}(s)=\sum_{n=1}^{N} \frac{c_{n}}{s-\overline{p_{n}}}+d
$$

In Relaxed VF [23], the least square system in (3.10) becomes

$$
\left[\begin{array}{ccccc}
\boldsymbol{\Phi}_{1} & 0 & \ldots & 0 & -S_{1} \boldsymbol{\Phi} \\
0 & \boldsymbol{\Phi}_{1} & \ldots & 0 & -S_{2} \boldsymbol{\Phi} \\
\vdots & \vdots & \ddots & \vdots & \vdots \\
0 & 0 & \ldots & \boldsymbol{\Phi}_{1} & -S_{P^{2}} \boldsymbol{\Phi}
\end{array}\right]\left[\begin{array}{c}
\tilde{c}_{1} \\
\vdots \\
\tilde{c}_{P^{2}} \\
\hat{\boldsymbol{c}}
\end{array}\right]=\left[\begin{array}{c}
0 \\
0 \\
\vdots \\
0 \\
0
\end{array}\right]
$$

where $\hat{c}$ represents the modified $\boldsymbol{c}$ in (3.16), by adding an asymptotic constant $d$ as,

$$
\hat{\boldsymbol{c}}=\left[\begin{array}{lllll}
d & c_{1} & c_{2} & \ldots & c_{N}
\end{array}\right]^{T}
$$

In order to avoid the all-vanishing trivial solution, one more equation is added

$$
R e \sum_{k=1}^{K}\left(d+\sum_{n=1}^{N} \frac{c_{n}}{s-\overline{p_{n}}}\right)=\boldsymbol{\beta}
$$

Correspondingly, the QR decomposition is applied similar to (3.17) leading to the 
modified set of equations for unknown residue computation as

$$
\left[\begin{array}{c}
\boldsymbol{R}_{1}^{22} \\
\boldsymbol{R}_{2}^{22} \\
\boldsymbol{R}_{3}^{22} \\
\vdots \\
\boldsymbol{R}_{P^{2}}^{22}
\end{array}\right] \hat{\boldsymbol{c}}=\left[\begin{array}{c}
0 \\
0 \\
\vdots \\
\boldsymbol{\beta}
\end{array}\right]
$$

Residues $\hat{\boldsymbol{c}}$ can be obtained using (3.23) and an additional QR.

\subsection{Splitting Strategies}

As it is evident from the formulation in the above section, the system of equations that need to be solved in every VF iteration becomes large with the increasing number of ports and poles. This leads to large size system whose solution requires significant CPU and memory resources. To address this, three splitting strategies [16] are discussed along with pole refinement function. If common pole assumption is used during vector fitting, it may become overly-restrictive leading to non-optimal accuracy. Another disadvantage is that, common pole assumption requires considering all responses together during the pole identification stage.

\subsubsection{None Splitting Strategy}

In this splitting strategy, the formulation of $\boldsymbol{A}_{i j}$ matrix (3.24) for each $\boldsymbol{S}_{i j}$ is done independently in separate CPUs. This is followed by QR factorization of the corresponding $\boldsymbol{A}_{i j}$ matrix in that particular CPU. This is then followed by the

residue evaluation (c) for the scaling function $\boldsymbol{\sigma}(s)$ for the entire $\boldsymbol{S}$ matrix on a 
single CPU (i.e., None Splitting). At the end of the final iteration, a common set of poles for the entire macromodel is obtained.

In Section 3.1, it is shown that the major part of the VF scheme in terms of floating point operations corresponds to the $\mathrm{QR}$ factorization of the following matrix $\boldsymbol{A}_{i j}$ (3.17),

$$
\boldsymbol{A}_{i j}=\boldsymbol{A}_{l}=\left[\boldsymbol{\Phi}_{1}-\boldsymbol{S}_{l} \boldsymbol{\Phi}_{1}\right]_{K \times(2 N+2)}
$$

where variable $l$ spans the multiport space; $l=1,2,3, \ldots, P^{2}$; with $l$ being expressed in terms of $i, j$ and $P$ as

$$
l=(i-1) P+j
$$

Prior to $\mathrm{QR}$ factorization, the $\boldsymbol{A}_{l}$ matrix (3.26) is split into real and imaginary parts and its QR factorization is obtained as

$$
\begin{gathered}
\boldsymbol{A}_{l}=\left[\begin{array}{c}
\operatorname{real}\left(\boldsymbol{A}_{l}\right) \\
\operatorname{imag}\left(\boldsymbol{A}_{l}\right)
\end{array}\right]_{2 K \times(2 N+2)}=\left[\begin{array}{ll}
\boldsymbol{Q}_{l} & \boldsymbol{R}_{l}
\end{array}\right]= \\
{\left[\begin{array}{ll}
\boldsymbol{Q}_{l_{2 K \times(N+2)}}^{a} & \boldsymbol{Q}_{l_{2 K \times N}}^{b}
\end{array}\right]_{2 K \times(2 N+2)}\left[\begin{array}{cc}
\boldsymbol{R}_{l_{(N+2) \times(N+2)}^{11}} & \boldsymbol{R}_{l_{(N+2) \times N}}^{12} \\
0 & \boldsymbol{R}_{l_{N \times N}}^{22}
\end{array}\right]_{(2 N+2) \times(2 N+2)}}
\end{gathered}
$$

Note that in (3.26), only $\boldsymbol{R}_{l}^{22}$ and $\boldsymbol{Q}_{l}^{b}$ are required for residue computation in (3.18). 
Detailed parallel execution steps for the None Splitting Strategy are outlined below:

(a) Each $\boldsymbol{A}_{l}$ matrix (i.e., $\boldsymbol{A}_{1}, \boldsymbol{A}_{2}, \ldots, \boldsymbol{A}_{l}, \ldots, \boldsymbol{A}_{P^{2}}$ ) of (3.26) is formed independently in separate CPUs. Let ' $T$ ' be the total number of available CPUs. For parallel formulation of each $\boldsymbol{A}_{l}$, they are assigned as follows

$$
\begin{cases}C P U_{1} \\ C P U_{2} \\ \vdots \\ C P U_{l} \\ \vdots \\ C P U_{T}\end{cases}
$$

Based on the available number of CPUs, assignment in (3.27) is executed for parallel computation in approximately $\frac{P^{2}}{T}$ loops.

(b) Once the matrix $\boldsymbol{A}_{l}$ is available, its $\mathrm{QR}$ factorization is done in the same CPU, to obtain $\boldsymbol{Q}_{l}$ and $\boldsymbol{R}_{l}$. Note that in (3.27), all $\boldsymbol{A}_{l}$ matrices have the same size and consequently there are no load balancing issues.

(c) Note that only the $(N \times N)$ upper triangular matrix $\boldsymbol{R}_{l}^{22}$ and $(2 K \times N)$ matrix $\boldsymbol{Q}_{l}^{b}$ are needed to evaluate the unknowns $\boldsymbol{c}$. By collecting only the $\boldsymbol{R}_{l}^{22}$ and $\boldsymbol{Q}_{l}^{b}$ for each of the $\boldsymbol{A}_{l}$ matrices, a new compressed least square problem is formed in one of the available CPUs, which will wait for all the CPU to compute their $\boldsymbol{R}_{l}^{22}$ and $\boldsymbol{Q}_{l}^{b}$. 
(d) As a last step in a given iteration, the residues (c) for the scaling function $\boldsymbol{\sigma}(s)$ are computed in one of the CPUs including the contributions from all the $\boldsymbol{A}_{l}$ matrices, using (3.18).

For the purpose of illustrating the above approach, consider a 4-port system with scattering matrix, $\boldsymbol{S}$, with individual elements that are represented using the definition in (3.24) as

$$
\left[\begin{array}{c}
\boldsymbol{S}_{11} \\
\vdots \\
\boldsymbol{S}_{14} \\
\boldsymbol{S}_{21} \\
\vdots \\
\boldsymbol{S}_{44}
\end{array}\right] \rightarrow\left[\begin{array}{c}
\boldsymbol{S}_{1} \\
\vdots \\
\boldsymbol{S}_{4} \\
\boldsymbol{S}_{5} \\
\vdots \\
\boldsymbol{S}_{16}
\end{array}\right]
$$

Let the available number of CPUs be 4. The solution process for the None Splitting approach for this example is illustrated in Figure 3.1. As seen, formulation and QR of individual matrices $\boldsymbol{A}_{1}, \boldsymbol{A}_{2}, \ldots, \boldsymbol{A}_{l} \ldots, \boldsymbol{A}_{16}$ are done parallely in a loop using the available CPUs. At the end, the residue computation for the scaling function for the entire $S$ matrix is done in a single CPU.

Major bottleneck in this approach is that the residue computation (c) needs to wait until all the $\boldsymbol{R}_{l}^{22}$ and $\boldsymbol{Q}_{l}^{b}$ are computed, which can result in a delay during the parallel pipeline scheduling. Also while computing QR for individual elements in the last loop of VF iteration, depending on the total number of CPUs and the total number of matrix elements $\boldsymbol{A}_{l}$ (i.e., $l \in 1,2,3, \ldots, P^{2}$ ), some of the CPUs may remain idle based on the corresponding $\frac{P^{2}}{T}$ value. 


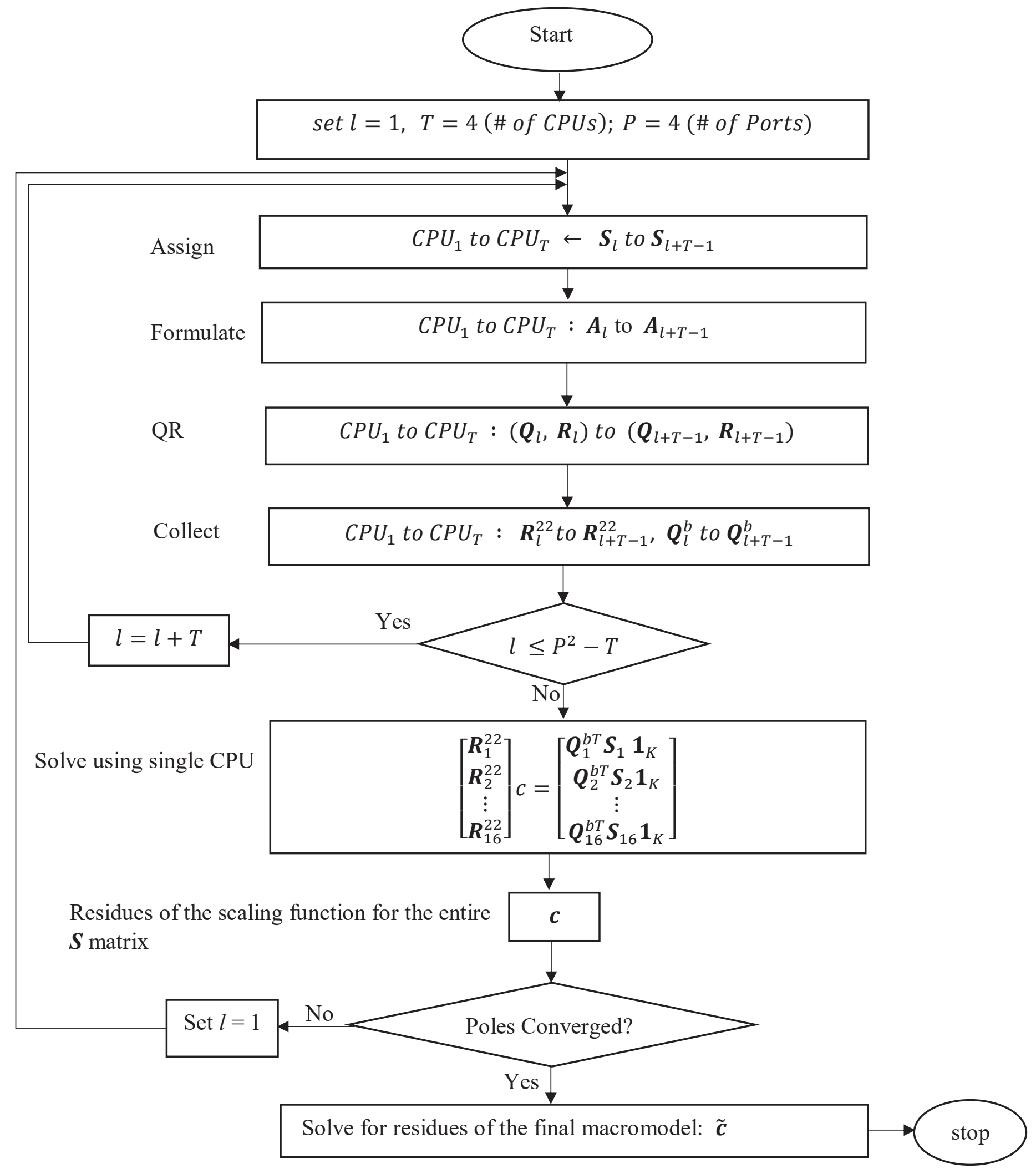

Figure 3.1: Graphical Illustration of None Splitting Strategy for multiport VF 


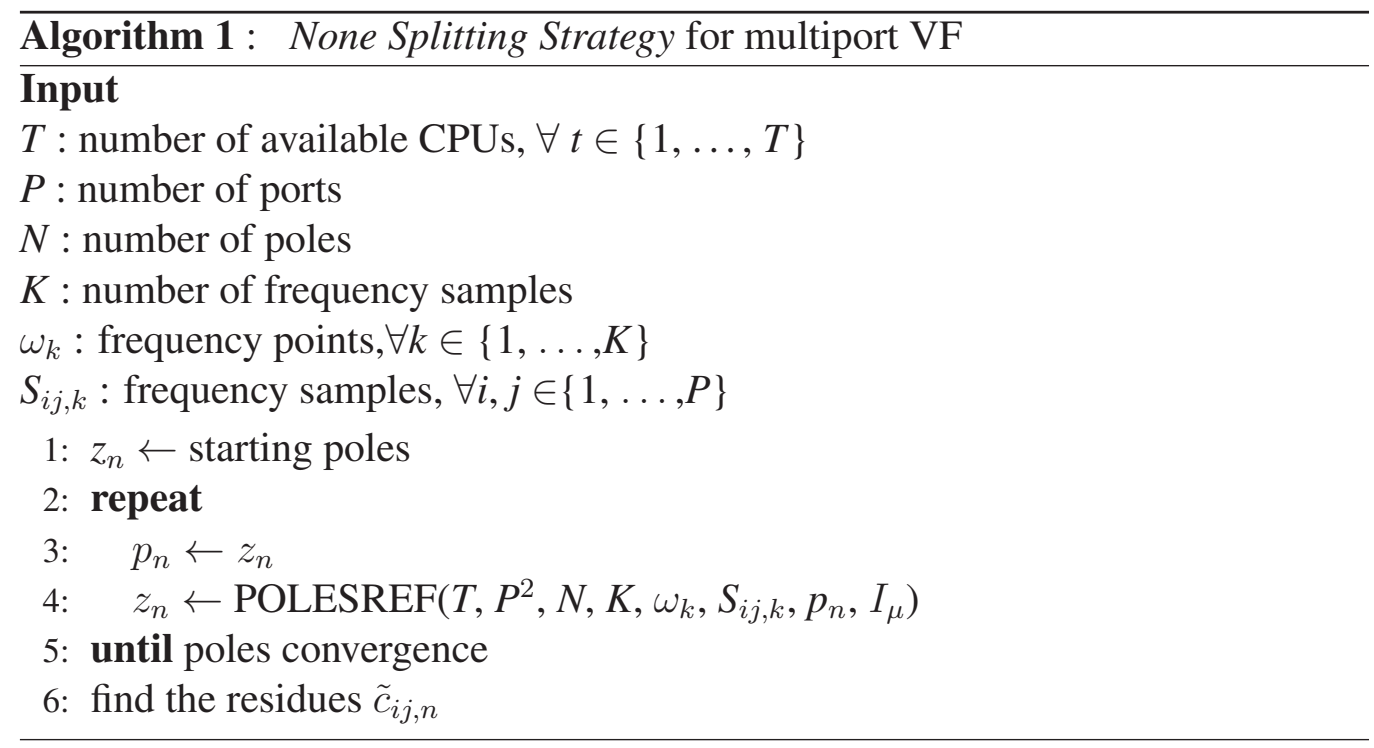

The pseudocode corresponding to the None Splitting Strategy is given in Algorithm 1. The function POLESREF inside Algorithm 1 accepts the initial set of poles $p_{n}$ and raw frequency sample data points. It outputs $z_{n}$ (zeros of the scaling function which are required in calculating the relocated poles) using $T$ number of CPUs in parallel. The pseudocode for the POLESREF function is given in Algorithm 2. 


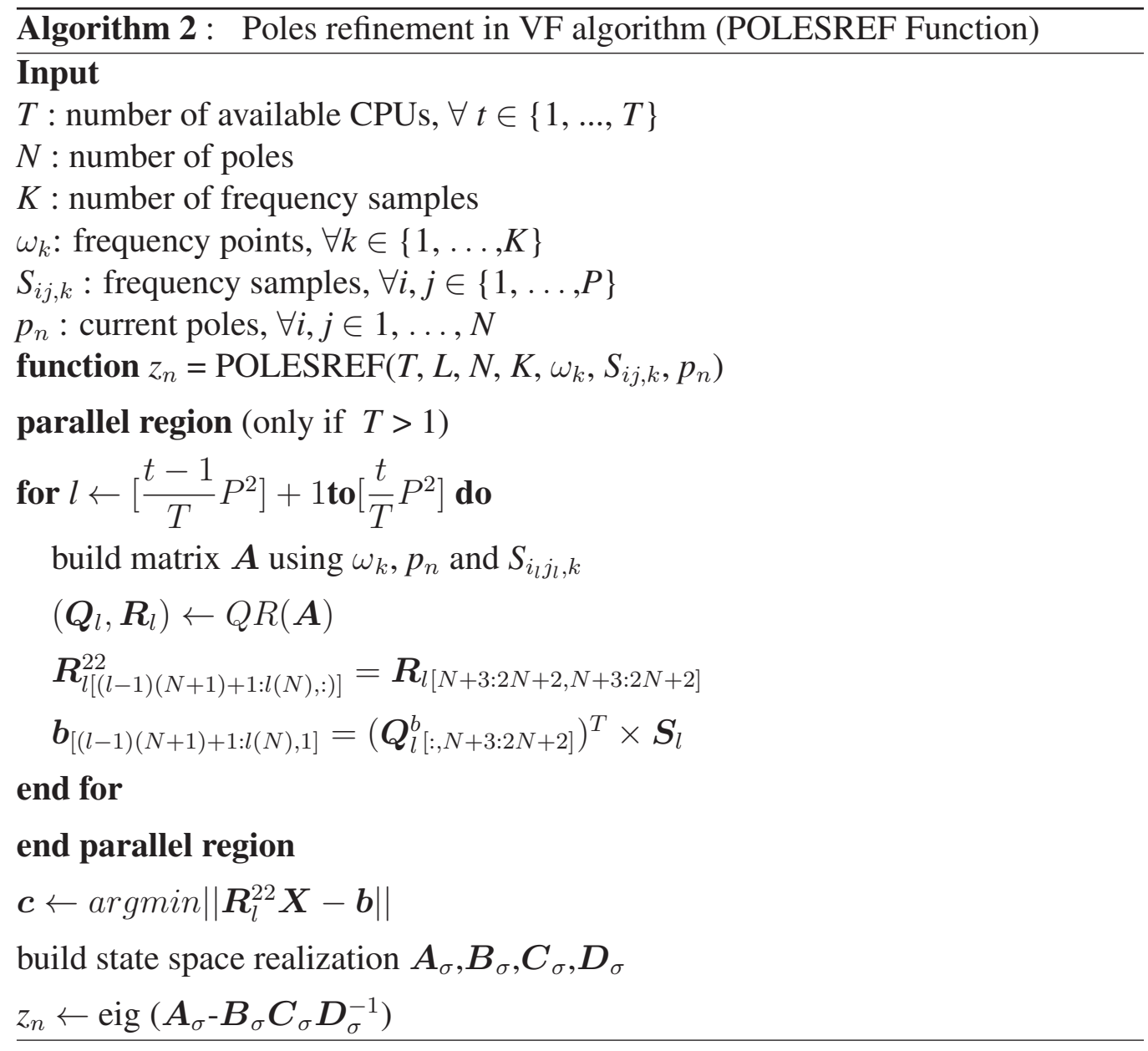

\subsubsection{All Splitting Strategy}

In this approach, scaling function for each of the $\boldsymbol{S}$ matrix element $\left(\boldsymbol{S}_{i j}\right)$ is considered independently. Also each $\boldsymbol{S}_{i j}$ element is considered to have its own set of poles. In this splitting scheme, the formulation, QR factorization and the residue evaluation of $\boldsymbol{\sigma}_{i j}(s)$ for each $\boldsymbol{A}_{l}$ matrix (3.26) is done independently in separate CPUs. Here, matrix $\boldsymbol{A}_{l}$ (i.e., $\boldsymbol{A}_{1}, \ldots, \boldsymbol{A}_{l}, \ldots, \boldsymbol{A}_{P^{2}}$ ) (3.26) is formulated on separate CPUs. Also in each of the respective CPUs, QR factorization as well as the 
residues of the scaling function $\boldsymbol{\sigma}_{l}(s)$ (which is specific to the corresponding $\boldsymbol{A}_{l}$ in this approach) are evaluated. At the end, individual pole sets as well as their residues are computed for each of the $\boldsymbol{S}_{i j}$ element.

The performance of this strategy tends to be better than the None Splitting approach and the performance tends to increase with the increase in the number of ports $P$. This is because any delay waiting for all $\boldsymbol{R}_{l}^{22}$ are eliminated in this approach.

The Parallel execution steps for the All Splitting Strategy are outlined below.

(a) Each $\boldsymbol{A}_{l}$ matrix (i.e., $\boldsymbol{A}_{1}, \boldsymbol{A}_{2}, \ldots, \boldsymbol{A}_{l}, \ldots, \boldsymbol{A}_{P^{2}}$ ) of (3.26) is formed independently in separate CPUs. For parallel formulation of each $\boldsymbol{A}_{l}$ matrix, it is assigned in the same way as in (3.27).

(b) Once the matrix $\boldsymbol{A}_{l}$ is available, it is followed by immediate QR factorization in the same CPU. Next $\boldsymbol{R}_{l}^{22}$ and $\boldsymbol{Q}_{l}^{b}$ are collected and then the residues of the corresponding scaling function $\boldsymbol{\sigma}_{l}(s)$ (which is specific to that $\boldsymbol{S}_{i j}$ element) is computed in the same CPU, using the relation

$$
\left[\boldsymbol{R}_{l}^{22}\right]_{N \times N}\left[\boldsymbol{c}_{l}\right]_{N \times 1}=\left[\boldsymbol{Q}_{l}^{b T} \boldsymbol{S}_{l} \mathbf{1}_{K}\right]_{N \times 1}
$$

(c) Similarly, the above step is used for the computation of the residues for all the $\boldsymbol{S}_{i j}$ elements independently.

(d) Based on the available number of CPUs, steps (a), (b) and (c) are executed for computing the residues of rest of the $\sigma_{l}(s)$ in approximately $\frac{P^{2}}{T}$ loops. 
For the purpose of illustrating the above approach, consider a 4-port system with scattering matrix, $S$, and with individual elements $S_{11}, S_{12}, S_{13}, S_{14}, \ldots, S_{44}$ or $S_{1}, S_{2}, \ldots, S_{l} \ldots, S_{16}$ as per the definitions in (3.25). Let the available number of CPUs be 4. The solution process for this example using the All Splitting approach for this example is illustrated in Figure 3.2. As seen, formulation, QR and residue evaluation of individual matrices $\boldsymbol{A}_{1}, \boldsymbol{A}_{2}, \ldots, \boldsymbol{A}_{l} \ldots, \boldsymbol{A}_{16}$ are done parallely in a loop using the available CPUs.

The minor bottleneck in this approach is the memory management, when the number of CPUs working in parallel is more, some CPUs may run out of memory. These CPUs call for memory management and have to wait until the memory is released. This can be overcome by imposing implicit barriers that may block the execution of the CPU until memory is released. This overhead is typically negligible considering the overall runtime. 


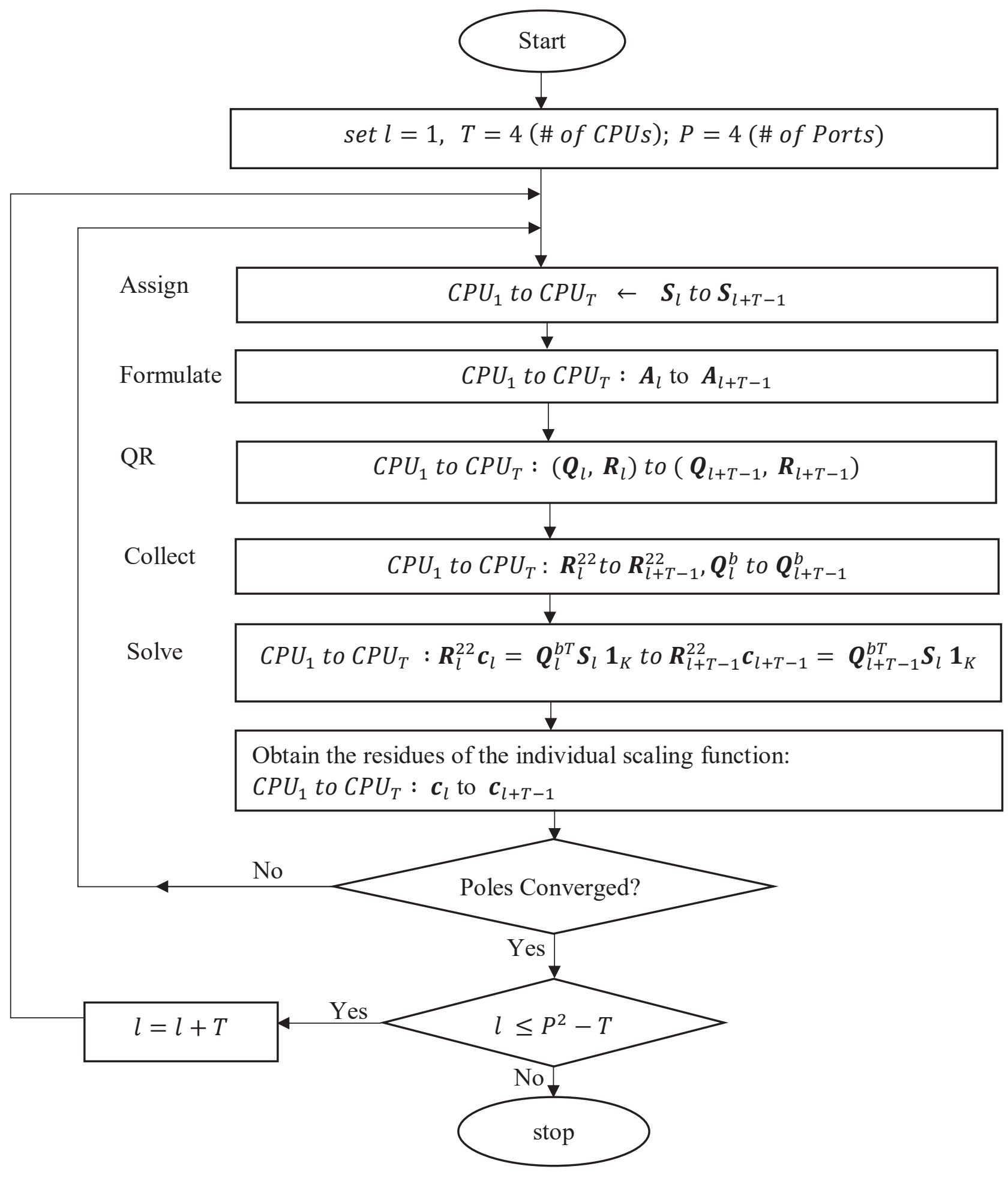

Figure 3.2: Graphical Illustration of All Splitting Strategy for multiport VF 
A pseudocode corresponding to the All Splitting Strategy is given in Algorithm 3.

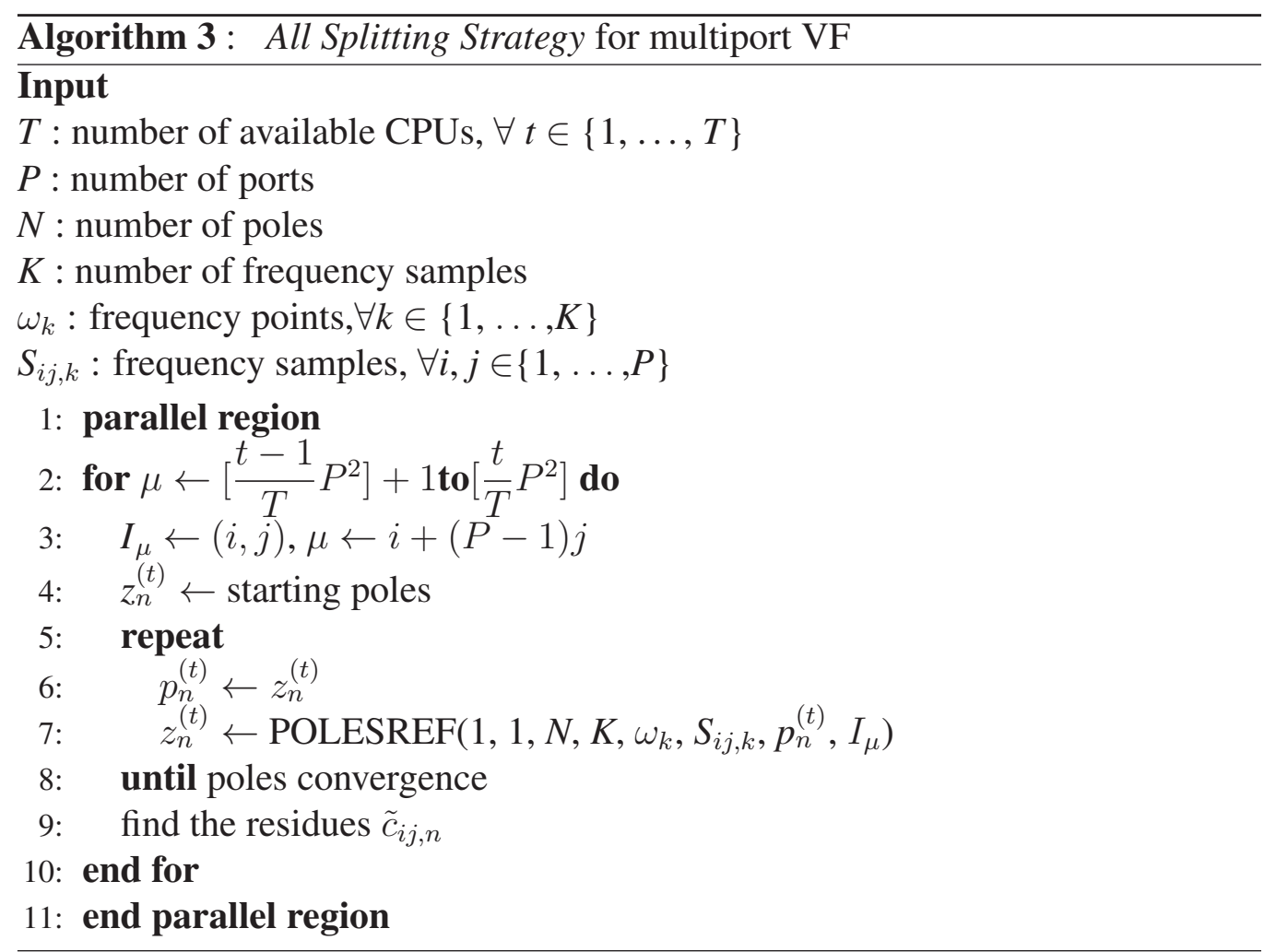

\subsubsection{Column Splitting}

This approach provides parallelization performance that ranges between that of All Splitting and None Splitting approaches. In this approach, a specific scaling function is considered for each column unlike the None Splitting Strategy which considers one scaling function for the entire matrix or the All Splitting Strategy which considers individual scaling functions for each element. In this splitting scheme, each column is assigned to a particular CPU. This CPU performs the formulation of each $\boldsymbol{A}_{l}$ matrix in that column, its $\mathrm{QR}$ decomposition and stores 
the corresponding $\boldsymbol{R}_{l}^{22}$ and $\boldsymbol{Q}_{l}^{b}$. Finally, it computes a common $\boldsymbol{c}_{j}$, which are the residues of the scaling function that is specific for that column.

For the purpose of illustrating the above approach, consider a 4-port system with scattering matrix. Let the available number of CPUs be 4. Following steps are considered during the parallel execution based on their columns.

(a) Assign each $\boldsymbol{S}_{i j}$ based on their columns to different CPUs for formulating the corresponding $\boldsymbol{A}_{l}$ matrices (3.26) as follows;

$$
C P U_{1}\left\{\begin{array}{l}
\boldsymbol{A}_{11} \\
\boldsymbol{A}_{21} \\
\boldsymbol{A}_{31} \\
\boldsymbol{A}_{41}
\end{array} ; C P U_{2}\left\{\begin{array}{l}
\boldsymbol{A}_{12} \\
\boldsymbol{A}_{22} \\
\boldsymbol{A}_{32} \\
\boldsymbol{A}_{42}
\end{array} ; C P U_{3}\left\{\begin{array}{l}
\boldsymbol{A}_{13} \\
\boldsymbol{A}_{23} \\
\boldsymbol{A}_{33} \\
\boldsymbol{A}_{43}
\end{array} ; C P U_{4}\left\{\begin{array}{l}
\boldsymbol{A}_{14} \\
\boldsymbol{A}_{24} \\
\boldsymbol{A}_{34} \\
\boldsymbol{A}_{44}
\end{array}\right.\right.\right.\right.
$$

(b) This is followed by an independent QR decomposition of these matrices, collecting the corresponding $\boldsymbol{R}_{l}^{22}$ and $\boldsymbol{Q}_{l}^{b}$ matrices and then computing residues of the scaling function that is specific to that column, in the same CPU that was used in step (a).

The solution process for the Column Splitting approach for this example is illustrated in Figure 3.3. As seen, formulation, QR and residue evaluation of the scaling function for each column of $S$ matrix is done parallely using the available CPUs.

It is to be noted that, this strategy can experience pipelining bottle neck. This 
is because, since a particular CPU is used for formulation, QR computation for all the $\boldsymbol{A}_{l}$ matrices in a column, as well as residue computation of the scaling function for that column, the CPU needs to wait until all the $\boldsymbol{R}_{l}^{22}$ and $\boldsymbol{Q}_{l}^{b}$ for all the $\boldsymbol{A}_{l}$ matrices of that column are computed. This adds to the delay during the parallel execution. 


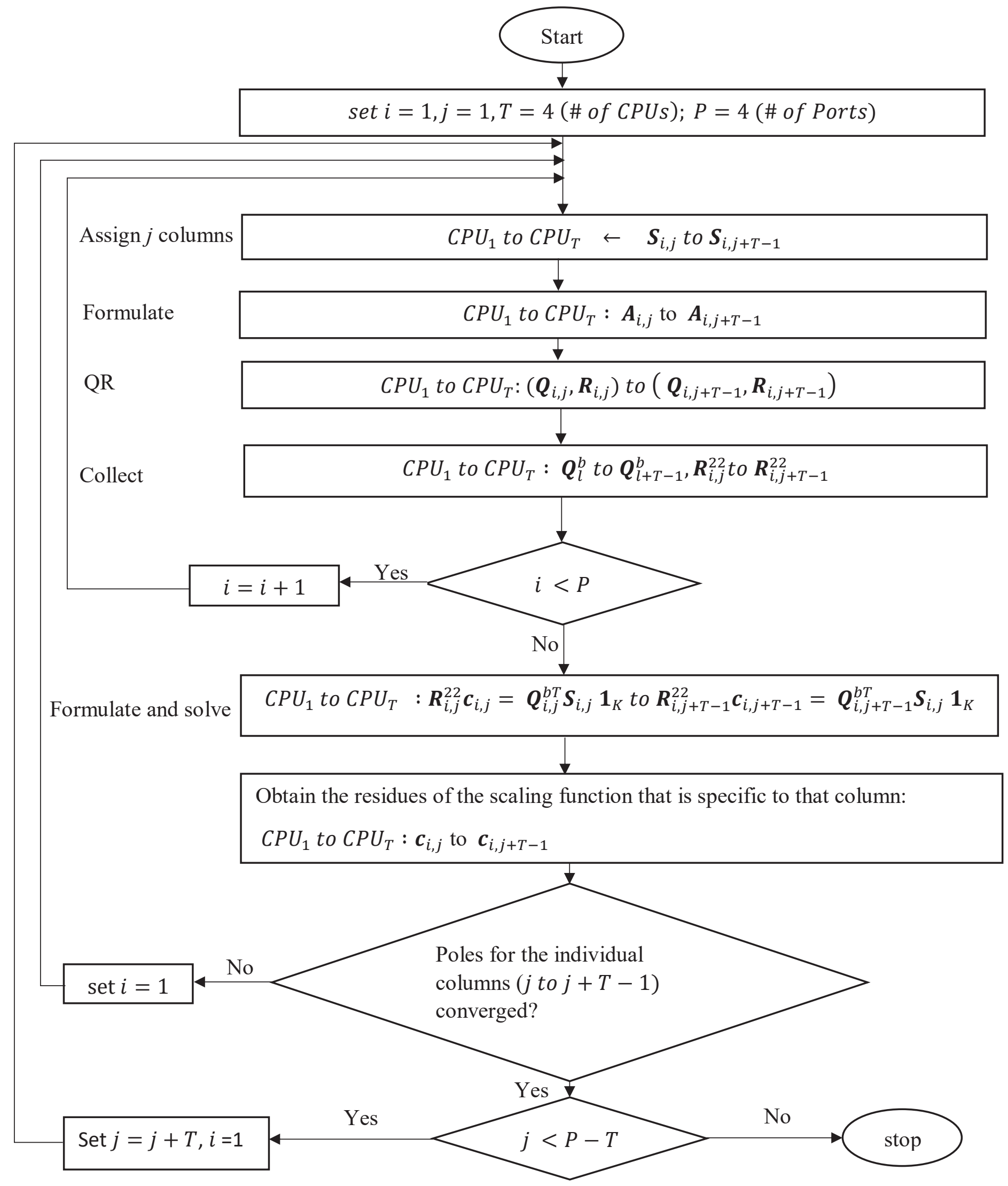

Figure 3.3: Graphical Illustration of Column Splitting Strategy for multiport VF 
The pseudocode corresponding to the Column Splitting Strategy is given in Algorithm 4 .

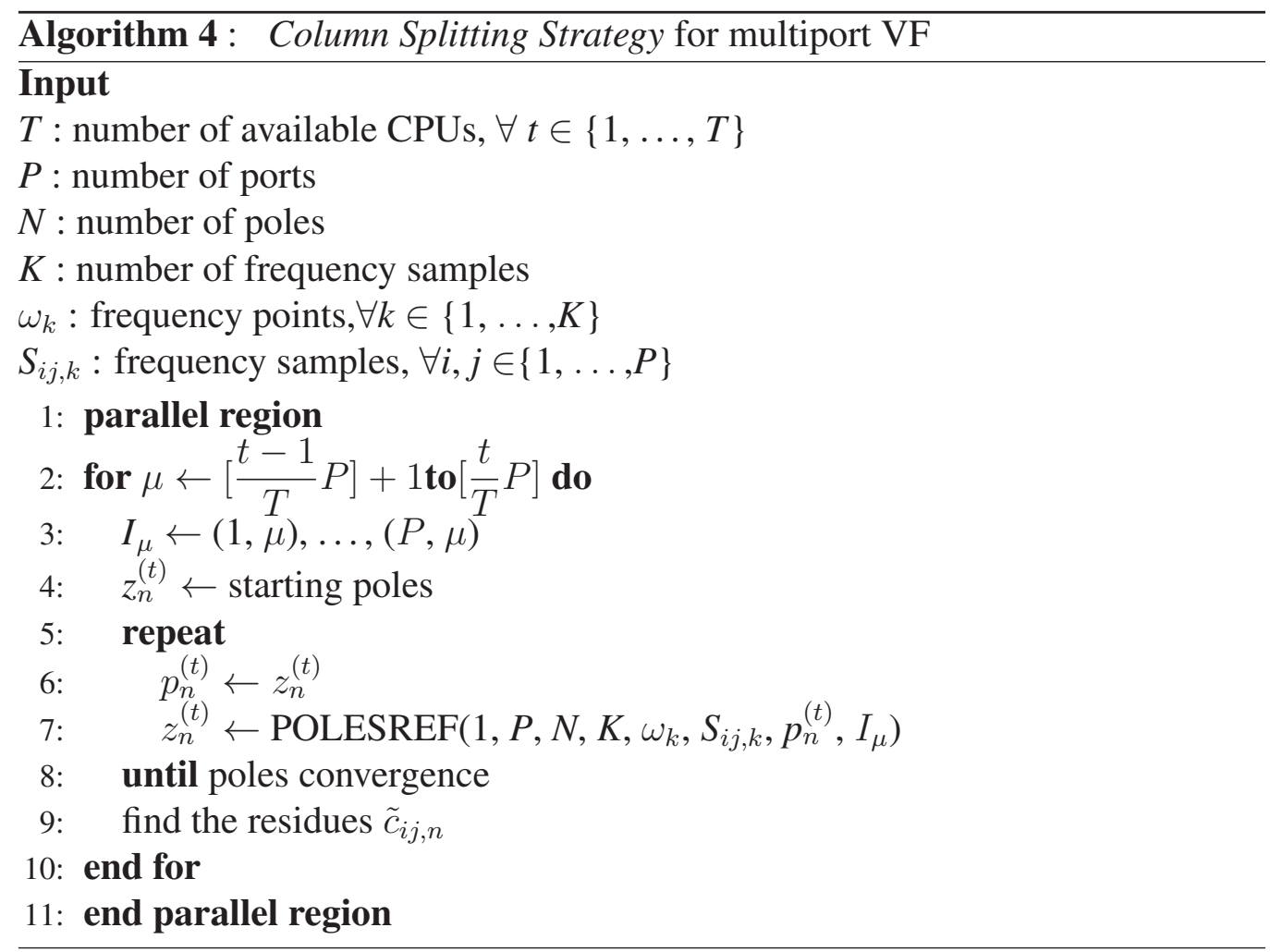




\section{Chapter 4}

\section{Review of GPU Architectures and Programming}

\subsection{Introduction}

Many numerical and scientific computing applications require the solution of a large linear algebraic system of equations, which can be computationally expensive. This has led to use of Graphics Processing Unit (henceforth referred to as GPU in this thesis) which is fast emerging as one of the preferred computing platforms. GPU is traditionally used to accelerate computation in graphics and video applications. The first GPU released in 1999, was used for the purpose of graphics processing. Since the release of CUDA in 2006, GPU quickly became a preferred computing platform in many scientific applications due to its massively parallel architecture (owing to thousands of cores) and large memory bandwidth. Since then, GPU has been advancing to handle many innovative applications emerging from different fields such as artificial intelligence [17], integrated circuit analysis [18] and medical imaging [19], etc.

\subsection{GPU Architecture}

This section describes the main characteristics of the GPU architecture, including programming model, memory hierarchy, and special features relevant to the 
proposed work. A generic architecture for the CUDA Programming model is described in Figure 4.1.

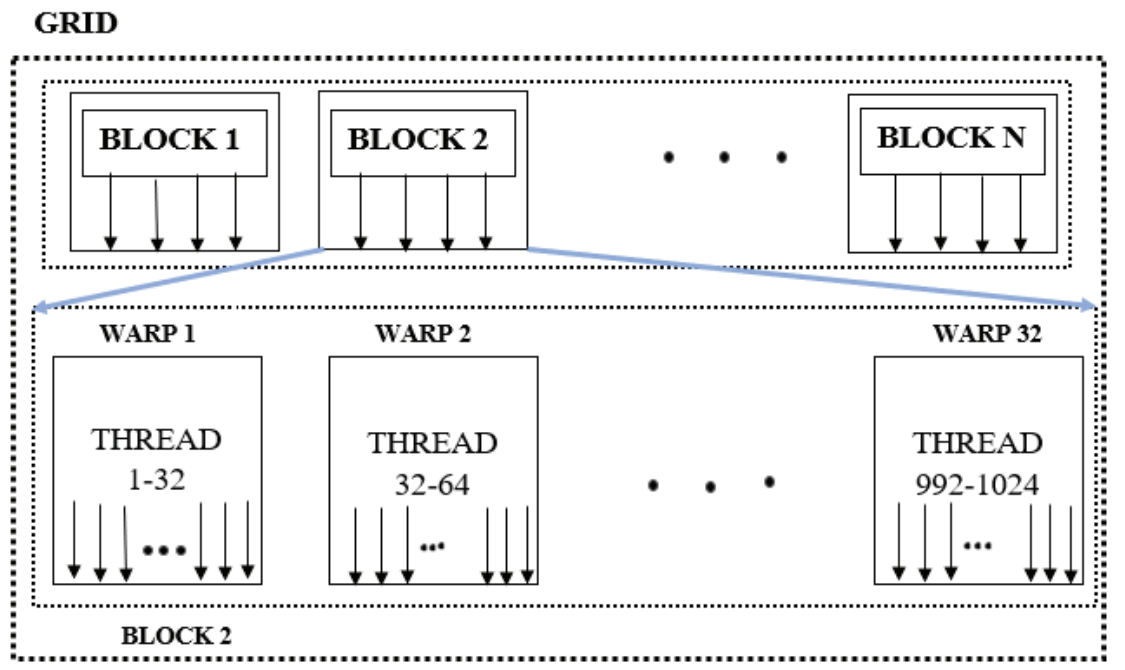

Figure 4.1: CUDA Programming Model: Grid, Block, Warp, Thread Architecture

\subsubsection{Terminology}

Thread, block, Grid, Warp, Kernel are the important terminologies in GPU. The GPU program is a parallel program to be run on kernel. Several primitive 'threads' will simultaneously execute a kernel program. Batches of these primitive threads are organized into 'thread blocks'. The thread block is chosen based on the desired amount of shared memory and memory latency.

In GPU architecture, a block consists of multiple threads (up to a maximum size of 1024 threads). The thread is the smallest grain of the hierarchy device. A grid consists of multiple blocks (up to a maximum of 1024 blocks). A grid is a 
collection of thread blocks of same thread dimensionality which all execute the same kernel. Threads within a block are further grouped into warps (32 threads form a warp), which is the scheduling unit in a GPU hardware. All threads within the same warp are scheduled to execute the same instruction. Threads and blocks are indexed individually using the built-in variables (threadIdx.x and blockIdx.x). Threads are conceptually organized into 1-D, 2-D, 3-D array of threads for user convenience.

\subsubsection{Memory Hierarchy}

GPU memory is divided into on-chip memory and off-chip memory which have huge difference in performance. The memory that reside on the GPU chip are register and shared memory. The local, global, constant, and texture memory reside off-chip. Each type of memory on the device has its own advantages and disadvantages. If available memory is used incorrectly in the application, it can degrade performance.

Comparing in terms of speed, registers are the fastest, followed by the shared memory, constant memory and texture memory. Local memory and global memory are the slowest. Therefore, the best performance can be achieved if more of registers, shared and constant memories are used.

This memory hierarchy in GPU is described in Figure 4.2. 


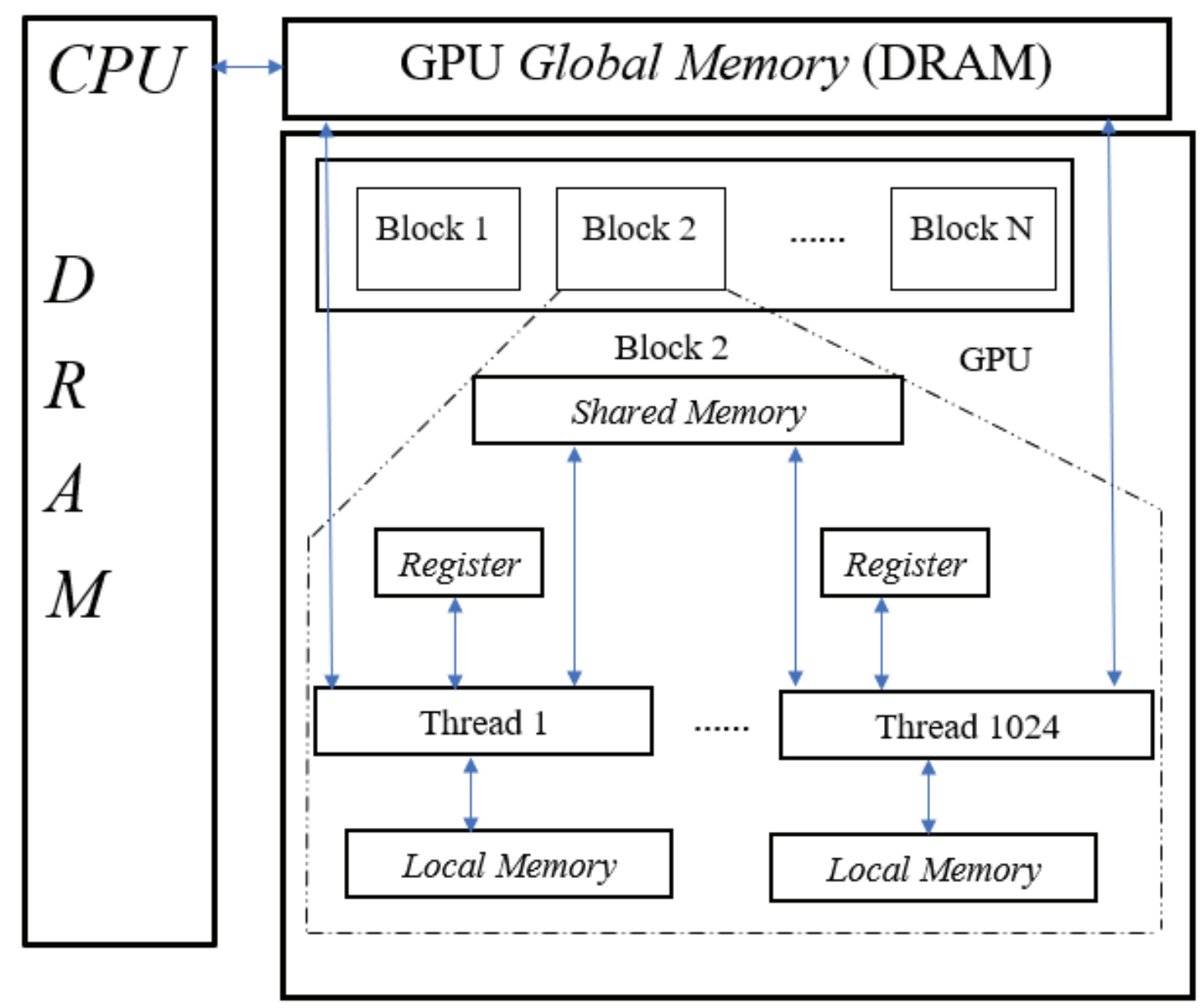

Figure 4.2: Cuda Memory Architecture

\section{Registers}

Registers are on-chip and are the fastest memory in GPU. However, they are very limited in size and only accessible locally by each thread. Therefore, data stored in register memory is visible only to that thread and lasts only for the lifetime of that thread. If a kernel uses more registers than its allowed limit, the compiler 
will allocate these extra register usages into local memory that resides in the slow global memory but cached at L1 cache for faster access.

\section{Shared memory}

Shared memory is an on-chip memory accessible by all threads within the same thread block. Data inside shared memory lasts for the duration of the block. Shared memory is usually used as a user-managed cache for High-Performance Computing (HPC). Shared memory usage needs to be designed carefully to avoid bank conflicts, to provide faster access speed. As discussed, when there are no bank conflicts, shared memory performance is comparable to register memory.

\section{Global memory}

Global memory is placed outside the chip and it provides the largest storage capacity. The data used for computation is usually transmitted from CPU DRAM to GPU Global memory (which is also a DRAM). Once the GPU computes the results, they are transferred back to CPU DRAM. Here, the transfer of data between the GPU and CPU happens only through their respective DRAMs. Global memory is visible to all the threads within the application (including the host) and has a lifetime of the allocating program (or until CudaFree is called). Shared memory needs to be accessed in a coalesced manner. Depending on how coalesced the memory access is, the speed up can be brought depending on the card and its memory interface. However, it has the slowest performance characteristics. 


\section{Local memory}

Local memory is not a physical type of memory, but an abstraction of global memory. Its scope is local to the thread and it resides off-chip, which makes it as expensive to access as global memory. Local memory is used only to hold automatic variables. The compiler makes use of local memory when it determines that there is not enough register space to hold the variable. Automatic variables that are large structures or arrays are also typically placed in local memory. Lifetime of local memory is also equal to the lifetime of the thread.

\section{Constant memory}

Constant memory is a cached global memory. Constant memory is beneficial for data that will not change over the course of a kernel execution and that is readonly. Using constant memory rather than global memory can reduce the required memory bandwidth. However, this performance gain can only be realized when a warp of threads reads the same location. They are accessible by all the threads and has a lifetime of the application.

\section{Texture Memory}

Texture memory is a cached global memory like constant memory. It is another variety of read-only memory on the device. When all reads in a warp are physically adjacent, using texture memory can reduce memory traffic and increase performance compared to global memory. 


\subsection{CUDA Programming Model}

In this model, the Runtime Application Program Interface (henceforth referred to as API in this thesis) is used for managing host/device interfacing, the thread hierarchy identification variables, and several miscellaneous identifiers. The highest level of control is provided by the function type identifiers. Three such identifiers are the host, global and device. The host identifier denotes a function to be run on the general purpose CPU or host. This is the default type, and therefore is not explicitly used. Host functions do everything a normal $\mathrm{C}++$ function does and call the Runtime API functions. These function identifiers are primarily concerned with memory management (which includes cudaMalloc, cudaFree and cudaMemcpy). Standard pointers need to be used in the host identifier. Here, the pointer value is an address on the device memory rather than the host system memory. In the following, a brief description of the syntax for cudaMalloc, cudaFree and cudaMemcpy is presented.

\subsection{1 cudaMalloc}

cudaMalloc allocates size bytes of linear memory on the device and returns *devPtr (a pointer to the allocated memory). The allocated memory is for any kind of variable and is not cleared. cudaMalloc returns cudaErrorMemoryAllocation in case of failure.

$$
\text { cudaError_tcudaMalloc(void } * * d e v P t r \text {, size_t size) }
$$




\subsection{2 cudaFree}

cudaFree frees the memory space pointed to by devPtr (devPtr is a pointer to memory to free) which must have been returned by a previous call to cudaMalloc. If cudaFree (devPtr), has already been called before, an error is returned, If devPtr is 0 , no operation is performed. In case of failure, cudaFree returns cudaErrorInvalidDevicePointer .

$$
\text { cudaError_t cudaFree(void*devPtr) }
$$

\subsubsection{Kernel Call}

Another Runtime API function of importance is kernel invocation. The syntax KernelFunctionName $<<<$ GridDim,ThreadBlockDim $>>>$ (@params) is used to specify the dimensions of the thread block and grid of thread blocks. The kernel function is then run on the device and any memory allocated using cudaMalloc can be communicated by passing the device pointer as a parameter. Kernel functions use the global identifier. This denotes which functions may be called from the host on the device.

\subsubsection{Data transfer between host memory and device memory}

CudaMemcpy copies the count (size in bytes to copy) bytes from the memory area pointed to by src (source memory address) to the memory area pointed to by dest (destination memory address). cudaMemcpy kind is either cudaMemcpy- 
HostToDevice or cudaMemcpyDeviceToHost which specifies direction of copy.

$$
\begin{array}{r}
\text { cudaError_t cudaMemcpy(void* dst, const void* src, } \\
\text { size_t count, enum cudaMemcpyKind kind) }
\end{array}
$$

The final identifier device are functions that run on the device and not be called from the host. The typical use of the host identifier is when a function is needed by both the host and the device. However, both the device and host identifiers are used to instruct the compiler to construct both versions.

Within global and device functions, several system variables are used to manage threads and provide unique identification. For example, threadIdx is a dim3 type with the unique identification of a thread within a thread block. Likewise, blockIdx provides a unique identification of a thread block within a grid. With these identifiers and the syncthreads(), it syncs all the threads in a thread block, execution can be effectively managed. 


\section{Chapter 5}

\section{GPU based Parallel VF}

In Chapter 3, a brief review of parallel vector fitting [16] and its splitting strategies for high-order rational-function approximation of multiport tabulated data was presented. When the number of input-output ports $(P)$ of the structure becomes large, excessive computational requirements make the VF iterations slower. As noted in Chapter 3, the major cost of the VF algorithm is associated with the QR factorization of the $\boldsymbol{A}_{i j}$ matrices (3.26). Parallel VF [16] attempted to address this by considering multicore or distributed CPU environment.

In this chapter, we will further advance the field by considering the emerging platform of GPUs for VF and the necessary parallelization strategies for the same. Several splitting strategies based on None Splitting Strategy and All Splitting Strategy that are suitable for GPU platforms along with the multicore CPUs are presented and the performance of the VF algorithm is investigated. Column Splitting Strategy is not considered as it often leads to CPU idle times based on the number of CPUs and the number of columns.

In developing the mixed CPU and GPU algorithm, emphasis is placed on minimizing the major cost associated with the VF algorithm, which is the QR factorization of the individual $\boldsymbol{A}_{l}$ elements. Also, since the data transfer time between the CPU 
and GPU takes significant amount of time, the developed approaches will explore various options to minimize the same. Hence, formulation of the $\boldsymbol{A}_{l}$ elements were planned on the CPUs while QR factorization and scaling function residue computations were considered on mixed CPU and GPU platforms in the proposed algorithms.

\subsection{Approach-1 : Formulation of $A_{i j}$ in CPU followed by immediate $Q R$ fac- torization and residue evaluation in GPU for None Splitting Strategy}

In this splitting strategy, the formulation of $\boldsymbol{A}_{l}$ matrix (3.26) for each $\boldsymbol{S}_{i j}$ is done independently in separate CPUs. This is followed by immediate QR factorization of the corresponding $\boldsymbol{A}_{l}$ matrix in a GPU which also stores the $\boldsymbol{R}_{l}^{22}$ and $\boldsymbol{Q}_{l}^{b}$ matrices. Next, the residue evaluation for the scaling function $\boldsymbol{\sigma}(s)$ for the entire $\boldsymbol{S}$ matrix is performed in GPU itself. At the end of the final iteration, a common set of poles for the entire macromodel is obtained.

\subsubsection{Approach-1 with multiple CPUs and a single GPU}

Parallel execution steps for the proposed Approach-1 with multiple CPUs and a single GPU are outlined below.

(a) Each $\boldsymbol{A}_{l}$ matrix (i.e., $\boldsymbol{A}_{1}, \boldsymbol{A}_{2}, \boldsymbol{A}_{3}, \ldots, \boldsymbol{A}_{P^{2}}$ ) of (3.26) is formed independently in separate CPUs. Let ' $T$ ' be the number of available CPUs. For parallel formulation of each $\boldsymbol{A}_{l}$, we assign them as in (3.27). Based on the available number of CPUs, assignment in (3.27) is executed for parallel computation in approximately $\frac{P^{2}}{T}$ loops. 
(b) Once the $\boldsymbol{A}_{l}$ matrix is available, it is sent to the GPU immediately for QR factorization to obtain $\boldsymbol{Q}_{l}$ and $\boldsymbol{R}_{l}$. (i.e., although each $\boldsymbol{A}_{l}$ matrix is formulated parallely, it is sent to GPU in a serial way while the QR factorization of a single $\boldsymbol{A}_{l}$ matrix of a particular CPU is being computed, the remaining CPUs needed to wait until the QR computation of that $\boldsymbol{A}_{l}$ matrix is completed in the GPU).

(c) Next, the $\boldsymbol{R}_{l}^{22}$ and $\boldsymbol{Q}_{l}^{b}$ matrices are collected for each of the $\boldsymbol{A}_{l}$ matrices in the same GPU one at a time and the residues $(c)$ for the scaling function $\boldsymbol{\sigma}(s)$ are computed in that GPU itself, including the contributions of all $\boldsymbol{A}_{l}$ matrices, using (3.18).

For the purpose of illustrating the above approach, consider a 4-port system with scattering matrix, $\boldsymbol{S}$, with individual elements that are represented as in (3.28). Let the available number of CPUs be 4 and GPU be 1 . The solution process for the Approach-1 (i.e., Approach-1 with multiple CPUs and a single GPU) for this example is illustrated in Figure 5.1. As seen, formulation of individual matrices $\boldsymbol{A}_{1}, \boldsymbol{A}_{2}, \ldots, \boldsymbol{A}_{l} \ldots, \boldsymbol{A}_{16}$ are done parallely in CPU, followed by immediate QR factorization in the GPU. At the end, the residue computation for the scaling function $\boldsymbol{\sigma}(s)$ for the entire $\boldsymbol{S}$ matrix is done in that GPU itself. The pseudocode corresponding to the Approach-1 is given in Algorithm 5. 


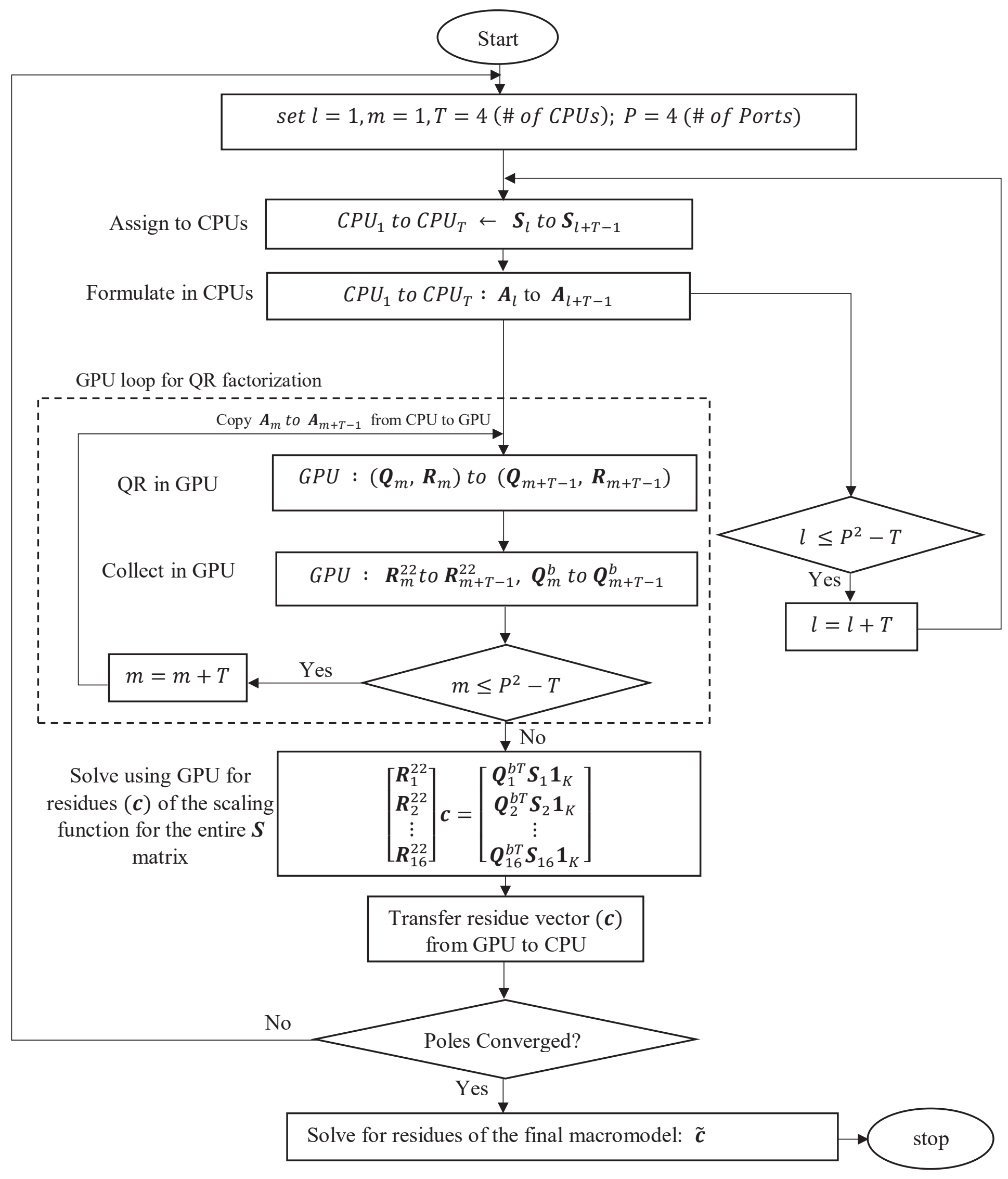

Figure 5.1: Graphical Illustration of Approach-1 for multiport VF 


\subsubsection{Discussion on CPU to GPU, GPU to CPU Data transfer time for Approach- 1}

In this subsection, a discussion comparing the transfer time for CPU to GPU, GPU to $\mathrm{CPU}$ and actual GPU to CPU, is given.

The data transfer time and computing time reported in this chapter are based on the execution using the Compute Canada platform (a national computing grid) [24]. This platform has the module configuration of four CPU cores (Intel Xeon E52650), 64-GB RAM, and a GPU. The GPU (Pascal P100) consist of 3584 cores, $4096 \mathrm{~KB}$ of L2 cache memory, and 12-GB RAM and operates at $1.328 \mathrm{GHz}$ clock frequency.

Note that, typical applications of VF include large number of frequency samples ( $\approx 1000$ ), port numbers varying between 2 to 150 (such as in IC packages) and larger bandwidth which often requires between few poles to 100 poles for accurate characterization. Hence the examples considered in the thesis were taken in this typical scenario of VF application in electronic designs. Correspondingly, considered $\boldsymbol{A}_{l}$ matrix sizes are in the range of $(100 \times 100)$ to $(4000 \times 100)$. 


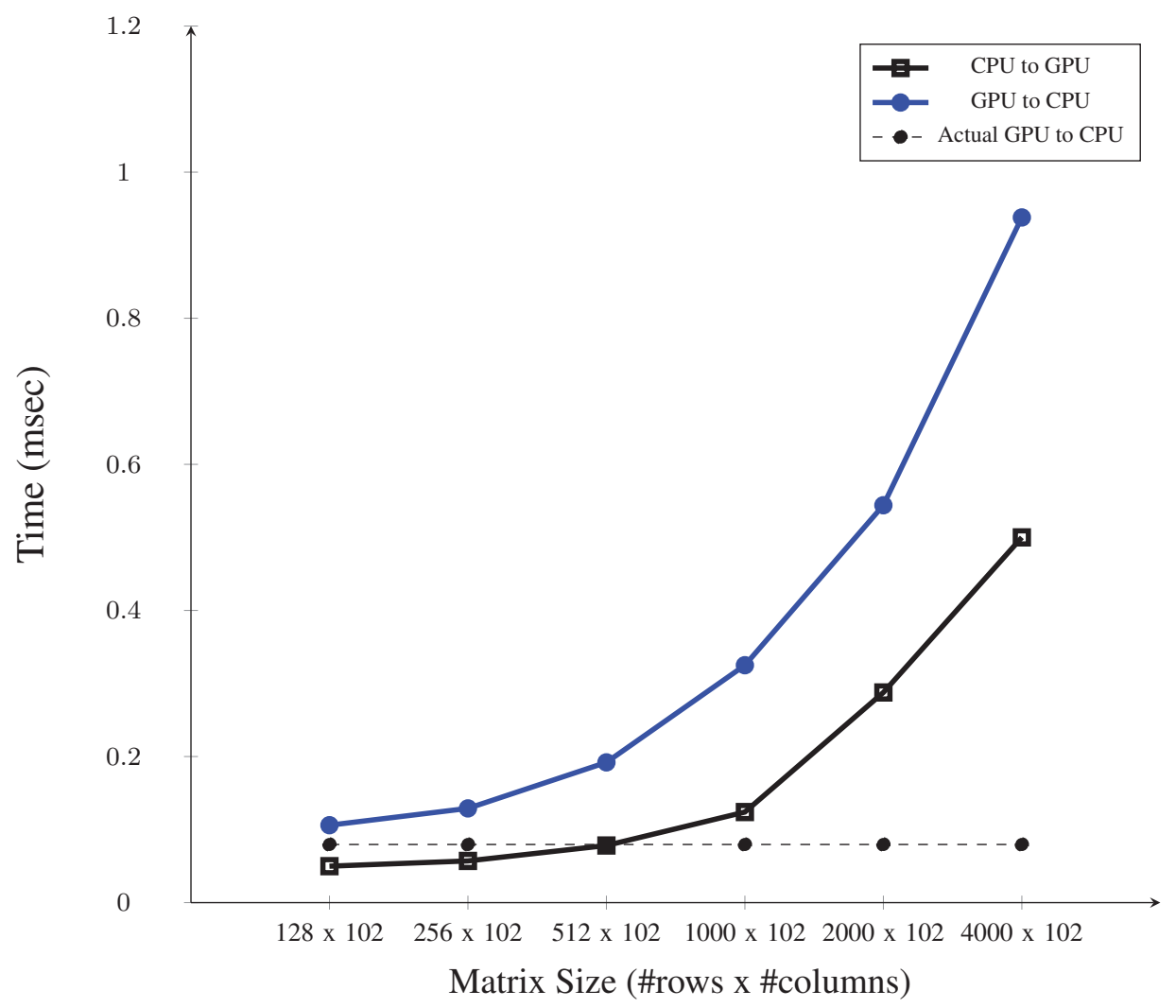

Figure 5.2: Transfer Time for Approach-1 for multiport VF

Figure 5.2 shows the data transfer times for varying matrix sizes. Here, $x$-axis represents the increasing matrix size and $y$-axis represents the corresponding time taken to transfer the data from CPU to GPU and GPU to CPU. Figure 5.2 also indicates the actual time taken during a single VF iteration for the GPU to CPU transfer time in Approach-1. It can be seen from the graph that,

(a) As the matrix size increases, the CPU to GPU transfer time gradually increases and vice versa. Also, it is evident from the graph that the GPU to CPU transfer time is much higher than CPU to GPU transfer time. This 
is because, when data is transferred from the GPU to CPU, memory synchronization is required, which adds to the excess time for GPU to CPU transfer.

(b) Note that the major data transfer requirement in the Approach-1 is transferring $\boldsymbol{A}_{l}$ matrices (3.26) in each iteration from the CPU to the GPU and transferring the collected residues (c) of the scaling function $\boldsymbol{\sigma}(s)$ from GPU to CPU. The GPU to CPU transfer is needed only in the final iteration of the parallel loop for sending the computed residues (c) to the CPU, size of which is very small $(N \times 1)$. Therefore, the time taken to transfer back the residues (c) from GPU to CPU is negligible and is represented using the dashed black line in Figure 5.2.

The numerical demonstration of the above times is given using the following example. Consider a system with 60 ports, 1000 frequency samples $(K)$ and 50 poles $(N)$ (25 complex poles and its 25 complex conjugate poles). Such a scenario leads to each $\boldsymbol{A}_{l}$ with a matrix size in (3.26) of $(2000 \times 102)$. Each $\boldsymbol{A}_{l}$ matrix needs to be transferred to GPU for QR factorization. Corresponding measured time taken to transfer a single $\boldsymbol{A}_{l}$ matrix in a particular parallel loop of VF iterations is 0.2877 msec. Since all the $\boldsymbol{A}_{l}$ matrices needs to be transferred in each VF iteration to the GPU in Approach-1, it leads to significant increase in data transfer time as well as the overall run time for VF.

The actual data being transferred back from GPU to CPU each iteration is the residues (c) of the scaling function $\boldsymbol{\sigma}(s)$ for which the transfer time is very minimal $(0.0796 \mathrm{~ms})$. 
Table 5.1 provides the data transfer times (CPU $\Leftrightarrow$ GPU) for different cases of matrix sizes. Column 3 shows the CPU time required for one Pole Refinement (henceforth referred to as PR in this thesis) iteration, in which $P^{2}$ number of $\boldsymbol{A}_{l}$ elements needs to be transferred from CPU to GPU. Column 4 shows the time for $12 \mathrm{PR}$ iterations assuming the case that 12 iterations were required for the pole convergence. Note that the $5^{\text {th }}$ column in the table (data transfer time from GPU to $\mathrm{CPU}$ ) is for information only, it is not used in Approach-1. On the other hand, the data relevant to VF using Approach-1 is the $7^{\text {th }}$ column in the table (actual GPU to CPU data tranfer time) as only the residues of the scaling function are written back and not the matrices.

\begin{tabular}{|c|c|c|c|c|c|c|}
\hline \multirow[b]{2}{*}{ Matrix size } & \multicolumn{3}{|c|}{ CPU to GPU } & \multirow[b]{2}{*}{$\begin{array}{c}\text { GPU to } \\
\text { CPU (For } \\
\text { one element } \\
\left.A_{l}\right)(\text { msec) }\end{array}$} & \multicolumn{2}{|c|}{$\begin{array}{c}\text { Actual GPU to CPU (for } \\
\text { transferring residue vector }(c) \text { ) }\end{array}$} \\
\hline & $\begin{array}{l}\text { For one } \\
\text { element } \\
A_{l}(\mathrm{msec})\end{array}$ & $\begin{array}{l}\text { For one PR } \\
\text { Iteration }\left(P^{2}=\right. \\
60 * 60= \\
3600 \text { elements }) \\
(\text { msec })\end{array}$ & $\begin{array}{l}\text { For 12 PR } \\
\text { iterations } \\
\text { (msec) }\end{array}$ & & $\begin{array}{l}\text { For one } \\
\text { PR } \\
\text { Iteration } \\
\text { (msec) }\end{array}$ & $\begin{array}{l}\text { For } 12 \text { PR } \\
\text { iterations (msec) }\end{array}$ \\
\hline $128 \times 102$ & 0.0499 & 179.6400 & 2155.6800 & 0.1057 & 0.0796 & 0.9552 \\
\hline $256 \times 102$ & 0.0571 & 205.5600 & 2466.7200 & 0.1286 & 0.0796 & 0.9552 \\
\hline $512 \times 102$ & 0.0782 & 281.4210 & 3377.0520 & 0.1917 & 0.0796 & 0.9552 \\
\hline $1000 \times 102$ & 0.1243 & 447.4800 & 5369.7600 & 0.3249 & 0.0796 & 0.9552 \\
\hline $2000 \times 102$ & 0.2877 & 1035.7200 & 12428.6400 & 0.5437 & 0.0796 & 0.9552 \\
\hline $4000 \times 102$ & 0.5000 & 1800.0000 & 216000.00 & 0.9382 & 0.0796 & 0.9552 \\
\hline
\end{tabular}

Table 5.1: Data transfer time for Approach-1 for multiport VF 


\subsubsection{Discussion on collection time for $\boldsymbol{R}_{l}^{22}$ matrices for residue vector (c) evaluation for Approach-1}

In this section, time taken to collect $\boldsymbol{R}_{l}^{22}$ from $\boldsymbol{R}_{l}$ in a CPU as well as in a GPU is investigated.

Following the QR factorization in GPU, $\boldsymbol{R}_{l}$ is stored in the GPU global memory. Next, $\boldsymbol{R}_{l}^{22}$ needs to be collected for the residue evaluation of the scaling function $\boldsymbol{\sigma}(s)$. This requires accessing the $\boldsymbol{R}_{l}$ that is stored in the GPU global memory and subsequently, writing $\boldsymbol{R}_{l}^{22}$ in the GPU global memory. For the previously defined system with 60 ports $(P), 1000$ frequency samples $(K)$ and 50 poles $(N)$, the time taken to collect all $\boldsymbol{R}_{l}^{22}$ matrices in a single iteration of VF is $0.442 \mathrm{msec}$.

\subsubsection{Discussion on QR factorization time for Approach-1}

In this subsection, a discussion comparing the computational performance of the GPU and CPU platforms for QR factorization is given. 


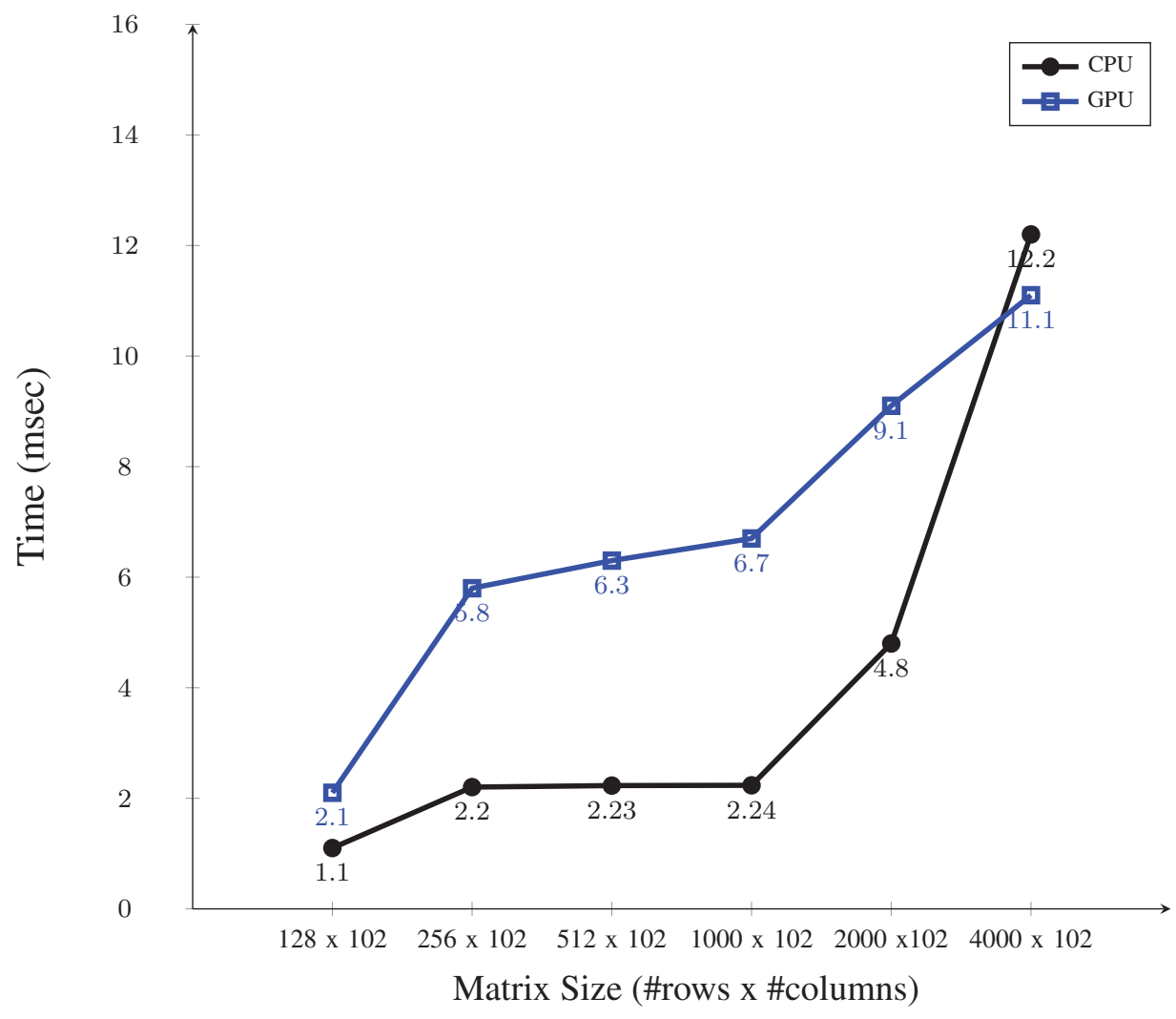

Figure 5.3: Comparison of time for QR factorization for one $\boldsymbol{A}_{l}$ element in GPU and CPU

Figure 5.3 shows the computational time for QR factorization for varying matrix sizes of one $\boldsymbol{A}_{l}$ element. Here, $x$-axis represents the increasing matrix size and $y$-axis represents the time taken to execute a single $\mathrm{QR}$ factorization by a single CPU and a single GPU. It can be seen from the Figure 5.3 that,

(a) As the matrix size increases, the time taken to perform QR increases for both the GPU and CPU. Also, the Figure 5.3 indicates that the time spent on QR factorization by GPU is much higher than CPU till the matrix of 
size $(4000 \times 102)$. However, for matrices with size greater than or equal to $(4000 \times 102)$, GPU takes lower time than CPU. This is the peak achievable point when GPU can be utilized efficiently.

For the purpose of practical insight, consider the previously used system with 60 ports $(P), 1000$ frequency points $(K)$ and 50 poles $(N)$ which results in an $\boldsymbol{A}_{l}$ element of size $(2000 \times 102)$. A numerical demonstration of the above discussion is given in the following.

As seen in Figure 5.3, for this matrix size, a single CPU performs QR factorization (in $4.8 \mathrm{msec}$ ) faster than a single GPU (in $9.8 \mathrm{msec}$ ). Hence the matrix size becomes an important factor to consider for obtaining optimal efficiency while allocating the tasks and loads to CPUs and GPUs. 


\subsubsection{Performance comparison of Approach-1 with multiple CPUs and a sin- gle GPU versus just the multiple CPUs}

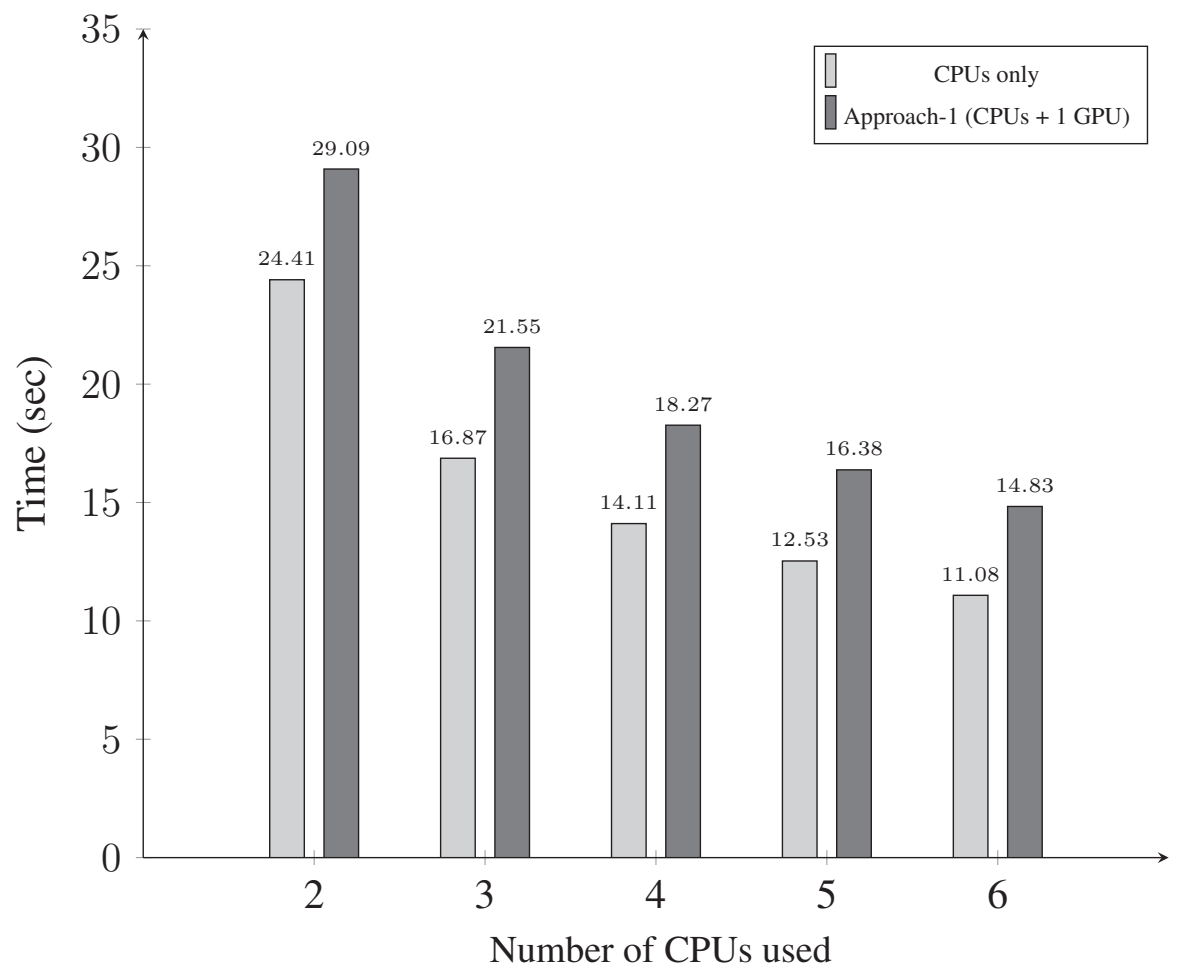

Figure 5.4: Comparison of Approach-1 with multiple CPUs and a single GPU versus just the CPUs

Figure 5.4 shows the performance comparison of Approach-1 with multiple CPUs and a single GPU versus just the CPUs for one PR iteration of VF is given for the previously defined system with 60 ports $(P), 1000$ frequency samples $(K)$ and 50 poles $(N)$. Here, $x$-axis represents the number of CPUs used and $y$-axis represents the time taken to execute a single PR iteration of VF. It can be seen from Figure 5.4 that, Approach-1 does not lead to efficiency gain for the matrix size under consideration. This is mainly because 
1. Depending on the size of the $\boldsymbol{A}_{l}$, the time taken for $\mathrm{QR}$ factorization of a single $\boldsymbol{A}_{l}$ matrix in a single GPU can be higher than the time taken in a single CPU (as seen in Figure 5.3).

2. The data transfer time from CPU to GPU is excessive which needs to be minimized as each $\boldsymbol{A}_{l}$ needs to be sent to GPU one at a time (see Figure 5.2).

3. Also, CPUs remain idle while they wait for GPU to finish QR factorization of $\boldsymbol{A}_{l}$ matrices in the parallelization loop and also during the computation of residue vector in every pole refinement iteration of VF.

Next, Approach-1 will be further explored using two GPUs along with multiple CPUs for parallel VF execution.

\subsubsection{Approach-1 with Multiple CPUs and two GPUs}

In this subsection, performance of Approach-1 is evaluated in the presence of two GPUs and multiple CPUs. Parallel execution steps for the proposed Approach-1 with Multiple CPUs and 2GPUs are summarized below:

(a) The formulation of $\boldsymbol{A}_{l}$ matrices is done as in step (a) of section 5.1.1.

(b) Once the $\boldsymbol{A}_{l}$ matrix is available, it is sent to any of the available GPUs for immediate QR factorization to obtain $\boldsymbol{Q}_{l}$ and $\boldsymbol{R}_{l}$ (i.e., QR factorization of $\boldsymbol{A}_{l}$ matrices done in parallel in GPUs).

(c) Next, the $\boldsymbol{R}_{l}^{22}$ and $\boldsymbol{Q}_{l}^{b}$ matrices are collected for each of the $\boldsymbol{A}_{l}$ matrices 
in one of the GPUs and the residues (c) for the scaling function $\boldsymbol{\sigma}(s)$ are computed in that same GPU, using (3.18).

\subsubsection{Performance of Approach-1 with 2-GPUs and multiple CPUs}

In this subsection, a discussion comparing the performance of Approach-1 with Multiple CPUs and two GPUs, Approach-1 with Multiple CPUs and one GPU and CPUs alone for the None Splitting Strategy is given for the same previous system with 60 ports $(P), 1000$ frequency samples $(K)$ and 50 poles $(N)$ for one $\mathrm{PR}$ iteration of VF is given.

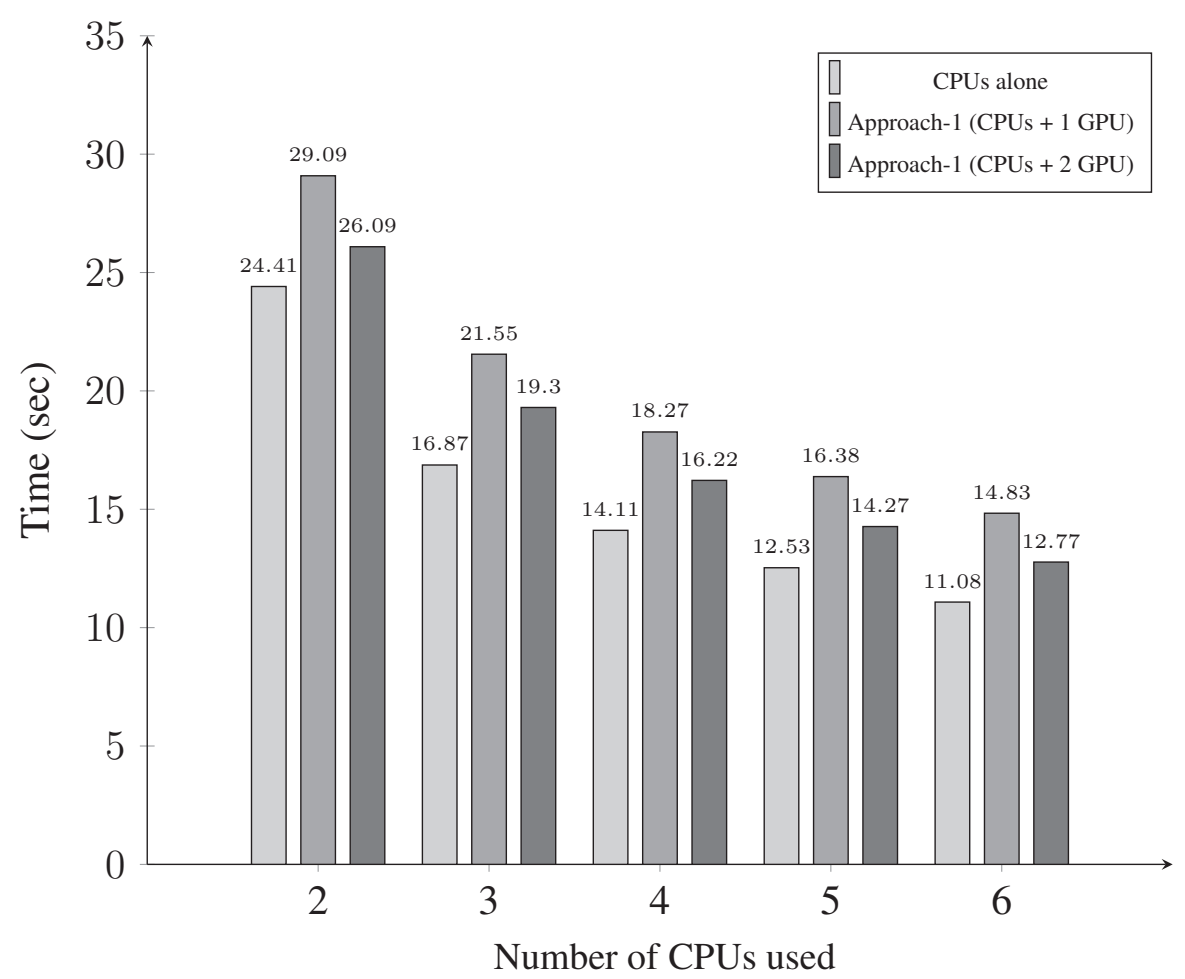

Figure 5.5: Performance comparison of Approach-1 with multiple CPUs and a single GPU, Approach-1 with multiple CPUs and two GPUs with CPUs alone. 
Figure 5.5 shows the total computational times for one PR iteration of VF while using Approach-1 with multiple CPUs and a single GPU, Approach-1 with multiple CPUs and two GPUs, as well as the case with just the CPUs. Here, x-axis represents the number of CPUs used and y-axis represents the time taken to execute a single PR iteration of VF.

As can be seen from the Figure 5.5, using two GPUs with multiple CPUs brings slightly better performance than using one GPU with multiple CPUs. This is because while using two GPUs, QR factorization is done parallely in both of the GPUs. It can also be seen from the Figure 5.5, that even while using two GPUs in this approach does not lead to efficiency gain compared to using just the CPUs. This is due to the fact that the QR computational time taken by CPU for a single $\boldsymbol{A}_{l}$ matrix is much lower when compared with the QR computational time in GPU. 


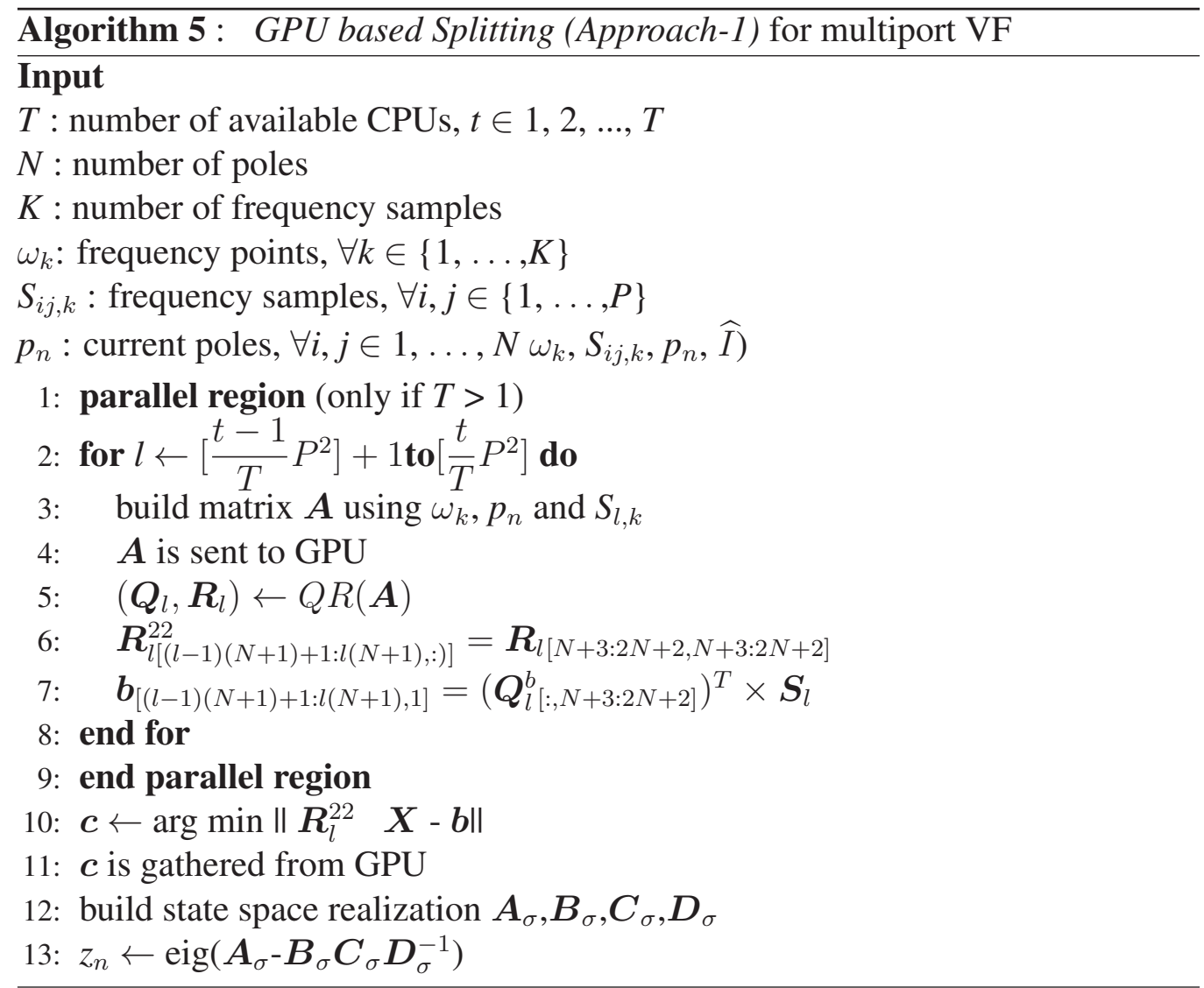

\subsection{Approach-2: Transfer of block of $A_{l}$ matrices from CPU to GPU and parallel QR factorization in CPUs and GPU (single scaling function)}

In this splitting strategy, formulation of $\boldsymbol{A}_{l}$ matrix (3.26) for a total of $P^{2} \boldsymbol{S}_{i j}$ elements is done independently in separate CPUs. All the $\boldsymbol{A}_{l}$ elements are grouped into two blocks, with $n_{g}$ number of $\boldsymbol{A}_{l}$ elements for QR factorization in GPU and $n_{c}$ number of $\boldsymbol{A}_{l}$ elements for QR factorization in CPUs. The numbers $n_{g}$ and $n_{c}$ are decided in a way that both the CPUs and GPU are kept busy in the parallel loop of a PR iteration. As soon as $\mathrm{n}_{g}$ number of $\boldsymbol{A}_{l}$ matrices are parallely 
formulated in CPUs, they are sent at once to the GPU for QR factorization. The CPUs continue to parallely formulate the remaining $n_{c} \boldsymbol{A}_{l}$ matrices and also their QR factorization. The $\boldsymbol{R}_{l}^{22}$ and $\boldsymbol{Q}_{l}^{b}$ matrices are collected from the GPU and CPU parallely in the CPU DRAM memory associated with one of the CPUs (refer to Figure 4.2). This is followed by the residue (c) evaluation for the scaling function $\boldsymbol{\sigma}(s)$ in the same CPU. At the end of the final PR iteration, a common set of poles for the entire macromodel is obtained.

Parallel execution steps for the proposed Approach-2 are outlined below.

(a) Each $\boldsymbol{A}_{l}$ matrix (i.e., $\boldsymbol{A}_{1}, \boldsymbol{A}_{2}, \ldots, \boldsymbol{A}_{l}, \ldots, \boldsymbol{A}_{P^{2}}$ ) of (3.26) is formed independently in separate CPUs. Let ' $T$ ' be the total number of available CPUs. For parallel formulation of each $\boldsymbol{A}_{l}$, they are assigned as in (3.27). Based on the available number of CPUs, (3.27) parallel formulation is done in approximately $\frac{P^{2}}{T}$ loops. For computing the first $n_{g}$ number of $\boldsymbol{A}_{l}$ elements approximately $\frac{n_{g}}{T}$ loops are required.

(b) Once $n_{g}$ number of $\boldsymbol{A}_{l}$ matrices are obtained, they are stored as $\boldsymbol{A}_{G P U}$ $\leftarrow \boldsymbol{A}_{1}, \boldsymbol{A}_{2}, \ldots, \boldsymbol{A}_{n g}$ and sent at once to a single GPU for parallel QR factorization to obtain $\left(\boldsymbol{Q}_{l}, \boldsymbol{R}_{l}\right)$ for each of the $\boldsymbol{A}_{l}$ matrices. This is followed by collecting the respective $\boldsymbol{R}_{l}^{22}$ and $\boldsymbol{Q}_{l}^{b}$ for all $\boldsymbol{A}_{l}$ matrices and sending them back to a CPU at once.

(c) While the step (b) is in progress, CPUs continue with parallel formulation and QR factorization of the remaning $n_{c} \boldsymbol{A}_{l}$ matrices and the respective $\boldsymbol{R}_{l}^{22}$ and $\boldsymbol{Q}_{l}^{b}$ matrices are stored in the DRAM associated with one of the CPUs. The step (b) and step (c) are executed in parallel until the $\boldsymbol{Q}_{l}$ and $\boldsymbol{R}_{l}$ of all 
the $\boldsymbol{A}_{l}$ matrices are obtained $\left(n_{g}\right.$ and $n_{c}$ are selected such that CPUs and GPU are kept busy throughout the parallel loop).

(d) Once the $\boldsymbol{R}_{l}^{22}$ and $\boldsymbol{Q}_{l}^{b}$ matrices are collected for each of $\boldsymbol{A}_{l}$ matrices from the GPU and the CPUs in one of the available CPUs, the residue vector (c) for the scaling function $\boldsymbol{\sigma}(s)$ is computed in that same CPU itself, using (3.18).

For the purpose of illustrating the above approach, consider the previously defined 4-port system with scattering matrix, $S$, with individual elements that are represented as in (3.28). Let the available number of CPUs be 4 and GPU be 1. The solution process for the Approach-2 (i.e., Approach-2 with multiple CPUs and a single $G P U$ ) for this example is illustrated in Figure 5.6. As seen, formulation of individual matrices $\boldsymbol{A}_{1}, \boldsymbol{A}_{2}, \ldots, \boldsymbol{A}_{l} \ldots, \boldsymbol{A}_{n_{g}}$ are done parallely in CPUs. The QR factorization is parallely done in GPU and CPUs. The $\boldsymbol{R}_{l}^{22}$ and $\boldsymbol{Q}_{l}^{b}$ matrices are collected from the GPU and CPU parallely in one of the available CPUs. At the end, the residue computation for the scaling function $\boldsymbol{\sigma}(s)$ for the entire $\boldsymbol{S}$ matrix is done in one of the available CPUs. The pseudocode corresponding to the Approach-2 is given in Algorithm 5. 


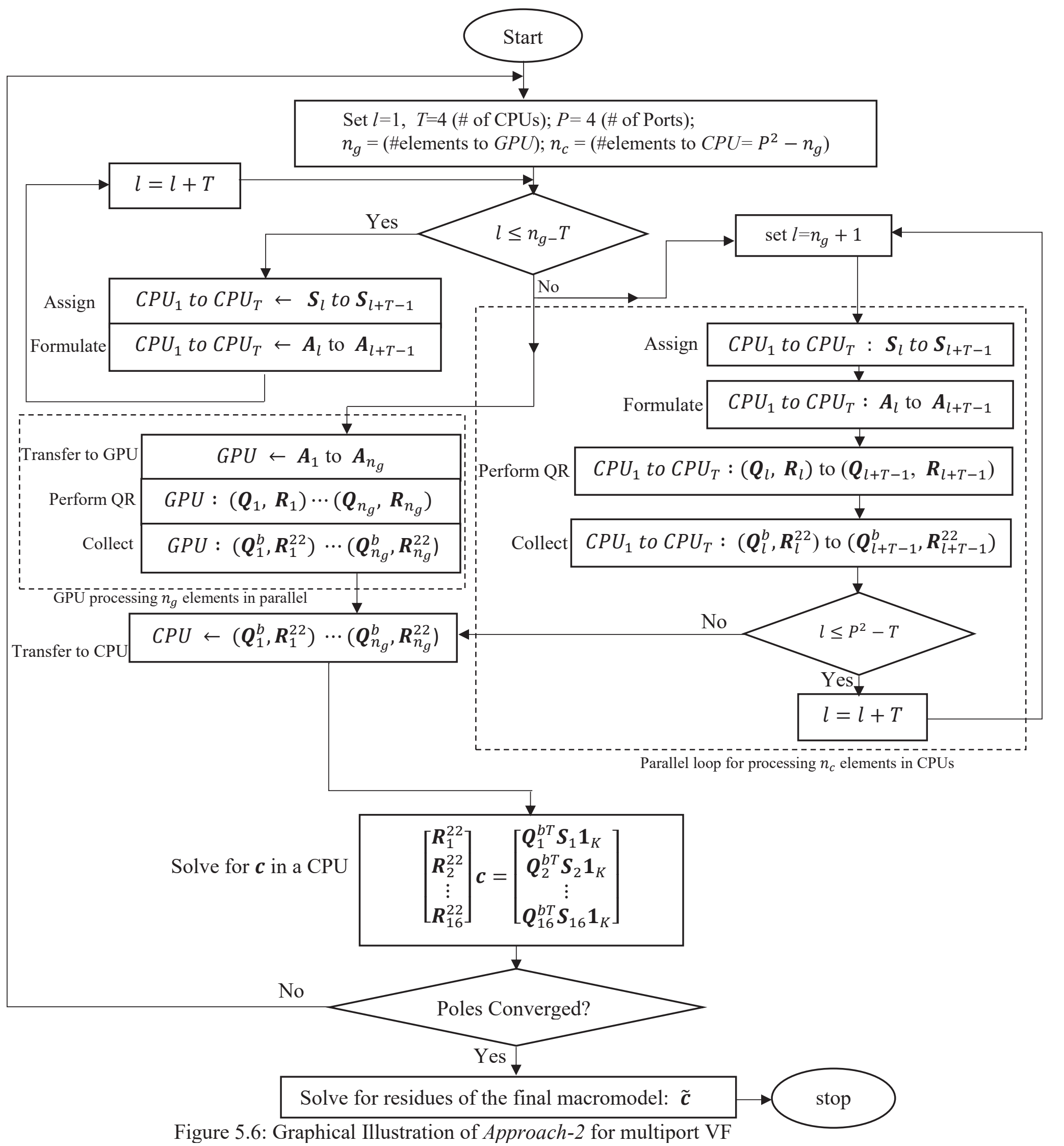




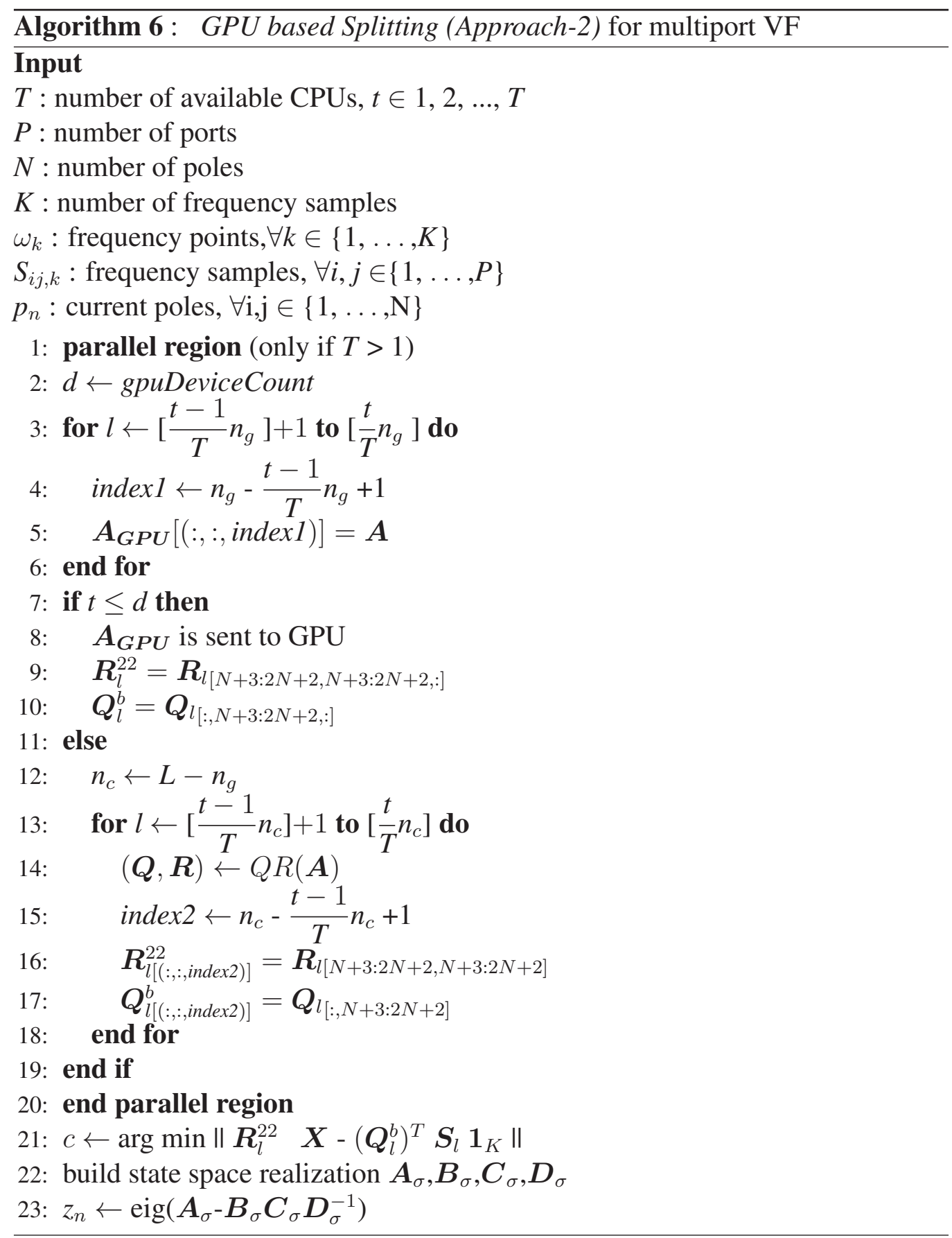




\subsubsection{Performance Comparison of Approach-2 (with multiple CPUs and one GPU) and just the multiple CPUs}

In this subsection, a discussion comparing the performance of Approach-2 (with multiple CPUs and one GPU) and just the multiple CPUs is given. The results are discussed with respect to the previously considered system with 60 ports $(P)$, 1000 frequency samples $(K)$ and 50 poles $(N)$.

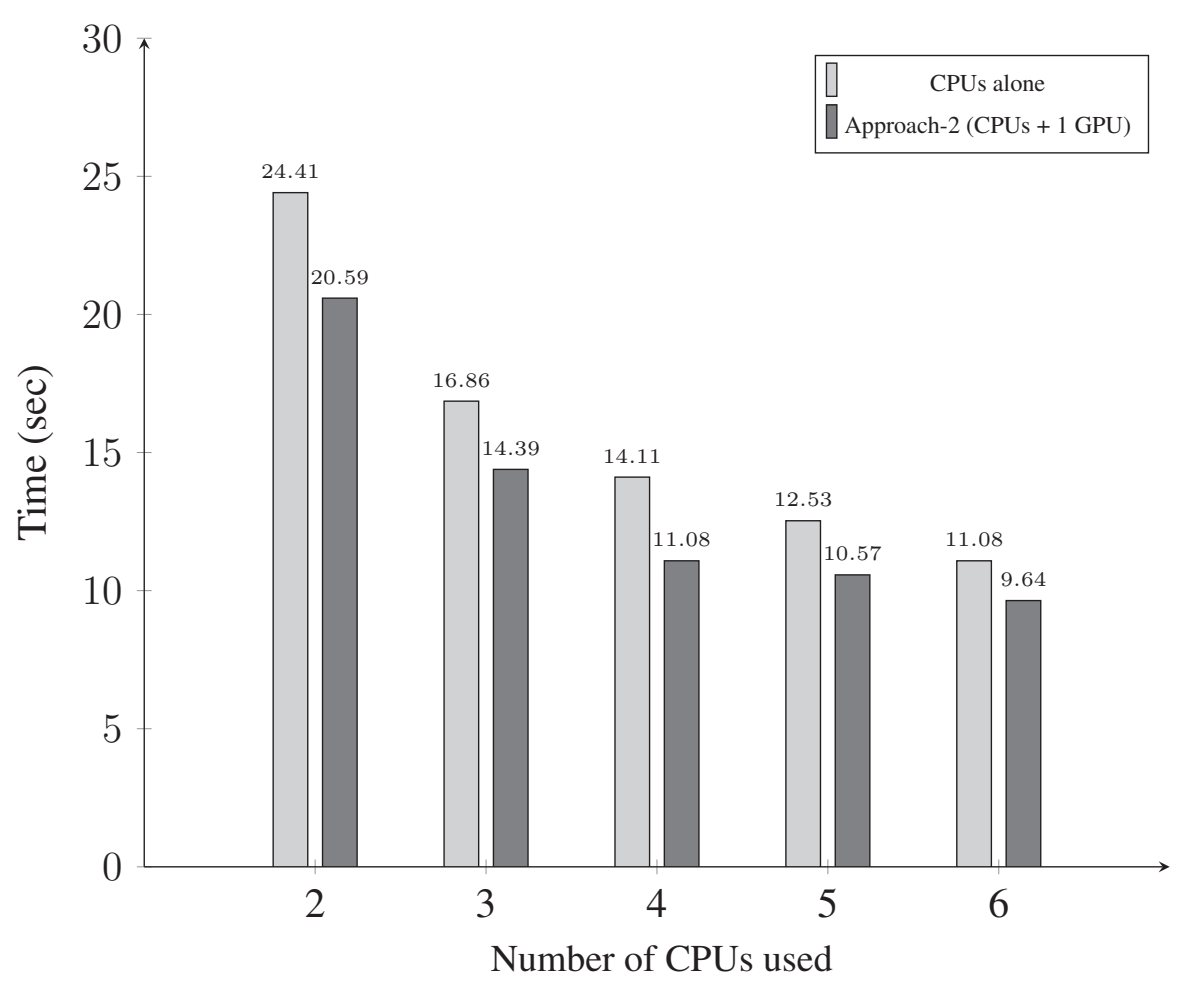

Figure 5.7: Performance Comparison of Approach-2 and just the Multiple CPUs

Figure 5.7 shows the comparison of the computational performance of both the approaches. Here, $x$-axis represents the number of CPUs used and $y$-axis represents the time taken to execute a single PR iteration of VF. It can be seen from the 
graph that Approach-2 brings better performance compared to the case of using just the multiple CPUs. This is because of the following reasons:

(a) In Approach-2, when a GPU was performing QR factorization for $n_{g}$ number of $\boldsymbol{A}_{l}$ matrices, all the CPUs are also parallely performing QR factorization of the remaining $n_{c} \boldsymbol{A}_{l}$ matrices. Using multiple CPUs and a GPU parallely this way enables the GPU and all the CPUs to be busy in the parallel loop which increases the efficiency. This overcomes one of the major bottlenecks in Approach-1, which is CPUs remaining idle when a GPU is sequentially performing $\mathrm{QR}$ factorization. Additional minor savings are achieved during the copy of $n_{g} \boldsymbol{A}_{l}$ elements from CPU to GPU as the copying is initiated at the same time.

(b) Another data transfer cost in the Approach-2, is transferring $\boldsymbol{R}_{l}^{22}, \boldsymbol{Q}_{l}^{b}$ matrices for $n_{g}$ number of $\boldsymbol{A}_{l}$ elements at the same time from GPU to a single CPU. However, this data transfer overlaps during the period when multiple CPUs are busy computing their part of the QR and transferring the corresponding $\boldsymbol{R}_{l}, \boldsymbol{Q}_{l}$ matrices to one of the available CPUs.

(c) Even though, $Q R$ factorization for a single $\boldsymbol{A}_{l}$ matrix of size $(2000 \times 102)$ is faster in a CPU than in a GPU (please refer to Section 5.1.4), it is addressed in Approach-2 by using GPU in parallel with multiple CPUs to perform QR factorization, to provide a overall better performance when compared to the case of using just the CPUs. For a proper load balancing in this example, $n_{g}$ and $n_{c}$ are selected as in Table 5.2

Minor bottleneck of this approach is that GPU and the remaining CPUs remain 
idle when one of the CPUs is evaluating residues (c) of the scaling function $(\boldsymbol{\sigma}(s))$ at the final step of a PR iteration.

\begin{tabular}{|l|l|l|l|l|}
\hline \multicolumn{6}{|c|}{ For one PR iteration $\left(\boldsymbol{P}^{\mathbf{2}}=\mathbf{6 0} * \mathbf{6 0}=\mathbf{3 6 0 0}\right.$ elements $)$} \\
\hline $\begin{array}{l}\text { Number of } \\
\begin{array}{l}\text { CPUs used } \\
\text { in parallel }\end{array}\end{array}$ & $\begin{array}{l}\text { Number of } \boldsymbol{A}_{\boldsymbol{l}} \text { elements sent to } \\
\text { GPU }\end{array}$ & $\begin{array}{l}\text { Number of } \boldsymbol{A}_{\boldsymbol{l}} \text { elements sent to } \\
\text { CPUs }\end{array}$ \\
\cline { 2 - 6 } & \multicolumn{2}{|c|}{$\boldsymbol{n}_{\boldsymbol{g}}$} & $\frac{\boldsymbol{n}_{\boldsymbol{g}}}{\boldsymbol{P}^{\mathbf{2}}} \mathbf{X} \mathbf{1 0 0}$ & \multicolumn{2}{c|}{$\boldsymbol{n}_{\boldsymbol{c}}$} & $\frac{\boldsymbol{n}_{\boldsymbol{C}}}{\boldsymbol{P}^{\mathbf{2}}} \mathbf{X} \mathbf{1 0 0}$ \\
\hline 2 & 2160 & $60 \%$ & 1440 & $30 \%$ \\
\hline 3 & 1620 & $44 \%$ & 1980 & $56 \%$ \\
\hline 4 & 1224 & $34 \%$ & 2376 & $66 \%$ \\
\hline 5 & 1008 & $28 \%$ & 2592 & $72 \%$ \\
\hline 6 & 828 & $23 \%$ & 2772 & $77 \%$ \\
\hline
\end{tabular}

Table 5.2: $n_{g}$ and $n_{c}$ values for Approach-2 for multiport VF

\subsection{Approach-3: Transfer of block of $A_{l}$ matrices from CPUs to GPU, par- allel QR factorization and residue evaluation in CPUs as well as in GPU (individual scaling functions)}

The main difference in this strategy and the Approach-2 is that each element $\boldsymbol{S}_{i j}$ will have its own scaling function, resulting in its own set of poles. Instead of sending back $\boldsymbol{R}_{l}^{22}$ and $\boldsymbol{Q}_{l}^{b}$ to CPU, GPU itself will compute the corresponding residue $\left(\boldsymbol{c}_{l}\right)$ for the scaling function $\left(\boldsymbol{\sigma}_{l}(s)\right)$, thus minimizing the data transfer cost from GPU to CPU.

In this splitting strategy, the formulation of $\boldsymbol{A}_{l}$ matrix (3.26) for a total of $P^{2}$ $S_{i j}$ elements is done independently in separate CPUs. All the $\boldsymbol{A}_{l}$ elements are grouped into two blocks, with $n_{g}$ number of $\boldsymbol{A}_{l}$ elements for $\mathrm{QR}$ factorization as 
well as corresponding residue evaluation in GPU and $n_{c}$ number of $\boldsymbol{A}_{l}$ elements for QR factorization as well as corresponding residue evaluation in CPUs. The numbers $n_{g}$ and $n_{c}$ are decided in a way that both the CPUs and GPU are kept busy in the parallel loop of PR iteration. As soon as $n_{g}$ number of $\boldsymbol{A}_{l}$ matrices are parallely formulated in CPUs, they are sent along with the corresponding $n_{g}$ number of $\boldsymbol{S}_{l}$ vectors of size $(2 K \times 1)$ (note that, these $\boldsymbol{S}_{l}$ vectors are needed for residue evaluation and they are sent at the beginning along with $\boldsymbol{A}_{l}$ matrices) to the GPU for QR factorization. After the QR factorization, for these $n_{g}$ elements, residues $\boldsymbol{c}_{l}$ of the scaling function $\boldsymbol{\sigma}_{l}(s)$ (which is specific to the corresponding $\boldsymbol{A}_{l}$ in this approach) are also evaluated in parallel in GPU. The CPUs continue with parallely formulating the remaining $n_{c} \boldsymbol{A}_{l}$ matrices, computing $\mathrm{QR}$ factorization and evaluating the residues $\left(\boldsymbol{c}_{l}\right)$ of the corresponding scaling functions $\boldsymbol{\sigma}_{l}(s)$. At the end of the converged PR iteration, each $S_{i j}$ element will have its own set of poles.

Parallel execution steps for the proposed Approach-3 are outlined below.

(a) Each $\boldsymbol{A}_{l}$ matrix (i.e., $\boldsymbol{A}_{1}, \boldsymbol{A}_{2}, \ldots, \boldsymbol{A}_{l}, \ldots, \boldsymbol{A}_{P^{2}}$ ) of (3.26) is formed independently in separate CPUs. Let ' $T$ ' be the total number of available CPUs. For parallel formulation of each $\boldsymbol{A}_{l}$, they are assigned as in (3.27). Based on the available number of CPUs, (3.27) parallel formulation is done in approximately $\frac{P^{2}}{T}$ loops. For computing the first $n_{g}$ number of $\boldsymbol{A}_{l}$ elements approximately $\frac{n_{g}}{T}$ loops are required.

(b) Once $n_{g}$ number of $\boldsymbol{A}_{l}$ matrices are obtained, they are stored as $\boldsymbol{A}_{G P U} \leftarrow$ $\boldsymbol{A}_{1}, \boldsymbol{A}_{2}, \ldots, \boldsymbol{A}_{n_{g}}$ and it is sent along with $\boldsymbol{b}_{G P U} \leftarrow \boldsymbol{S}_{1}, \boldsymbol{S}_{2}, \ldots, \boldsymbol{S}_{n_{g}}$, at once to the GPU. GPU performs parallel QR factorization to obtain $\left(\boldsymbol{Q}_{l}, \boldsymbol{R}_{l}\right)$ for 
each of the $\boldsymbol{A}_{l}$ matrices. Subsequently, $\boldsymbol{R}_{l}^{22}$ and $\boldsymbol{Q}_{l}^{b}$ for each of the $n_{g} \boldsymbol{A}_{l}$ matrices are extracted and residues $\left(\boldsymbol{c}_{l}\right)$ are computed for the corresponding scaling function $\boldsymbol{\sigma}_{l}(s)$ (which is specific to that $\boldsymbol{S}_{i j}$ element) in the GPU itself, using the relation in (3.29).

(c) While step (b) is being processed, CPUs continue with parallel formulation and QR factorization of the remaining $n_{c} \boldsymbol{A}_{l}$ matrices. Next, the resulting $\boldsymbol{R}_{l}^{22}$ and $\boldsymbol{Q}_{l}^{b}$ matrices are collected and then the residues of the corresponding scaling function $\boldsymbol{\sigma}_{l}(s)$ (which is specific to that $\boldsymbol{S}_{i j}$ element) are also computed in the respective CPUs, using the relation in (3.29).

Since the step (b) and step (c) are executed in parallel in both the CPUs and GPU, they are kept busy throughout the parallel loop.

For the purpose of illustrating the above approach, consider a 4-port system with scattering matrix, $S$, with individual elements that are represented as in (3.28). Let the available number of CPUs be 4 and GPU be 1 . The solution process for the Approach-3 for this example is illustrated in Figure 5.8. As seen, formulation of individual matrices $\boldsymbol{A}_{1}, \boldsymbol{A}_{2}, \ldots, \boldsymbol{A}_{l} \ldots, \boldsymbol{A}_{P^{2}}$ are done parallely in CPU. The QR factorization and the residue evaluation for respective $\boldsymbol{A}_{l}$ matrices is parallely done in GPU and CPUs. At the end of the final iteration, individual pole sets as well as the residues of the scaling function $\boldsymbol{\sigma}(s)$ are computed for each of the $\boldsymbol{S}_{i j}$ element. The pseudocode corresponding to the Approach-3 is given in Algorithm 7. 


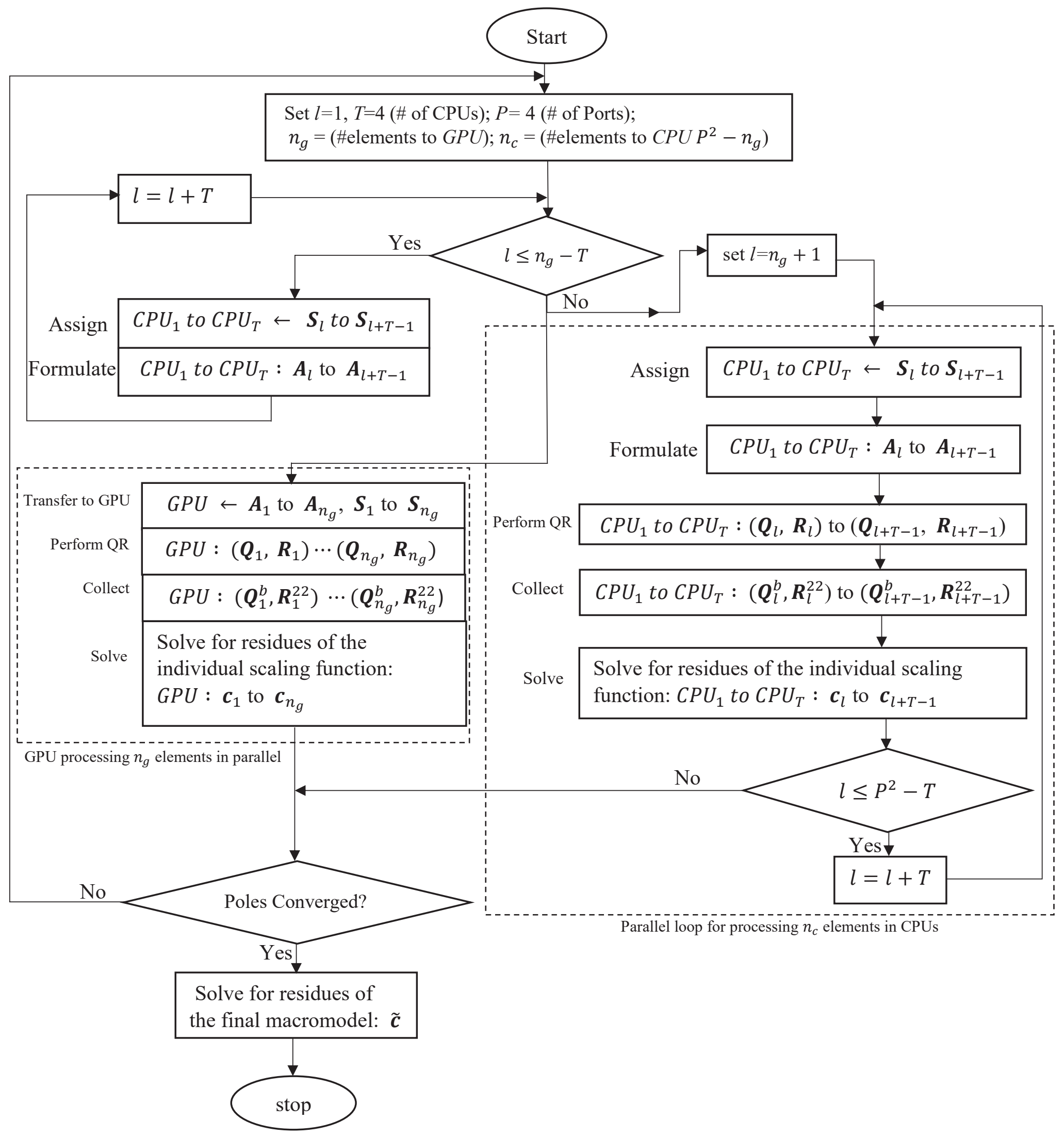

Figure 5.8: Graphical Illustration of Approach-3 for multiport VF 


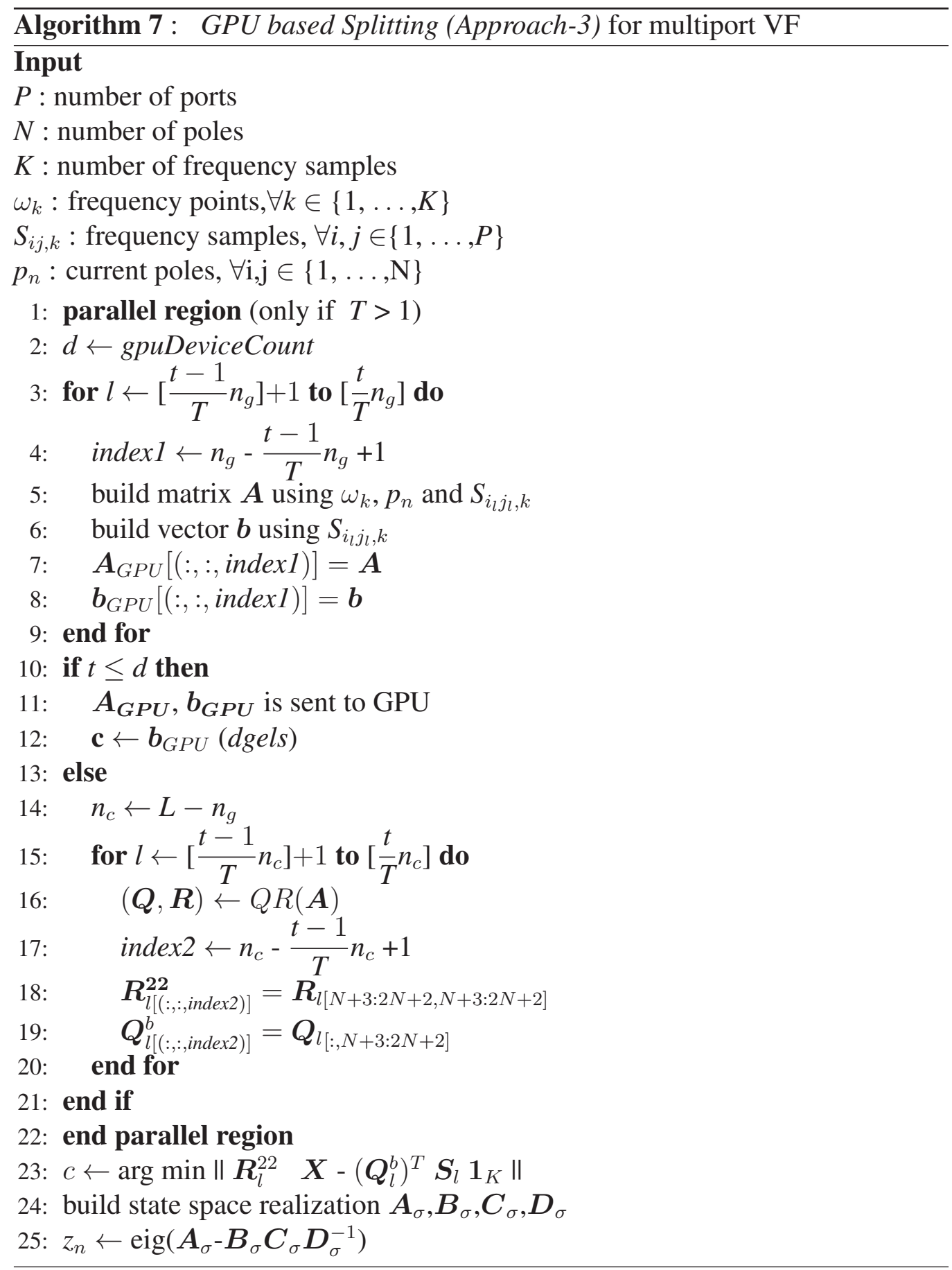




\subsubsection{Performance comparison of Approach-3 (with multiple CPUs and one GPU) and just the multiple CPUs (All Splitting)}

In this subsection, a discussion comparing the performance of Approach-3 (with multiple CPUs and one GPU) and just the CPUs for the All Splitting Strategy is given. The results are discussed with respect to previously defined system with 60 ports $(P), 1000$ frequency samples $(K)$ and 50 poles $(N)$ and one PR iteration of VF. Corresponding results are given in Figure 5.9.

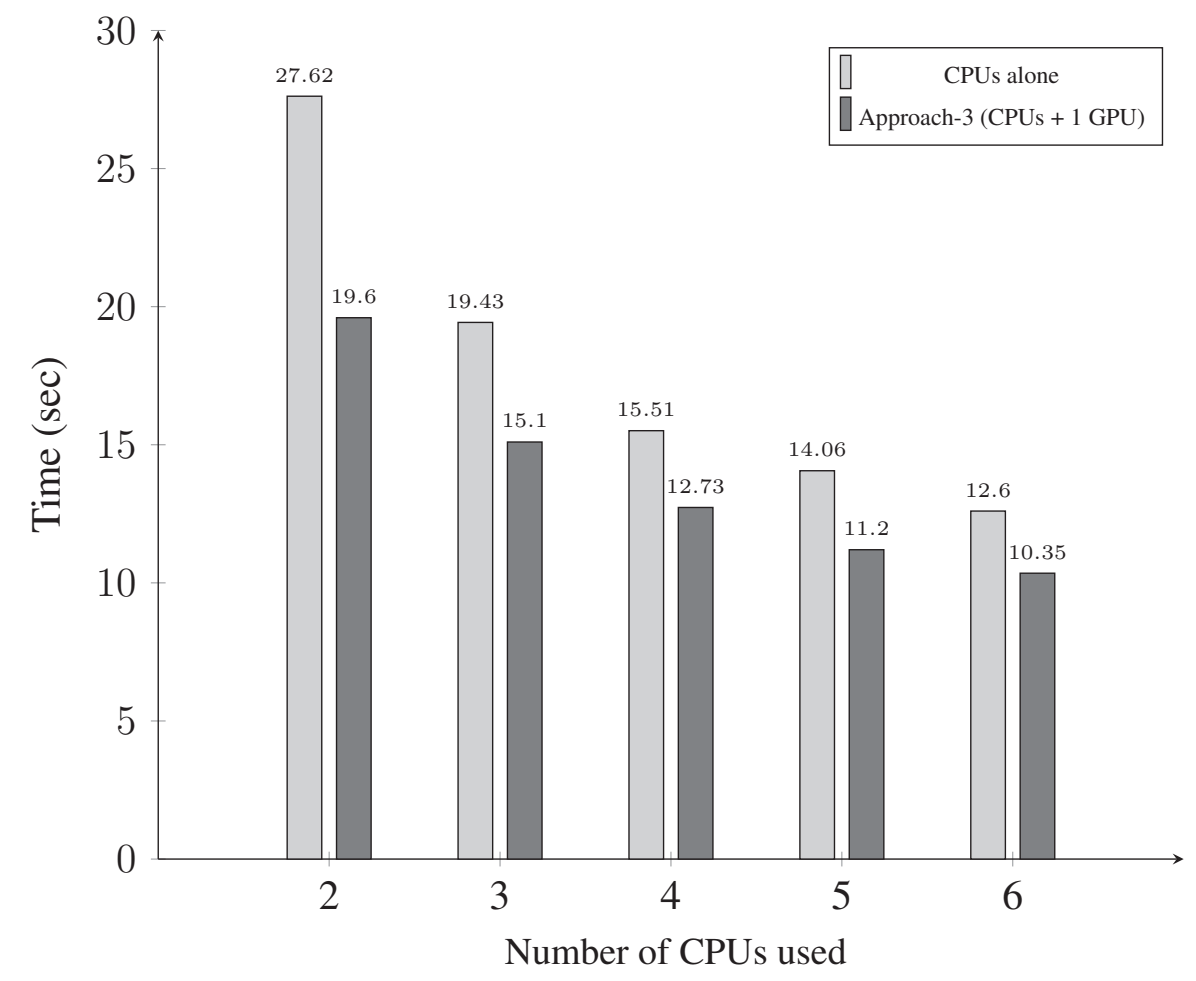

Figure 5.9: Performance Comparison of Approach-3 and just the Multiple CPUs for the All Splitting Strategy

Here, $x$-axis represents the number of CPUs used and $y$-axis represents the time 
taken to execute a single PR iteration of VF. It can be seen from the graph that Approach-3 brings better performance compared to the case of using just the multiple CPUs. This is because of the following reason:

In Approach-3, formulation, QR factorization as well as residue evaluation of all $\boldsymbol{A}_{l}$ matrices $\left(\boldsymbol{A}_{1}, \boldsymbol{A}_{2}, \ldots, \boldsymbol{A}_{P^{2}}\right)$ are done in parallel in a loop using the available number of CPUs and GPU. This overcomes the minor bottleneck in Approach-2, while evaluating residues (c) of the scaling function $(\boldsymbol{\sigma}(s))$ in one of the CPUs, the remaining CPUs and GPU are kept idle. Any delay in waiting for $\boldsymbol{R}_{l}^{22}, \boldsymbol{Q}_{l}^{b}$ is eliminated in this approach. Also, the additional data transfer time from CPU to GPU of $\boldsymbol{S}_{l}$ vector of size $(2 K \times 1)$ for $n_{g} \boldsymbol{A}_{l}$ elements is minimal and does not significantly affect the overall VF time. 


\section{Chapter 6}

\section{Computational Results}

This chapter presents computational results to validate the accuracy and efficiency of the proposed approaches for VF using mixed GPU and CPU platforms described in Chapter 5. For this purpose, three examples are presented. The multiport S-parameter tabulated data in these examples corresponds to that of diverse high-speed modules such as multiconductor interconnects and IC packages. The first example presents a smaller case of 31-port network. The second example considers a medium case of 60-port network and the third example considers a larger case of 120-port network.

The performance comparison between the GPU based approaches and just the CPUs is provided here for one PR iteration of VF. Also, in order to obtain meaningful statistical results, each run is repeated five times and average mean time is recorded. Also rms error $=5 \times 10^{-4}$, is used for accuracy convergence between the proposed model and original data.

All the tests are performed and evaluated using the Compute Canada platform (a national computing grid) [24], which has the module configuration of four CPU cores (Intel Xeon E5-2650), 64-GB RAM, and a GPU. The GPU (Pascal P100) consist of 3584 cores, $4096 \mathrm{~KB}$ of L2 cache memory, and 12-GB RAM and oper- 
ates at $1.328 \mathrm{GHz}$ clock frequency.

\subsection{Example 1 : 30 port network}

In this example, the performance comparison of Approach-1 (multiple CPUs and a single GPU), Approach-2 and Approach-3 versus the case of just the multiple CPUs is given for a system with 30 port $(P), 26$ poles $(N)(13$ complex poles, 13 complex conjugate poles) and 591 frequency samples $(K)$. Note that corresponding size of each $\boldsymbol{A}_{l}$ matrix is $(2000 \times 54)$.

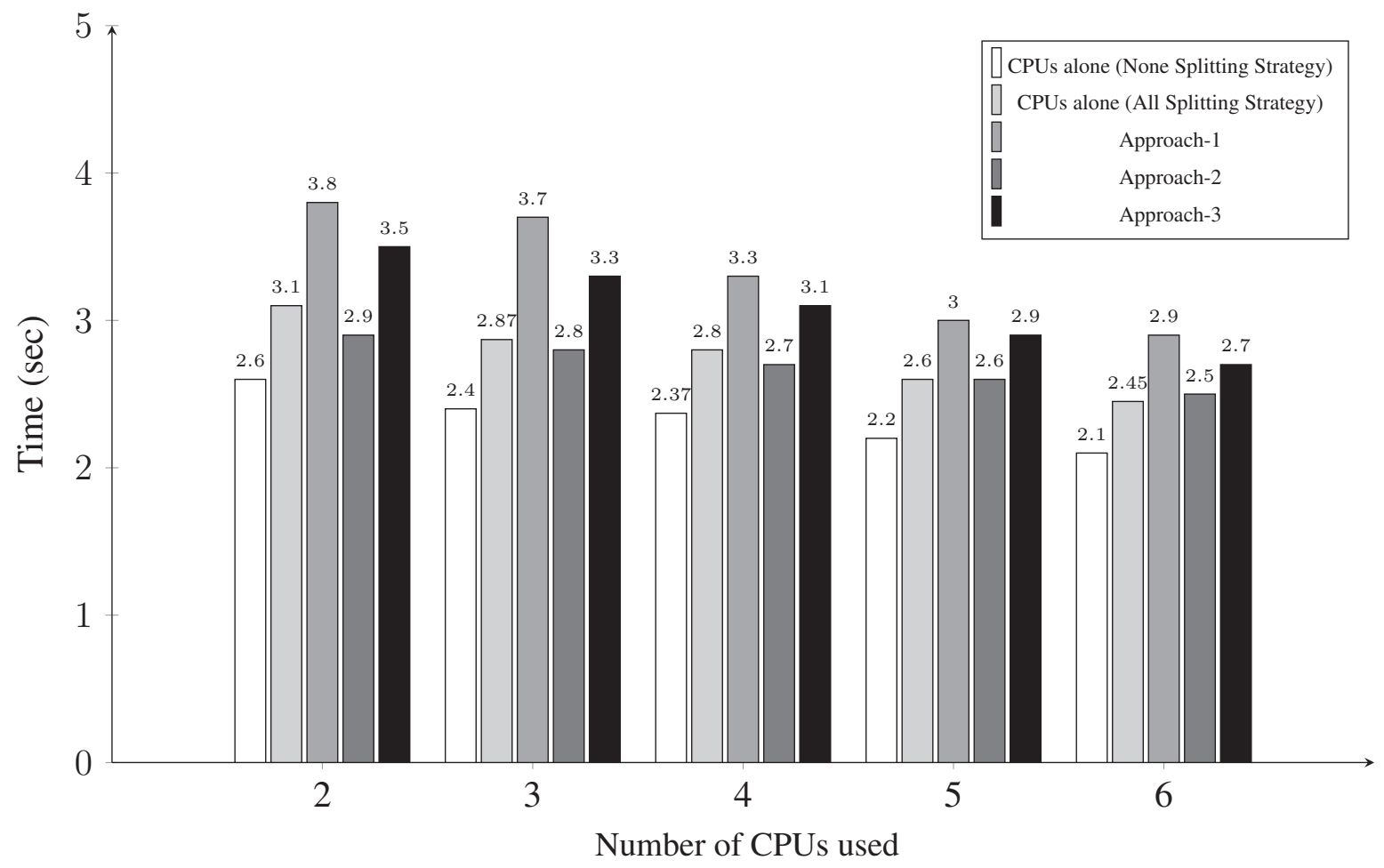

Figure 6.1: Performance Comparison of Approach-1 with multiple CPUs and a single GPU, Approach-2 and Approach-3 versus the case of just the Multiple CPUs 
Here, $x$-axis represents the number of CPUs used and $y$-axis represents the time taken to execute a single PR iteration in VF. For this example, CPU based approaches perform better compared to GPU based approaches due to the small size of the $\boldsymbol{A}_{l}$ matrix. For a proper load balancing for Approach-2 and Approach-3, in this example $n_{g}$ and $n_{c}$ are selected as in Table 6.1

Figures 6.2 and 6.3 provides an accuracy comparison between the converged VF model using Approach-3 versus the original model for sample S-parameter elements. As seen, results from both approaches are in good agreement.

\begin{tabular}{|c|c|c|c|c|}
\hline \multicolumn{5}{|c|}{ For one $\mathrm{PR}$ iteration $\left(P^{2}=30 * 30=900\right.$ elements $)$} \\
\hline \multirow{2}{*}{$\begin{array}{l}\text { Number of } \\
\text { CPUs used } \\
\text { in parallel }\end{array}$} & \multicolumn{2}{|c|}{$\begin{array}{l}\text { Number of } A_{l} \text { elements sent to } \\
\text { GPU }\end{array}$} & \multicolumn{2}{|c|}{$\begin{array}{l}\text { Number of } A_{l} \text { elements sent to } \\
\text { CPUs }\end{array}$} \\
\hline & $n_{g}$ & $\frac{n_{g}}{P^{2}} \times 100$ & $n_{c}$ & $\frac{n_{C}}{P^{2}} \times 100$ \\
\hline 2 & 423 & $47 \%$ & 477 & $53 \%$ \\
\hline 3 & 333 & $37 \%$ & 567 & $63 \%$ \\
\hline 4 & 198 & $22 \%$ & 702 & $78 \%$ \\
\hline 5 & 135 & $15 \%$ & 765 & $85 \%$ \\
\hline 6 & 108 & $12 \%$ & 792 & $88 \%$ \\
\hline
\end{tabular}

Table 6.1: $n_{g}$ and $n_{c}$ values for Example 1 for multiport VF 

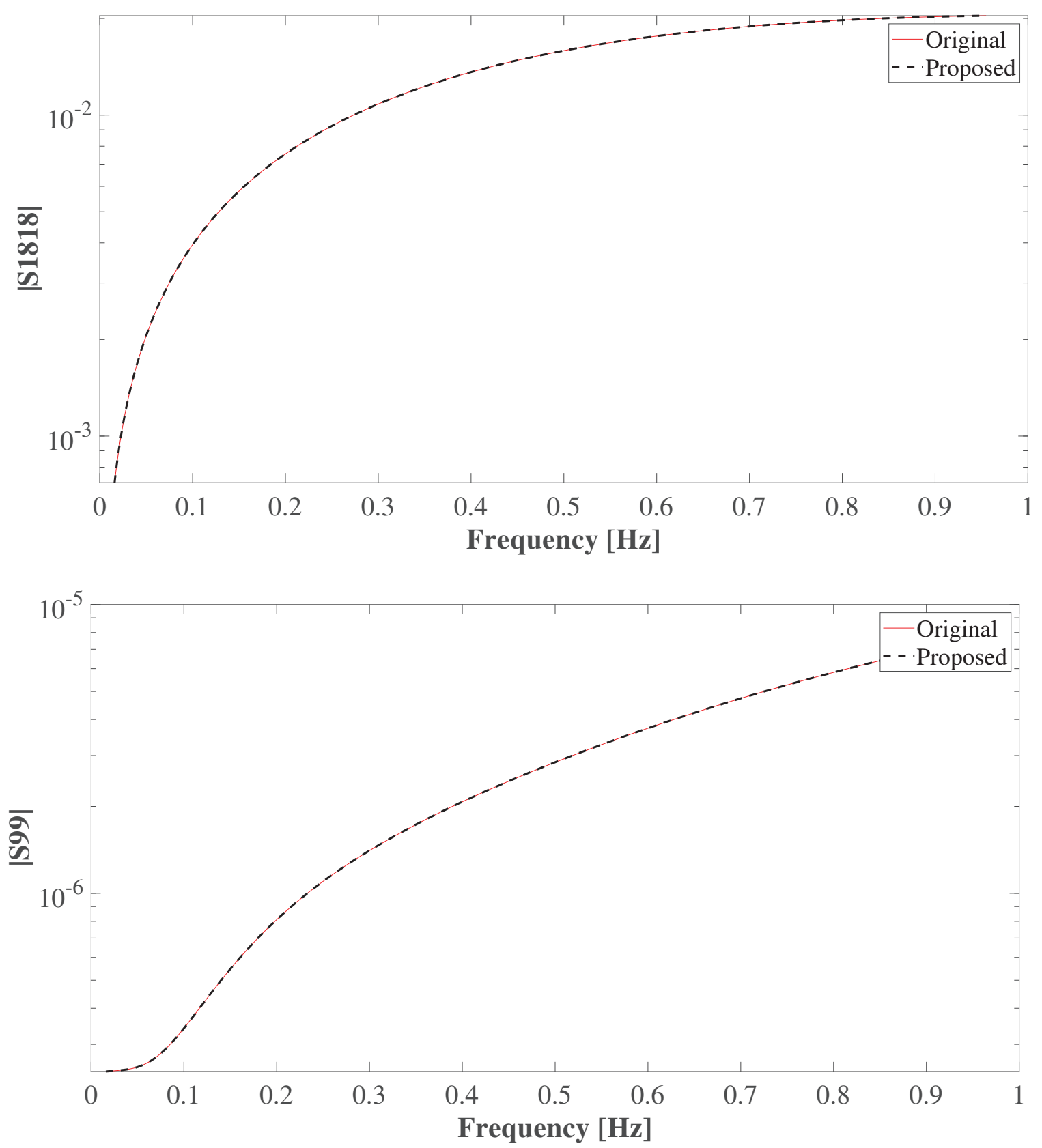

Figure 6.2: Comparison of sample frequency responses for Example 1 

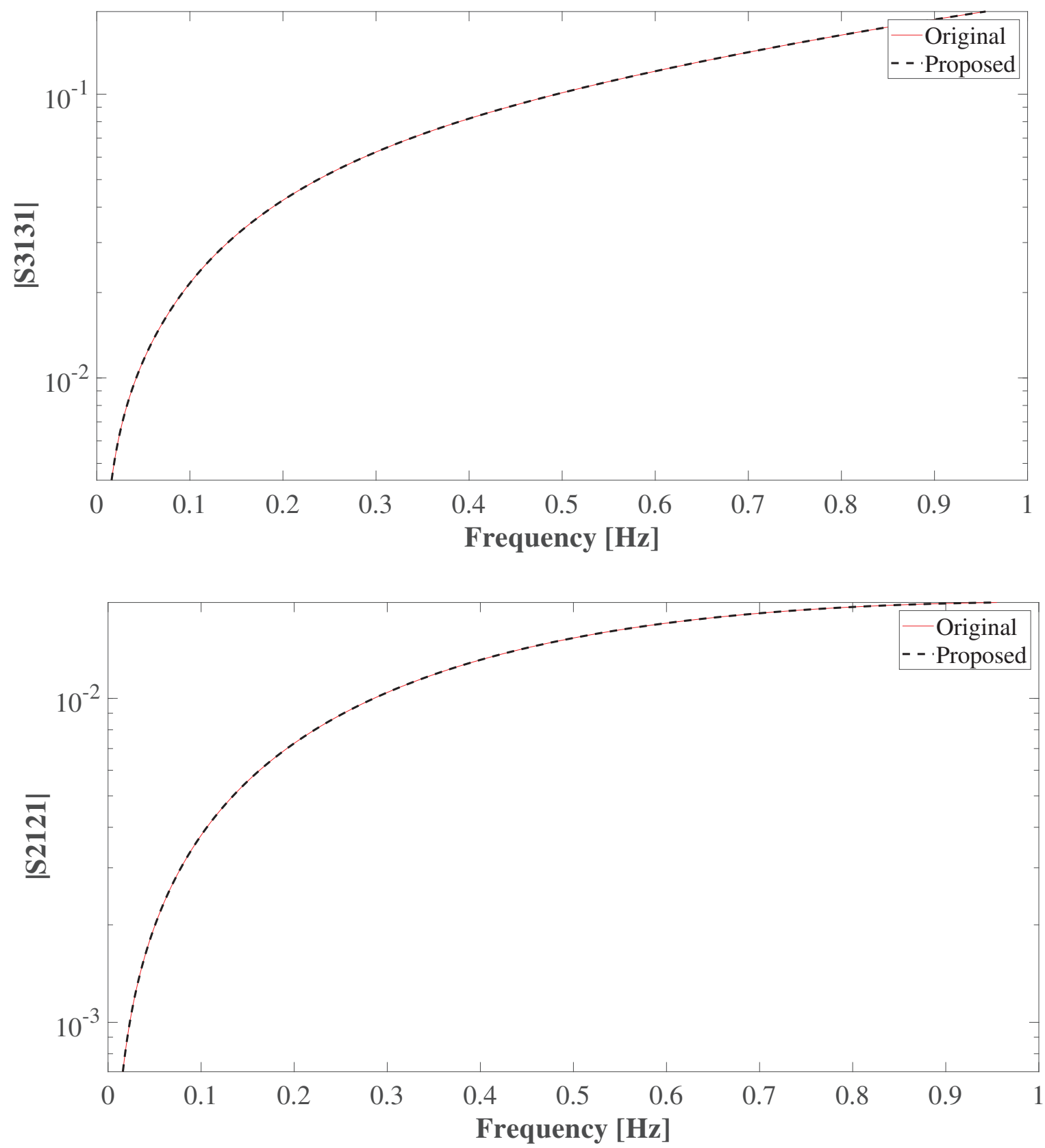

Figure 6.3: Comparison of sample frequency responses for Example 1 


\subsection{Example 2 : 60 port network}

In this example, the performance comparison of Approach-1 (multiple CPUs and a single GPU), Approach-2 and Approach-3 versus the case of just the multiple CPUs is given for a system with 60 port $(P), 70$ poles $(N)(35$ complex poles, 35 complex conjugate poles) and 1000 frequency samples $(K)$. Note that corresponding size of each $\boldsymbol{A}_{l}$ matrix is $(2000 \times 142)$.

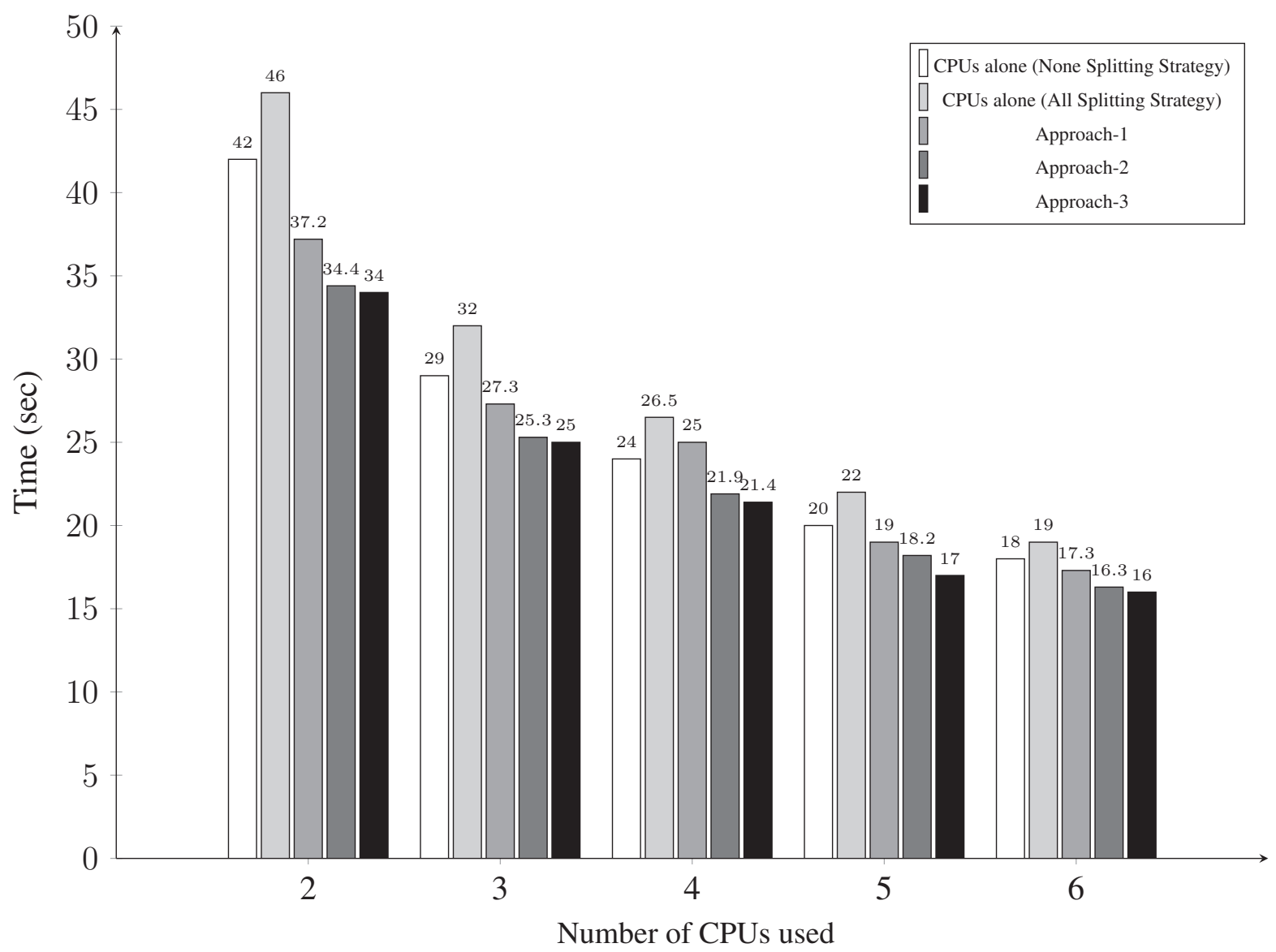

Figure 6.4: Performance Comparison of Approach-1 with multiple CPUs and a single GPU, Approach-2 and Approach-3 versus the case of just the Multiple CPUs 
Here, $x$-axis represents the number of CPUs used and $y$-axis represents the time taken to execute a single PR iteration in VF. It is observed that GPU based approaches provide either better or comparable performance with that of using just the multiple CPUs. Also with the use of more CPUs, parallelization increases, leading to less computational time. For a proper load balancing for Approach-2 and Approach-3, in this example $n_{g}$ and $n_{c}$ are selected as in Table 5.2

Figures 6.5 and 6.6 provide an accuracy comparison between the converged VF using Approach-3 versus the original data for sample S-parameter elements. As seen, results from both the approaches are in good agreement. 

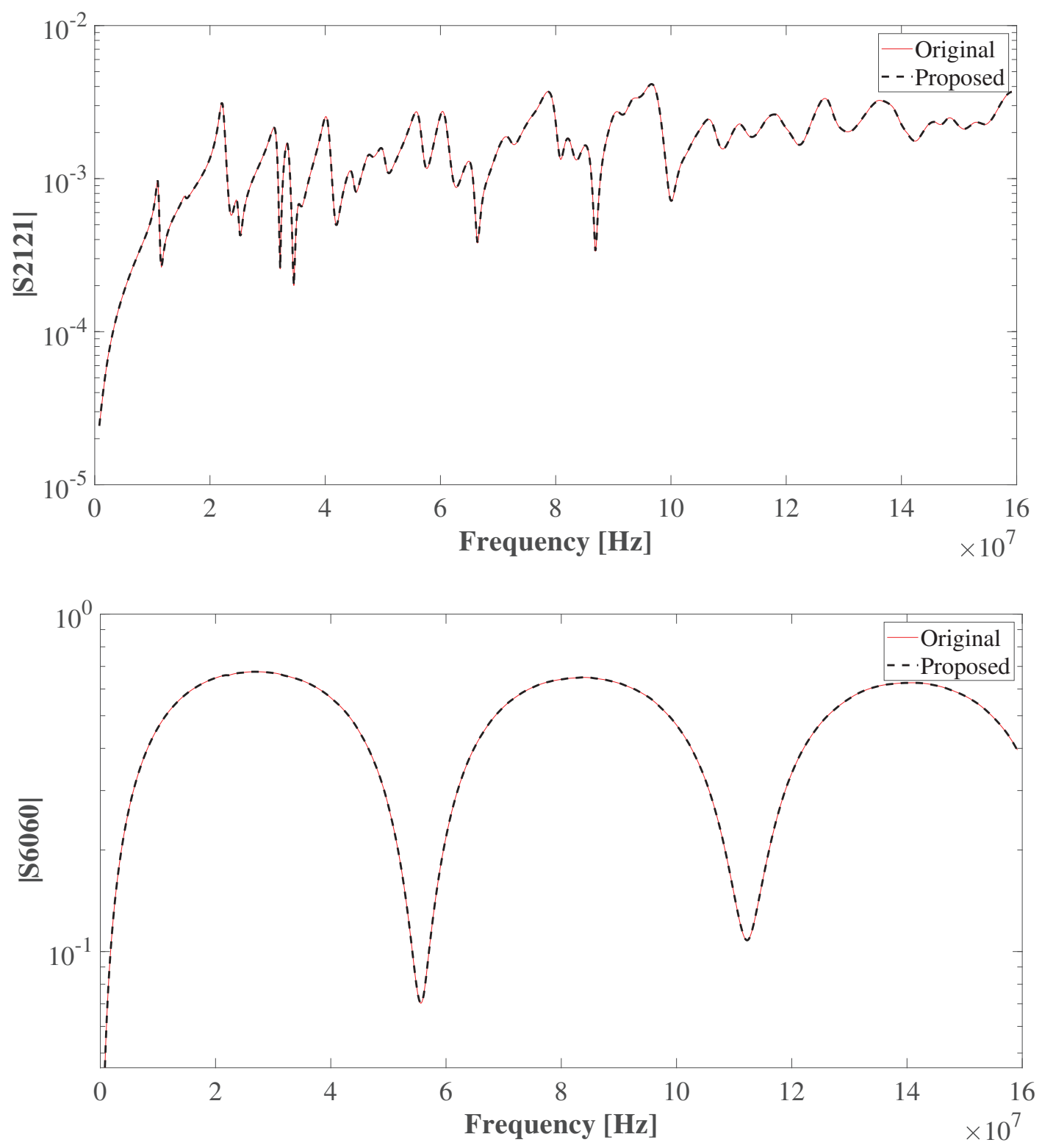

Figure 6.5: Comparison of sample frequency responses for Example 2 

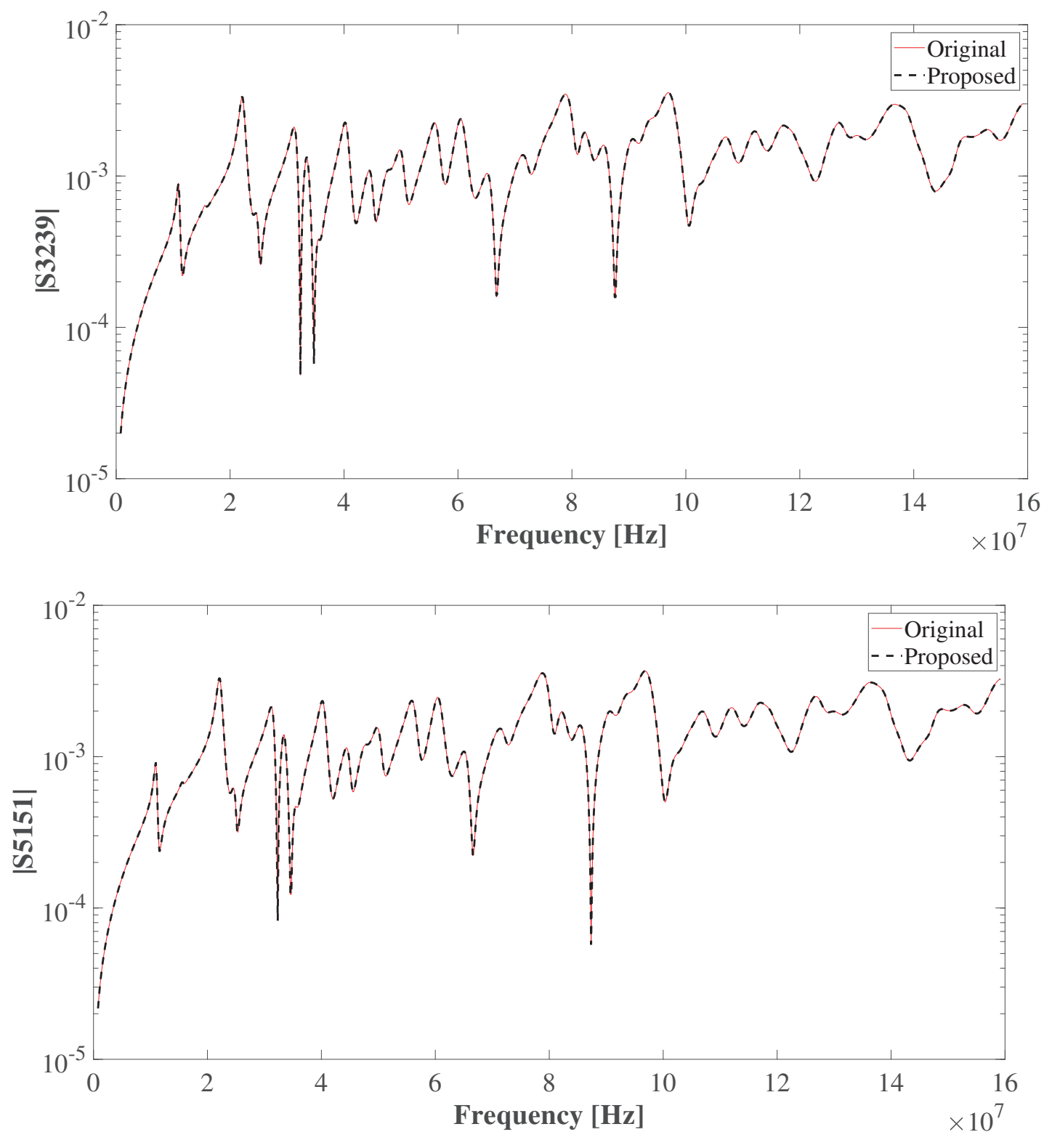

Figure 6.6: Comparison of sample frequency responses for Example 2 


\subsection{Example 3 : 120 port network}

In this example, the performance comparison of Approach-1 (multiple CPUs and a single GPU), Approach-2 and Approach-3 versus the case of just the multiple CPUs is given for a system with 120 port $(P), 80$ poles $(N)(40$ complex poles, 40 complex conjugate poles) and 1000 frequency samples $(K)$. Note that corresponding size of each $\boldsymbol{A}_{l}$ matrix is $(2000 \times 162)$.

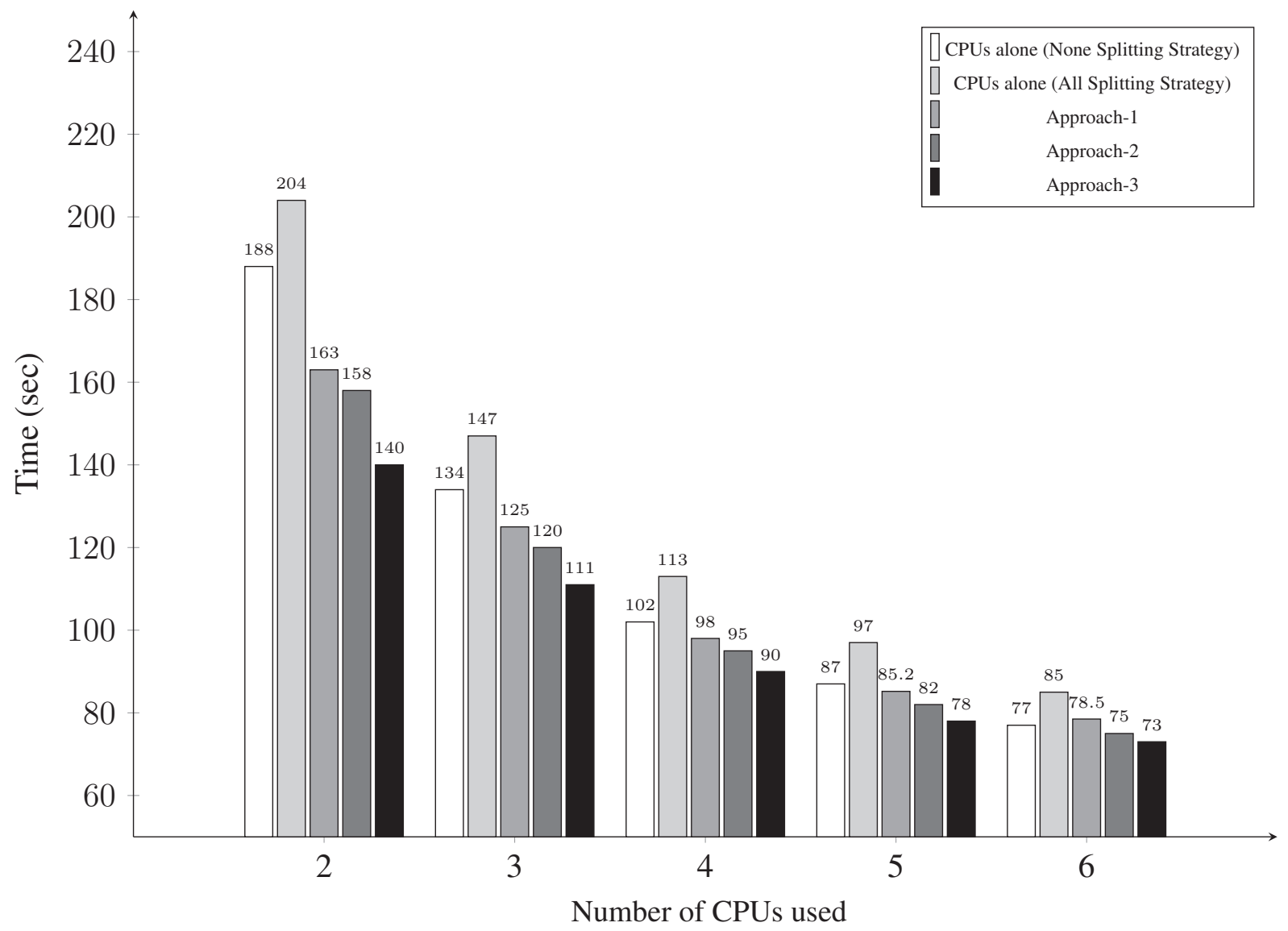

Figure 6.7: Performance Comparison of Approach-1 with multiple CPUs and a single GPU, Approach-2 and Approach-3 versus the case of just the Multiple CPUs 
Here, $x$-axis represents the number of CPUs used and $y$-axis represents the time taken to execute a single PR iteration in VF. It is observed that GPU based approaches provide either better performance with that of using just the multiple CPUs. Also with the use of more CPUs, parallelization increases, leading to less computational time. For a proper load balancing for Approach-2 and Approach-3, in this example $n_{g}$ and $n_{c}$ are selected as in Table 6.2

Figures 6.8 and 6.9 provide an accuracy comparison between the converged VF using Approach-3 versus the original data for this example for sample of S-parameter elements. As seen, results from both the approaches are in good agreement.

\begin{tabular}{|l|l|l|l|l|}
\hline \multicolumn{6}{|c|}{ For one PR iteration $\left(\boldsymbol{P}^{\mathbf{2}}=120 * 120=14400\right.$ elements $)$} \\
\hline $\begin{array}{l}\text { Number of } \\
\begin{array}{l}\text { CPUs used } \\
\text { in parallel }\end{array}\end{array}$ & $\begin{array}{l}\text { Number of } \boldsymbol{A}_{\boldsymbol{l}} \text { elements sent to } \\
\text { GPU }\end{array}$ & $\begin{array}{l}\text { Number of } \boldsymbol{A}_{\boldsymbol{l}} \text { elements sent to } \\
\text { CPUs }\end{array}$ \\
\cline { 2 - 5 } & \multicolumn{2}{|c|}{$\boldsymbol{n}_{\boldsymbol{g}}$} & $\frac{\boldsymbol{n}_{\boldsymbol{g}}}{\boldsymbol{P}^{\mathbf{2}}} \mathbf{X} \mathbf{1 0 0}$ & \multicolumn{2}{c|}{$\boldsymbol{n}_{\boldsymbol{c}}$} & $\frac{\boldsymbol{n}_{\boldsymbol{C}}}{\boldsymbol{P}^{\mathbf{2}}} \mathbf{X} \mathbf{1 0 0}$ \\
\hline 2 & 10512 & $73 \%$ & 3888 & $27 \%$ \\
\hline 3 & 8208 & $57 \%$ & 6192 & $43 \%$ \\
\hline 4 & 6624 & $46 \%$ & 7776 & $54 \%$ \\
\hline 5 & 5472 & $38 \%$ & 8928 & $62 \%$ \\
\hline 6 & 4752 & $33 \%$ & 9648 & $67 \%$ \\
\hline
\end{tabular}

Table 6.2: $n_{g}$ and $n_{c}$ values for Example 3 for multiport VF 

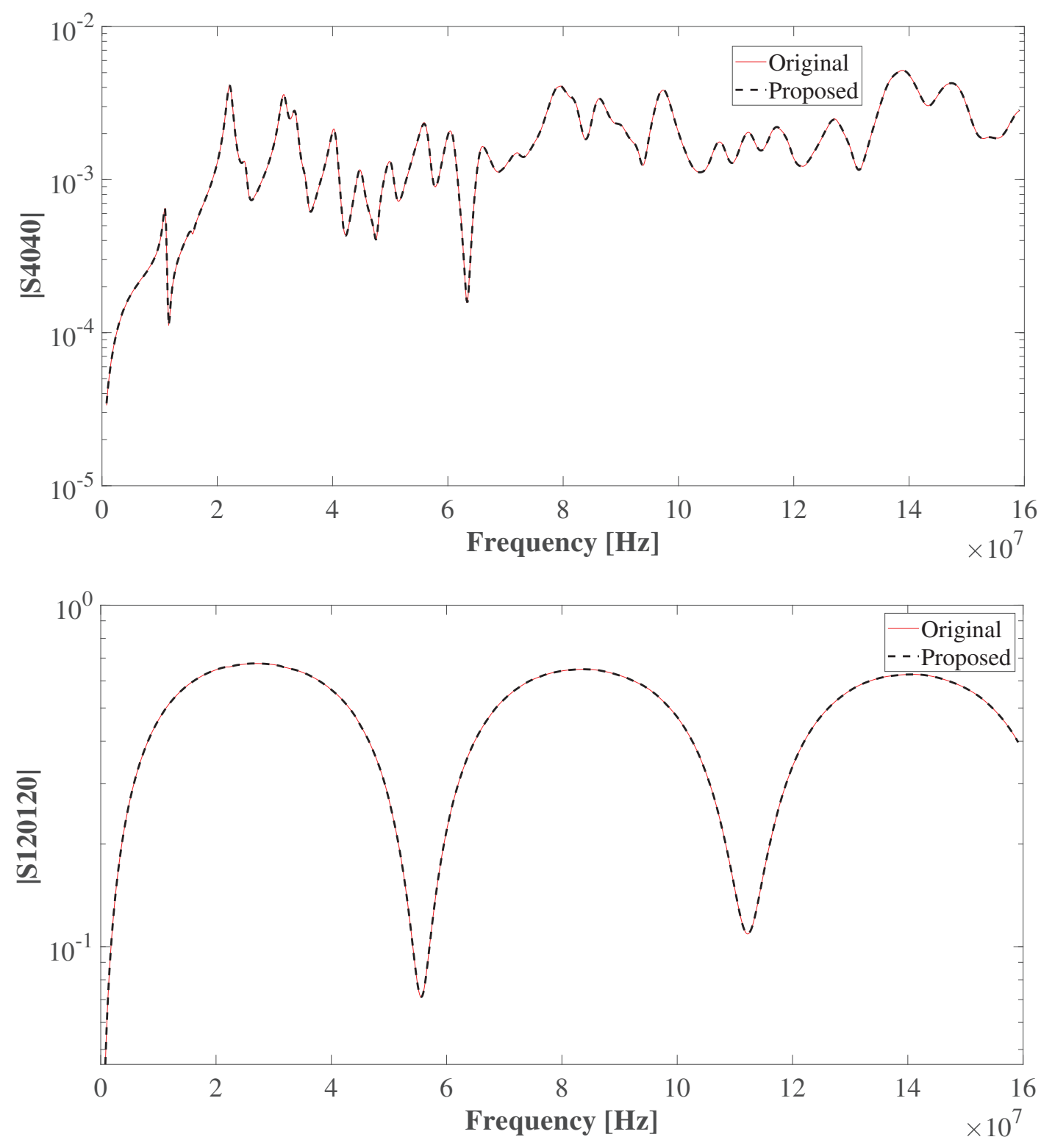

Figure 6.8: Comparison of sample frequency responses for Example 3 

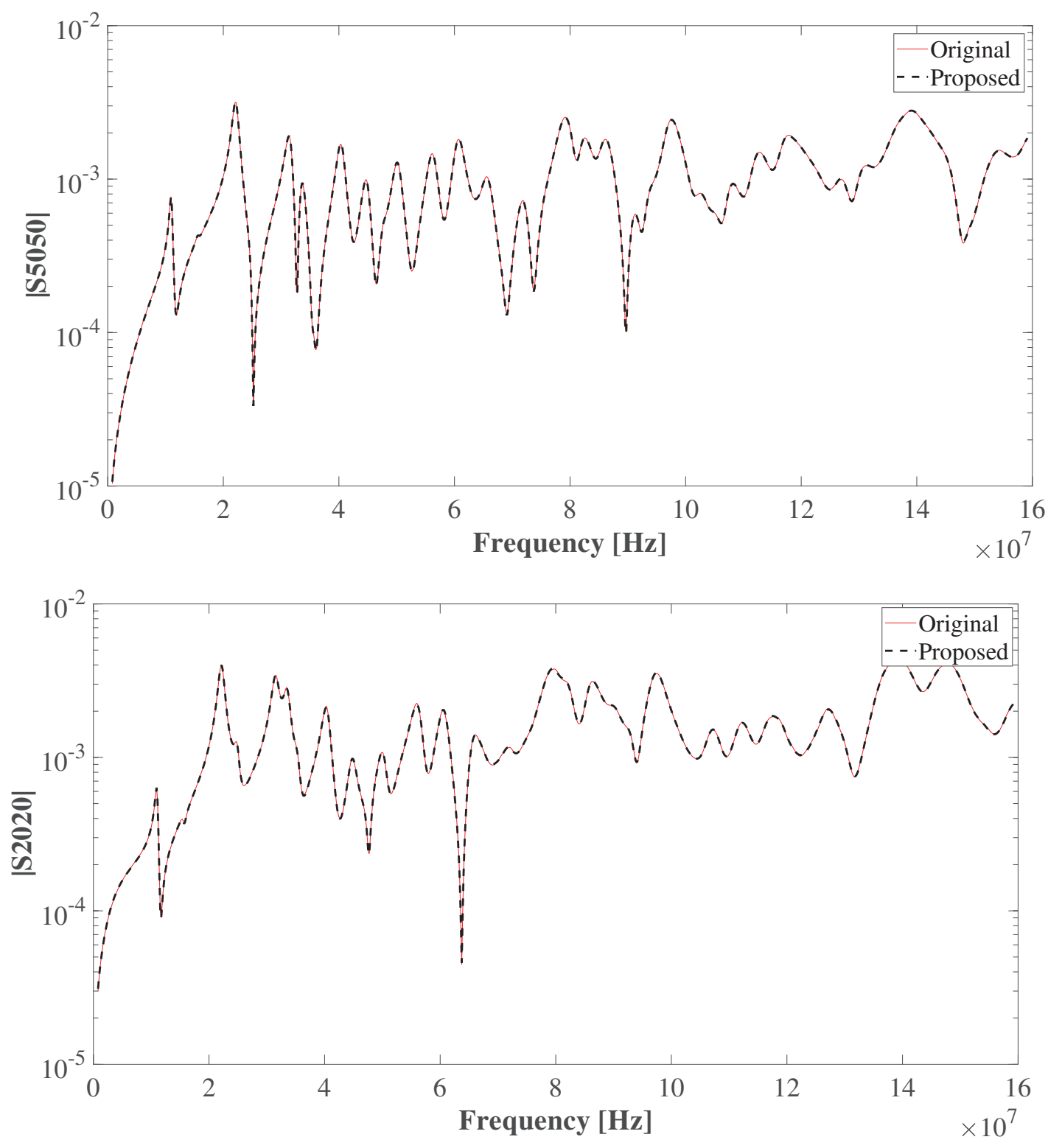

Figure 6.9: Comparison of sample frequency responses for Example 3 


\section{Chapter 7}

\section{Conclusion}

This thesis introduced several GPU-based parallelization approaches for VF. These include Approach-1, where the None Splitting Strategy was explored with QR factorization done sequentially in GPU while other tasks were handled by the CPUs. In Approach-2, instead of sequential QR factorization in GPU, parallel QR factorization for a block of $\boldsymbol{A}_{l}$ elements in GPU was explored while CPUs were also kept engaged with the $\mathrm{QR}$ factorization of rest of the $\boldsymbol{A}_{l}$ elements. In Approach-3, not only the parallel QR factorization for a block of $\boldsymbol{A}_{l}$ elements but also subsequent calculation of residues of the individual scaling elements in GPU was proposed.

As seen from the performance results, for small size $\boldsymbol{A}_{l}$ elements, QR factorization in CPU is much faster than in GPU and hence GPU based approaches do not yield better performance. However, as the $\boldsymbol{A}_{l}$ matrix size grows (as in the cases of large number ports, poles and frequency points), GPU based algorithms, particularly Approach-3, yield better performance compared to the case of using just the CPUs. 


\section{References}

[1] J. E. Schutt-Aaine and R. Mittra, "Scattering parameter transient analysis of transmission lines loaded with nonlinear terminations," IEEE Trans. Microwave Theory Tech., vol. 36, pp. 529-536, 1988.

[2] A. R. Djordjevic and T. K. Sarkar, "Analysis of lossy transmission lines with arbitary nonlinear terminal networks", IEEE Trans. Microwave Theory and Techniques, vol. 34, no. 6, pp. 660-666, June 1986.

[3] D. Winklestein, M. B. Steer, and R. Pomeerleau, "Simulation of arbitrary transmission line networks with nonlinear terminations," IEEE Transactions on Circuit Systems, vol. 38, pp. 418-422, April 1991.

[4] HSPICE Applications Manual [Online].

[5] R. Achar and M. Nakla, "Efficient transient Simulation of embedded subnetworks characterized by S-parameters in the presence of nonlinear elements," IEEE Trans. Microwave Theory and Techniques, vol. MTT-46, pp. 2356-2363, Dec. 1998.

[6] W. T. Beyene and J. E. Schutt-Aine, "Efficient Transient Simulation of HighSpeed Interconnects Characterized by Sampled Data," IEEE Transactions on Components, Packaging, and Manufacturing Technology, Part B, vol. 21, no. 1, pp. 105-114, Feb, 1998. 
[7] W. T. Beyene and J. E. Schutt-Aine, "Interconnect Simulation Using Order Reduction and Scattering Parameters," IEEE Electronic Components and Technology Conference, pp. 627-631, 1998.

[8] M. Elzinga, K. L. Virga and J. L. Prince, "Improved Global Rational Approximation Macromodeling Algorithm for Networks Characterized by FrequencySampled Data," IEEE Trans. Microwave Theory and Techniques, vol. 48, no. 9, Sept. 2000.

[9] W. T. Beyene, and J. E. Schutt-Aaine, "Accurate frequency-domain modelling and simulation of high-speed packaging interconnects", IEEE Trans. Microwave Theory Tech., pp. 1941-1947, Oct. 1997.

[10] B. Gustavsen and A. Semlyen, "Rational Approximation of Frequency Domain Responses by Vector Fitting," IEEE Transactions on Power Delivery, vol. 14, no. 3, pp. 1052-1061, July 1999.

[11] L. M. Silveria, I. M. Elfadel, J. K. White, M. Chilukuri and K. S. Kundert, "An efficient approach to transmission line simulation using measured or tabulated S-parameter data," in Proc. ACM/IEEE Electronic Components and Technology Conference, pp. 634-639, June 1994.

[12] M. Nakhla, E. Chiprout, R.Achar and R. Khazaka,"Recent Progress in simulation of high speed interconnects using moment matching techniques", Proc. IEEE 4th Topical Meeting on Electrical Performance of Electronic Packaging, Oct 1995 (Portland, Oregon, USA) 
[13] M. Nakhla, R. Khazaka, R. Achar, " A universal macromodeling technique for high-speed VLSI interconnects", 1st IEEE International Workshop on Signal Propagation on Interconnects, Hannover, Germany, May 1997.

[14] M. Elzinga, K. L. Virga and J. L. Prince, "Pole-residue formulation for transient simulation of high-frequency interconnects using householder LS curvefitting techniques," IEEE Trans. Advanced Packaging, vol. 25, pp. 142-147, May. 2000.

[15] G. H. Golub and C. F. Van Loan, Matrix Computations, 3rd edition, Baltimore, MD: Johns Hopkins Univ. Press, 1996.

[16] Alessandro Chinea and Stefano Grivet-Talocia, "On the Parallelization of Vector Fitting Algorithms", IEEE Trans. on Components, Packaging and Manufacturing Technology, vol. 1, no. 11, November 2011.

[17] K. H. Jin, M. T. McCann, E. Froustey, and M. Unser, "Deep convolutional neural network for inverse problems in imaging," IEEE Trans. Image Process., vol. 26, no. 9, pp. 4509-4522, Sep. 2017.

[18] X.-X. Liu, H. Yu, and S. X.-D. Tan, "A GPU-accelerated parallel shooting algorithm for analysis of radio frequency and microwave integrated circuits," IEEE Trans. Very Large Scale Integr. (VLSI) Syst., vol. 23, no. 3, pp. 480-492, Mar. 2015.

[19] B. D. de Vos, J. M. Wolterink, P. A. de Jong, T. Leiner, M. A. Viergever, and I. Isgum, "ConvNet-based localization of anatomical structures in 3-D medical images," IEEE Trans. Med. Imag., vol. 36, no. 7, pp. 1470-1481, Jul. 2017. 
[20] cuBLAS LIBRARY Users's Guide (2019, March) [Online]. Available: https://docs.nvidia.com/cuda/cublas/index.html

[21] B. Gustavsen, "Improving the pole relocating properties of vector fitting," IEEE Trans. Power Delivery, vol. 21, no. 3, pp. 1587-1592, Aug. 2006.

[22] A. C. Antoulas, Approximation of Large-Scale Dynamical Systems. Philadelphia, PA: SIAM, 2005.

[23] B. Gustavsen, "Relaxed Vector Fitting Algorithm for Rational Approximation of Frequency Domain Responses," 2006 IEEE Workshop on Signal Propagation on Interconnects, May 2006.

[24] Compute Canada. Accessed: April. 2019. [Online]. Available: https://www.computecanada.ca/home/

[25] Dirk Deschrijver, Michal Mrozowski, Tom Dhaene and Daniel De Zutter, "Macromodeling of Multiport Systems Using a Fast Implementation of the Vector Fitting Method," IEEE Microwave and Wireless Components Letters vol. 18, no. 6, June 2008. 